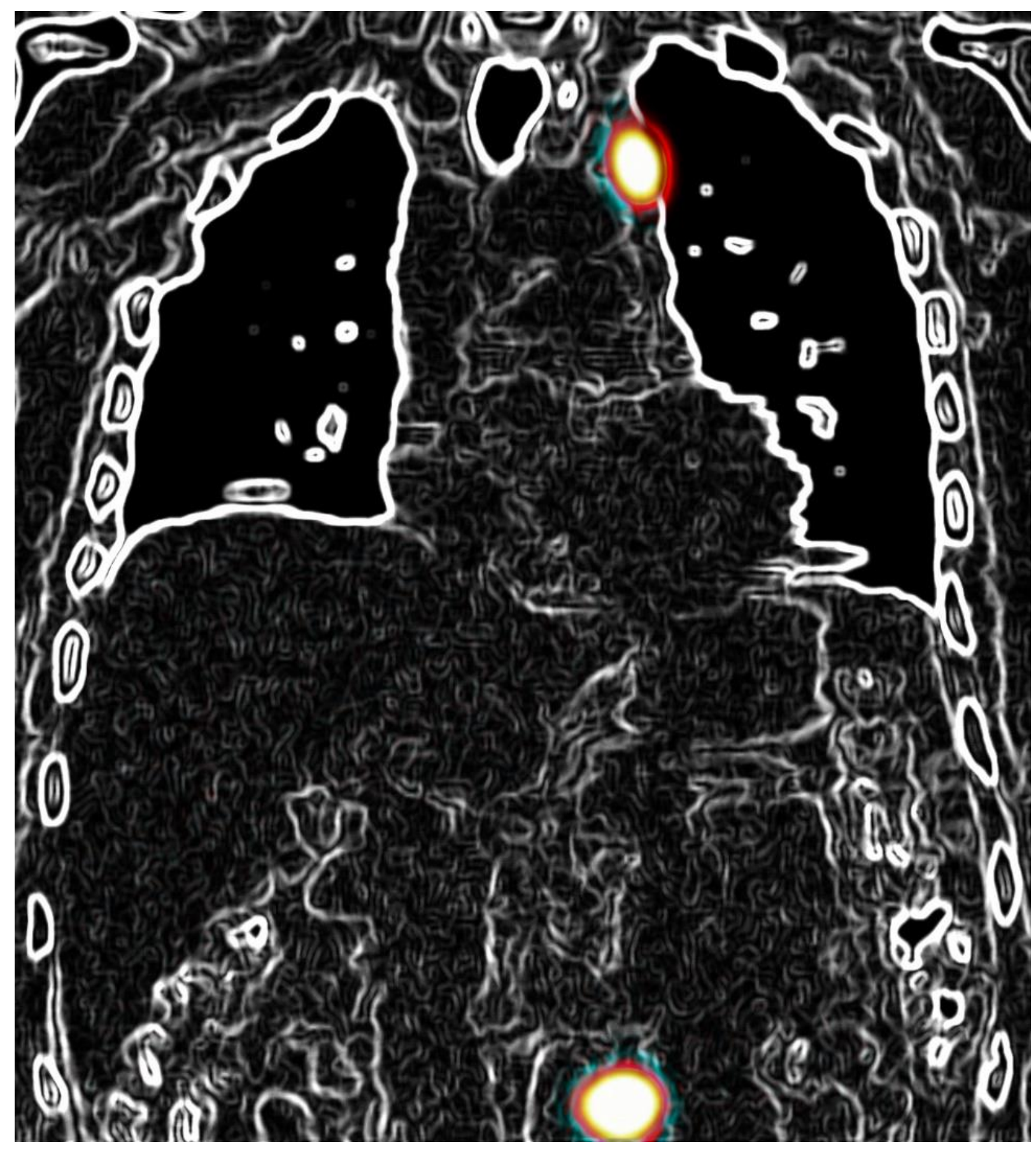

LYMPHATIC DRAINAGE IN RENAL

TUMOURS AND IMPLIC ATIONS FOR MANAGEMENT

TEELE KUUSK 
Financial support for printing this thesis was provided by: Airi Poder

Cover design by: Maria Meos

\section{(C) 2020 Teele Kuusk}

All rights reserved. No part of this thesis may be reproduced or transmitted, in any forms or by any means, without permission of the author. 


\title{
Lymphatic drainage in renal tumours and implications for management
}

\author{
Lymfedrainage bij niertumoren en implicaties voor beleid \\ (met een samenvatting in het Nederlands)
}

\section{Proefschrift}

Ter verkrijging van de graad van Doctor aan de Univeristeit Utrecht op gezag van de rector Magnificus, Prof.Dr.

H.R.B,M. Kummeling,

Ingevolge het besluit van het college voor promoties in het openbaar te verdedigen op donderdag 19 november 2020 des middags te 2.30 uur

door

Teele Kuusk

geboren op 12 juni 1984 te Tartu, Estland 
Promotor: Prof. Dr. S. Horenblas

Copromotor: Prof. Dr. A. Bex

Leden Promotiecommissie: Prof. Dr. H. P. Beerlage

Prof. Dr. R. de Bree

Prof. Dr. L.M.O. de Kort

Prof. Dr. M. G.E.H. Lam

Prof. Dr. F.W.B van Leeuwen

Prof. Dr. P.F.A. Mulders 
To my Family, Godsons, Mentors, Urology community and Patients 


\section{Contents}

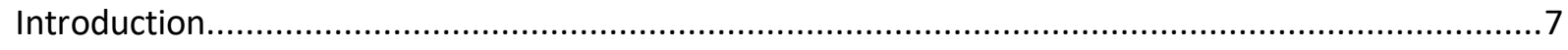

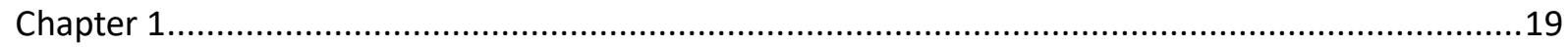

Antiangiogenic therapy combined with immune checkpoint blockade in renal cancer ..................19

CHAPTER 2

Lymphatic drainage from renal tumors in vivo: a prospective sentinel node study ........................42

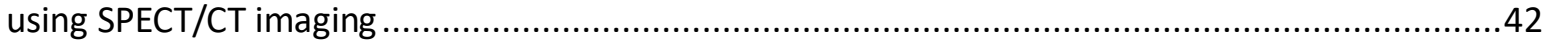

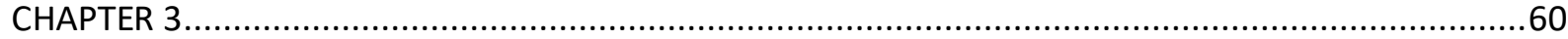

Outcome of sentinel lymph node biopsy in patients with clinically non-metastatic renal cell

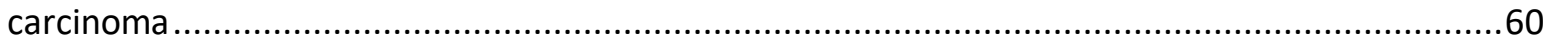

CHAPTER 4

Long-term Survival After Resection of Sentinel Node Metastatic Renal Cell Carcinoma. ................80

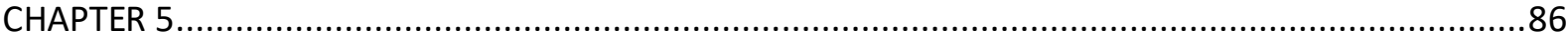

Topographic distribution of first landing sites of lymphatic metastases from patients with renal

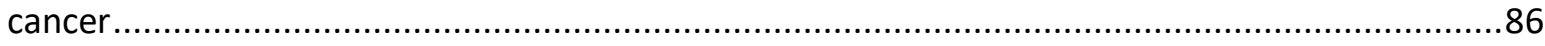

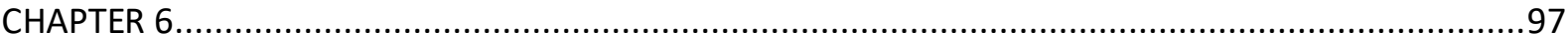

An analysis of SPECT/CT non-visualization of sentinel lymph nodes in renal tumors .....................97

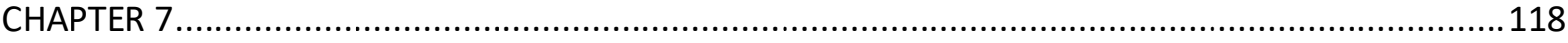

Sentinel Lymph Node Biopsy in Renal Tumors: Surgical Technique and Safety...........................118

CHAPTER 8

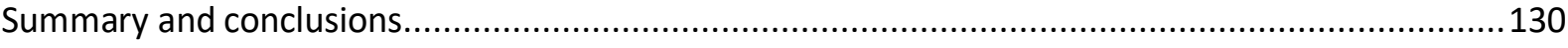

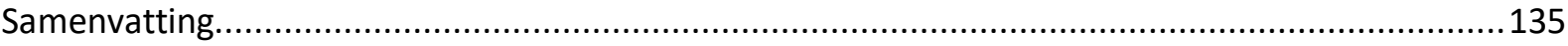

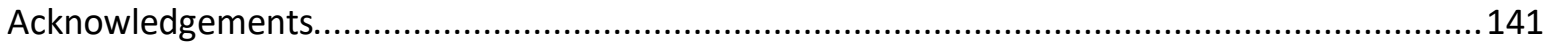

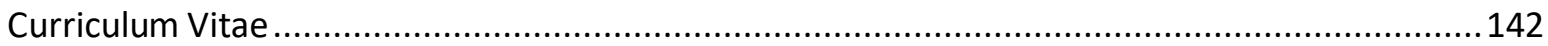

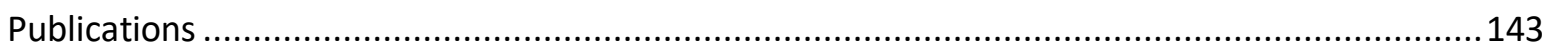




\section{Introduction}

\section{Renal Cell Carcinoma epidemiology}

Kidney cancer is the ninth and fourteenth most common cancer in men and women, representing $5 \%$ and $3 \%$ of all malignancies, respectively $[1,2]$. It is one of the most lethal urological cancers with a mortality rate between $30 \%$ and $40 \%$ [3]. However, mortality trends have been stable in most of the countries showing even a decrease in Western and Northern European countries [1]. Worldwide, there are approximately 403, 262 new cases diagnosed yearly and 175, 098 deaths due to kidney cancer [4]. Renal cell carcinoma (RCC) represents $90 \%$ of kidney cancers, with clear cell (70-80\%), papillary (10-15\%), and chromophobe (5\%) being the main histological subtypes [5]. RCC incidence varies globally between 1 and 22/100,000, being highest in the elderly population (>75 years old) [1]. During the last decade, incidence has been increasing in most countries, although a trend towards plateauing or even decreasing has been seen in developed countries [1].

\section{Diagnosis and management}

Many renal masses are asymptomatic and have been diagnosed in the past only in advanced stages. The classic triad of a palpaple mass, flank pain and hematuria is now rare as most renal tumours are currently diagnosed incidentally by cross sectional Imaging or ultrasound. Especially computed tomography (CT) or magnetic resonance imaging (MRI) of the abdomen and pelvis are used to characterise renal masses and to assess extension of the primary tumour, venous involvement, enlargement of locoregional lymph nodes and involvement of adrenal glands or other solid organs. A CT Chest completes the staging for which the Tumour Node Metastasis (TNM) classification system is recommended. The most recent version is the 2017 TNM classification (table 1) [6].

Table 1: TNM classification, $8^{\text {th }}$ edition

\begin{tabular}{|ll|}
\hline T - Primary Tumour \\
\hline TX & Primary tumour cannot be assessed \\
T0 & No evidence of primary tumour \\
T1 & Tumour $\leq 7 \mathrm{~cm}$ or less in greatest dimension, limited to the kidney \\
T1a & Tumour $\leq 4 \mathrm{~cm}$ or less \\
T1b & Tumour $>4 \mathrm{~cm}$ but $\leq 7 \mathrm{~cm}$ \\
T2 & Tumour $>7 \mathrm{~cm}$ in greatest dimension, limited to the kidney \\
T2a & Tumour $>7 \mathrm{~cm}$ but $\leq 10 \mathrm{~cm}$ \\
T2b & Tumours $>10 \mathrm{~cm}$, limited to the kidney \\
\hline
\end{tabular}


T3 Tumour extends into major veins or perinephric tissues but not into the ipsilateral adrenal gland and not beyond Gerota fascia

T3a Tumour grossly extends into the renal vein or its segmental (muscle-containing) branches, or tumour invades perirenal and/or renal sinus fat (peripelvic fat), but not beyond Gerota fascia

T3b Tumour grossly extends into vena cava below diaphragm

T3c Tumour grossly extends into vena cava above the diaphragm or invades the wall of the vena cava

T4 Tumour invades beyond Gerota fascia (including contiguous extension into the ipsilateral adrenal gland)

\section{N - Regional Lymph Nodes}

NX Regional lymph nodes cannot be assessed

NO No regional lymph node metastasis

N1 Metastasis in regional lymph node(s)

\section{M - Distant Metastasis}

M0 No distant metastasis

M1 Distant metastasis

Despite earlier detection rates, $25-30 \%$ of renal cell carcinomas are metastatic at diagnosis [7]. Recent figures suggest that this rate has declined to $<20 \%[8,9]$. In addition, approximately $30 \%$ of all patients with renal cell carcinoma develop metastases after local therapy with curative intent for clinically non-metastatic disease and its heterogenic biology may impact on the pattern and frequency of metastasis which differ from other genitourinary cancers [5]. Validated risk scores revealed that this rate is risk dependent [3] and mortality in metastatic disease is associated with metastatic sites [7]. Patients with distant metastasis at the time of diagnosis (primary or synchronous metastatic renal cell carcinoma [ $\mathrm{mRCC}$ ) ) or after curative intent (metachronous $\mathrm{mRCC}$ ) are currently recommended to undergo prognosis assessment according to validated prognostic scores [8]. Those with intermediate or poor prognosis or abscence of low-volume metastatic disease should be treated with systemic therapy [8]. The standard-of-care for treatment-naive patients with clear cell mRCC consists of combination immune checkpoint inhibitor therapy with either a combination of nivolumab, a monoclonal antibody against programmed death-1 (PD-1) and ipilimumab, a monoclonal antibody against cytotoxic T-lymphocyte associated protein 4 (CTLA-4), or, pembrolizumab, a monoclonal antibody against PD-1, and axitinib, a vascular endothelial growth factor receptor tyrosine kinase inhibitor [8]. Two recent randomized controlled trials investigating the role and timing of cytoreductive nephrectomy for patients with primary clear cell mRCC led to a paradigm change and upfront surgery is no longer recommended in this setting $[10,11]$.

However, in the absence of metastatic disease, surgery has developed by default as the benchmark for the treatment of renal tumours. Approach and technique depend on the size of the primary tumour and current management options for CT1a renal masses - also called small renal mass (SRM) 
- include nephron sparing strategies such as partial nephrectomy or thermal ablation, and active surveillance. Patients with SRM are increasingly offered renal mass biopsy for radiologically indeterminate renal lesions or before active surveillance of small masses. A recent analysis of 18.060 partial nephrectomies performed in the United States based on imaging alone revealed a $30 \%$ rate of benign tumours removed by surgery [12]. For small renal masses and cT1b tumours nephrectomy is no longer standard of care and partial nephrectomy should be offered when technically feasible and oncologically safe [8]. For larger tumours nephrectomy is the preferred treatment option performed as either minimally invasive laparoscopic or robotic assisted laparoscopic or open transperitoneal nephrectomy. The latter is often the preferred approach for locoregionally advanced disease including inferior vena cava (IVC) thrombi or clinically enlarged locoregional lymph nodes. Locally advanced disease itself is a risk factor for lymph node invasion in several nomograms, making predominantly use of the clinical $\mathrm{T}$ stage and clinical $\mathrm{N}$ stage [3].

\section{Synchronous lymph node metastases and management}

Even though it is believed that renal cancer spreads predominantly hematogenously, lymph nodes are the second to third most common metastatic site following lung or bone. Synchronous lymph node metastases are found in $41 \%$ and $12 \%$ of patients with multiple metastatic and solitary metastatic disease sites, respectively [7]. Survival with lymph node metastases is poor, with 5-year survival ranging between $20-30 \%[13,14,15]$ and for patients with resected isolated synchronous lymph node metastasis the median time to develop distant metastases has been reported to be only 4 months [16]. The extremely short time period for developing distant disease supports the hypothesis that lymph node positive patients may have concomitant occult systemic metastases accounting for the very poor prognosis. Data of these retrospective studies are difficult to interpret as most patients with pathologically confirmed lymph node metastases (pN1) underwent lymph node dissection (LND) because of clinically enlarged lymph nodes on imaging (CN1) and not as a routine procedure including patients with clinically node negative disease (cNO). Currently, LND does no longer belong to a routine procedure during partial or radical nephrectomy since guidelines do recommend LND only for patients with enlarged lymph nodes on imaging (cN1) but not for clinically node negative disease (cNO)[8]. This recommendation against routine LND is based on a single prospective study which showed no survival advantage with LND for clinically node negative renal cell carcinoma $[17,18]$. This study included patients with lower-risk clinically node-negative renal cancers and only $3.3 \%$ of the patients in the LND arm had lymph node metastases. This low lymph node metastasis rate significantly impacts the interpretation of the results of the study which was depending on an event-driven sample size calculation. In addition, no standardized templates were used. Some observational, retrospective studies suggest a better survival in a subgroup of patients with good prognostic features and with increased numbers of removed lymph nodes $[16,19,20]$. Especially after resection of isolated lymph node metastases in patients without adverse features survival reached 10 years [16]. However, as the randomized controlled trial on LND and other retrospective studies have shown, the rate of occult lymph node metastases in patients with cNO disease is very low, even in high risk RCC [21] and from these patients only a subgroup may have a favourable survival. Due to this low rate and inability of cross sectional imaging to reliably detect lymph node metastases in patients with clinically non-enlarged lymph nodes, the ability to identify and potentially cure patients with very limited occult lymph node metastases by LND is low. In 
addition, there are knowledge gaps regarding the biology of lymphatic spread and progression of renal cell carcinoma. Recent data suggest that renal cancer progression can occur according to punctuated, branched or linear evolution [22]. Patients with punctuated evolution of metastasis have multiple clonal driver mutations including von Hippel-Lindau (VHL), BRCA1-associated protein-1 (BAP1) and Su(var), Enhancer of zeste, Trithorax-domain containing 2 (SETD2) and present with rapid progression at multiple organ sites. On the contrary, those with branched or linear evolution have attenuated or very slow progression based on Polybromo 1 (PBRM1) or monoclonal VHL driver mutations. If and how this relates to lymph node metastases or why some patients develop predominantly lymphatic metastatic spread is unclear. Also, it is currently unknown, if the favourable outcome observed for patients after resection of limited occult lymph node metastases [18] is due to a less-aggressive tumour biology such as the observed branched or linear evolution, the LND performed, or a combination of both. Based on the available evidence, guidelines therefore do not recommend routine LND in cNO-disease [8], but it is regaining interest as a strong prognosticator in an era in which multiple adjuvant treatment trials have been performed or are ongoing due to more systemic therapeutic options [23]. Patients with pathologically confirmed lymph node metastatic RCC (pN1) have a higher risk of disease recurrence which is reflected in the fact that validated risk scores include pN1 as a significant risk factor. Several risk scores are in use and none has been compared head-to-head. Nevertheless, their accuracy is relatively high given that they are only based on clinical and pathological parameters (table 2). A simplified risk score, which is not yet externally validated, has been developed to stratify patients after LND for adjuvant trial enrollment [24] and the currently ongoing adjuvant trials include patients with pN1 disease selected by TNM staging criteria or Leibovich risk scoring.

Table 2: validated risk scores for non-metastatic RCC after surgical resection

\begin{tabular}{|c|c|c|c|c|}
\hline Risk model & Risk factors & subtype & Predictor & $\begin{array}{l}\text { Accuracy (c- } \\
\text { index) }\end{array}$ \\
\hline Leibovich $^{25}$ & $\begin{array}{l}\text { T-stage } \\
\text { Tumour size } \\
\text { Necrosis } \\
\text { LN status } \\
\text { Fuhrman grade }\end{array}$ & Clear-cell & DFS & 0.81 \\
\hline Leibovich updated $^{26}$ & $\begin{array}{l}\text { T-stage } \\
\text { Tumour size } \\
\text { Necrosis } \\
\text { LN status } \\
\text { Fuhrman grade }\end{array}$ & All subtypes & $\begin{array}{l}\text { PFS } \\
\text { CSS }\end{array}$ & $\begin{array}{l}\text { PFS: } \\
\text { ccRCC } 0.83 \text {, } \\
\text { papRCC } 0.77 \\
\text { chrRCC } 0.78 \\
\text { CSS: } \\
\text { ccRCC } 0.86 \\
\text { papRCC } 0.83\end{array}$ \\
\hline $\begin{array}{l}\text { Sorbellini nomogram } \\
27\end{array}$ & $\begin{array}{l}\text { T-stage } \\
\text { Tumour size } \\
\text { Necrosis } \\
\text { Vascular invasion } \\
\text { Fuhrman grade } \\
\text { Symptoms }\end{array}$ & Clear-cell & DFS & 0.82 \\
\hline Kattan nomogram ${ }^{28}$ & T-stage & All subtypes & DFS & 0.80 \\
\hline
\end{tabular}




\begin{tabular}{|l|l|l|l|l|}
\hline & $\begin{array}{l}\text { Tumour size } \\
\text { Histologic subtype }\end{array}$ & & $\begin{array}{l}\text { CSS } \\
\text { OS }\end{array}$ & 0.77 \\
& T- stage & Clear cell & DFS & 0.70 \\
\hline SSIGN & Tumour size & & & 0.81 \\
& $\begin{array}{l}\text { Cuhrman grade } \\
\text { Necrosis }\end{array}$ & & $0.83-0-88$ \\
\hline $\begin{array}{l}\text { UCLA-Integrated } \\
\text { Staging System (UISS) } \\
30\end{array}$ & $\begin{array}{l}\text { T-stage } \\
\text { N-stage } \\
\text { Fuhrman grade } \\
\text { ECOG PS }\end{array}$ & Clear cell & CSS & \\
\hline
\end{tabular}

Legend: LN lymph node, DFS disease-free survival, CSS cancer-specific survival, PFS progression-free survival, OS overall survival, ECOG PS Eastern Cooperative Oncology Group Performance Status, SSIGN The tumor stage, size, grade, and necrosis score, UCLA The University of California, Los Angeles

\section{Adjuvant therapy}

Despite multiple trials no adjuvant therapy for renal cell carcinoma is approved in Europe nor recommended by the European Association of Urology (EAU) guidelines. After the introduction of targeted therapy several trials have been performed of which only one has not yet reported. Details of the studies are presented in table 3. Results of these studies have not shown a statistically significant benefit in disease-free survival (DFS) with the exception of the S-TRAC trial, a multi-center double-blind placebo-controlled trial of 615 patients with high-risk recurrent renal cell carcinoma following nephrectomy. On November $16^{\text {th }} 2017$, the Food and Drug Administration (FDA) approved sunitinib for the adjuvant treatment of high-risk renal cancer patients with clear-cell subtype following nephrectomy. Given that other trials failed to detect a statistically significant benefit for adjuvant therapy, it has been postulated that the inclusion of a well-defined high-risk group with full dose sunitinib might have led to the S-TRAC trial demonstrating a significant DFS benefit. However, a post-hoc subset analysis from the ASSURE trial including the highest-risk patients and those starting with full dose sunitinib comparable to S-TRAC eligibility did not show any DFS or overall survival (OS) benefit [37]. Finally, the European Medicine Agency (EMA) did not approve sunitinib for adjuvant use in Europe based on a high grade 3-4 adverse event rate without a proven OS benefit [8]. Recent outcome with immunotherapy has revolutionized metastatic renal cancer treatment, increasing OS to a median of 28 months and more with combinations of ipilimumab/nivolumab and pembrolizumab/axitinib and hazard ratios of death of 0.66 and 0.53 when compared to the previous standard, sunitinib $[38,39]$. The success of immunotherapy has led to an interest to study these agents in the adjuvant setting. Based on the assumption, that immunotherapy is effective in eradication of micrometastatic disease, adjuvant immunotherapy is promising [40]. Most trials assess eligibility using the TNM or Leibovich risk classification which includes patients with resected lymph node metastases in the high risk groups. Consequently, LND, which has an unproven therapeutic advantage in renal cell carcinoma [17] and has not been performed routinely for decades, is regaining importance in high risk renal cancer for prognosis assessment in the adjuvant setting. Furthermore, if adjuvant studies with immunotherapy will demonstrate a DFS- or even OS-benefit, accurate prognostication with LND might regain relevance in treatment decision making and patient counselling. Currently, 5 phase III randomized controlled trials examine the effect of immunotherapy 
in the adjuvant setting for loco-regional intermediate to high-risk RCC: EA8143 PROSPER

[NCT03055013] $]^{41}$ investigates 4 weeks of neoadjuvant nivolumab followed by 1 year of nivolumab versus observation, IMmotion 010 [NCT03024996] $^{42}$ studies 1 year of atezolizumab versus placebo, KEYNOTE-564 [NCT03142334] ${ }^{43}$ investigates 15 months of pembrolizumab versus placebo, RAMPART [NCT03288532] $^{44}$, a multiarm designed trial platform, studies a combination of 1 year of durvalumab plus tremelimumab versus either 1 year of durvalumab alone or observation, and CheckMate 914 [NCT03138512] $]^{45}$ investigates 6 months of nivolumab plus ipilimumab versus placebo. While most trials are recruiting, two have completed accrual. The outcomes are not mature yet and are awaited in a few years time.

Table 3: Adjuvant trials in the era of targeted therapy

\begin{tabular}{|c|c|c|c|c|c|c|}
\hline Trial & $\mathbf{N}$ & $\begin{array}{l}\text { Patient } \\
\text { Characteristics }\end{array}$ & $\begin{array}{l}\text { Treatment } \\
\text { Arms }\end{array}$ & $\begin{array}{l}\text { Treatment } \\
\text { Duration }\end{array}$ & $\begin{array}{l}\text { Primary } \\
\text { End Point }\end{array}$ & \begin{tabular}{|l} 
Primary \\
end \\
point HR \\
p-value
\end{tabular} \\
\hline $\begin{array}{l}\text { S-TRAC: Sunitinib } \\
\text { Trial in Adjuvant } \\
\text { Renal Cancer } \\
\text { Treatment }^{31}\end{array}$ & 615 & $\begin{array}{l}\text { High-risk patients } \\
\text { according to UISS }\end{array}$ & $\begin{array}{l}\text { Sunitinib } \\
\text { Placebo }\end{array}$ & 1 year & DFS & $\begin{array}{l}6.8 \text { vs } \\
5.6 \text { years } \\
\text { HR } 0.76 \\
P=0.03\end{array}$ \\
\hline $\begin{array}{l}\text { ASSURE: Adjuvant } \\
\text { Sorafenib or } \\
\text { Sunitinib for } \\
\text { Unfavorable RCC } \text { R }^{32}\end{array}$ & 1,943 & $\begin{array}{l}\text { Non-metastatic RCC; } \\
\text { disease stage II-IV }\end{array}$ & $\begin{array}{l}\text { Sunitinib } \\
\text { Sorafenib } \\
\text { Placebo }\end{array}$ & 1 year & DFS & $\begin{array}{l}5.8 \text { vs } \\
6.1 \text { years } \\
\text { HR } 0.97 \\
P=0.71\end{array}$ \\
\hline $\begin{array}{l}\text { SORCE: Sorafenib } \\
\text { in Patients with } \\
\text { Resected Primary } \\
\text { RCC at } \\
\text { High/Intermediate } \\
\text { Risk of Relapse }\end{array}$ & 1,711 & $\begin{array}{l}\text { Patients with high- } \\
\text { and intermediate- } \\
\text { risk resected RCC } \\
\text { according to } \\
\text { Leibovich risk } \\
\text { assessment }\end{array}$ & $\begin{array}{l}\text { Sorafenib } \\
\text { Sorafenib/ } \\
\text { Placebo } \\
\text { Placebo }\end{array}$ & 3 years & DFS & $\begin{array}{l}\text { Median } \\
\text { Not } \\
\text { reached } \\
5 \text {-years } \\
\text { DFS 67\% } \\
\text { vs } 65 \% \\
\text { HR } 1.01 \\
p=0.95\end{array}$ \\
\hline $\begin{array}{l}\text { EVEREST: } \\
\text { Everolimus for } \\
\text { Renal Cancer }\end{array}$ & 1,545 & $\begin{array}{l}\text { Pathological stage } \\
\text { intermediate or very } \\
\text { high-risk patients }\end{array}$ & $\begin{array}{l}\text { Everolimus } \\
\text { Placebo }\end{array}$ & $\begin{array}{l}9 \\
\text { treatment } \\
\text { cycles }\end{array}$ & RFS & NA \\
\hline
\end{tabular}




\begin{tabular}{|c|c|c|c|c|c|c|}
\hline $\begin{array}{l}\text { Ensuing Surgical } \\
\text { Therapy }^{34}\end{array}$ & & $\begin{array}{l}\text { with full or partial } \\
\text { nephrectomy }\end{array}$ & & & & \\
\hline $\begin{array}{l}\text { PROTECT: } \\
\text { Pazopanib as an } \\
\text { Adjuvant } \\
\text { Treatment for } \\
\text { Localized } \text { RCC }^{35}\end{array}$ & 1,538 & $\begin{array}{l}\text { Patients with } \\
\text { moderately high or } \\
\text { high risk of relapse } \\
\text { with nephrectomy of } \\
\text { Localized or locally } \\
\text { advanced RCC }\end{array}$ & $\begin{array}{l}\text { Pazopanib } \\
\text { Placebo }\end{array}$ & $1 \mathrm{yr}$ & DFS & $\begin{array}{l}3 \text {-years } \\
\text { DFS } \\
67 \% \text { vs } \\
64 \% \\
\text { HR } 0.86 \\
P=0.16\end{array}$ \\
\hline $\begin{array}{l}\text { ATLAS: Adjuvant } \\
\text { Axitinib Therapy } \\
\text { of Renal Cell } \\
\text { Cancer in High } \\
\text { Risk Patients }^{36}\end{array}$ & 724 & $\begin{array}{l}\text { High-risk, non- } \\
\text { metastatic RCC with } \\
\text { nephrectomy }\end{array}$ & $\begin{array}{l}\text { Axitinib } \\
\text { Placebo }\end{array}$ & 3 yrs & DFS & $\begin{array}{l}\text { Stopped } \\
\text { due to } \\
\text { futility } \\
\text { HR } 0.87 \\
P=0.32\end{array}$ \\
\hline
\end{tabular}

Legend: UISS UCLA-Integrated Staging System, DFS disease-free survival, RFS recurrence-free survival, RCC renal cell carcinoma, HR hazard ratio

\section{LND templates}

LND as a prognostic tool would benefit from a standardized dissection template. However, the dynamics of lymphatic drainage in the retroperitoneum and its further lymphatic and lymphovenous connections are still poorly understood and a consensus regarding surgical LND templates does not exist. Historical studies of renal lymphatic drainage date back to 1935 when Parker conducted the first well established drainage study in kidneys [46]. In the description of radical nephrectomy prevailing in the 1960s and 70s, Robson suggested removing para-aortic and paracaval lymph nodes from the bifurcation of the aorta to the crus of the diaphragm $[47,48]$. In more contemporary studies, Crispen et al. suggested that in high risk tumours on the right side the paracaval and interaortocaval lymph nodes should be removed from the crus to the ipsilateral common iliac artery, whereas for tumours on the left side the paraaortic and interaortocaval lymph nodes should be dissected, using the same ipsilateral upper and lower boundaries as on the right side [49].

Futhermore, a systematic review described most commonly used LND templates which included on the right side the hilar, paracaval, and precaval nodes, and on the left side the hilar, pre-paraaortic nodes, both from the crus of the diaphragm to the aortic bifurcation [50]. However, in most of the studies LND templates were unstandardized and performed according to surgeons preference. One of the explanation for the absence of standardized LND templates is a lack of understanding of lymphatic drainage in RCC. Also, conflicting results in LND harms and benefits have added to the gaps in LND studies and practice. 
LND templates are determined by lymphatic drainage pattern and the location of first landing sites of lymph node metastases. However, most of the surgical studies from which data for LND templates were extracted had been based on resection of multiple lymph node metastases in the retroperitoneum which prevents analysis which of these were the first draining nodes. Knowledge of the location of the first nodes receiving drainage from the tumour is vital to develop LND templates. Furthermore, studies are lacking despcriptions of precise anatomical sites, numbers of lymph nodes resected and information on indication and extent of LND [50]. In addition, surgical mapping studies have limited value in assessing lymphatic drainage as it is only possible to assess what has been exposed and removed. To add to the uncertainty, cadaveric studies in humans with blue dye injected into Gerotas fascia revealed direct connections of renal lymphatics in the retroperitoneum to the thoracic duct without intervening lymph nodes in $23 \%$ on the left and $38 \%$ on the right side [51]. All these limitations make establishing LND templates for renal cell carcinoma challenging.

Consequently, there is a need for a proper lymphatic drainage study with modern methods allowing dynamic in vivo-imaging.

Detection of lymph node metastases with cross-sectional imaging has a sensitivity of $77 \%$, specificity of $73 \%$ and a positive predictive value of $29 \%$, all of which are low with the exception of a negative predictive value of $96 \%$ [48]. These limitations have encouraged us and others to explore lymphatic drainage of renal tumours with sentinel lymph node imaging technology [52]. The aim of sentinel node detection by dynamic imaging is to map the first landing sites of the radiotracer injected into the primary tumour which would in theory represent the first lymph nodes of the regional lymph node basin to receive metastatic cells before they sequentially spread through the lymphatics to other nodes or through lymphovenous connections to distant organ sites [53]. Sentinel node resection has also the advantage of detecting occult micrometastatic nodal disease which may result in more accurate staging avoiding the potential surgical adverse events of extended LND.

The aim of the thesis and the primary endpoint of the main trial is to prospectively map the sentinel nodes in renal tumours with dynamic lymphoscintigraphy and single-photon emission computed tomography SPECT/CT imaging to evaluate the first draining lymph nodes. Secondary and exploratory endpoints were to assess sentinel lymph node biopsy outcome, surgical technique and safety and finally to analyse non-visualization. To complete the topic we assess if occult or clinically limited single site lymph node metastases are located topographically at the sites observed in the prospective image-based sentinel node mapping study. In conclusion with the compiled data we describe the lymphatic drainage in renal cancer and also suggest a LND template which could be used for prognosis assessment and in future studies.

\section{Outline of the thesis}

The thesis contains eight chapters. As an introduction to current clinical-medical research for advanced lymph node metastatic renal cancer, Chapter 1 reviews the paradigm change in systemic therapy for renal cell carcinoma, including trials of immune checkpoint inhibitor treatment as adjuvant therapy for patients with high-risk disease for which assessment of lymph node metastasis is of prognostic significance. Chapter $\mathbf{2}$ is reporting the primary endpoint of a prospective phase II study to evaluate the topographic distribution of renal tumour draining sentinel lymph nodes on scintigraphy and SPECT/CT imaging. Chapter $\mathbf{3}$ analyzes the outcome of sentinel lymph node biopsy 
which was the secondary endpoint of the prospective phase II imaging study. Chapter $\mathbf{4}$ reports on durable survival with papillary type II renal cell carcinoma and lymph node metastases of a patient who was enrolled in the prospective sentinel node study. Chapter 5 assesses retrospectively the topographic distribution of occult or clinically limited single-site lymph node metastases in renal cancer and evaluates if these match with the locations observed in the sentinel node imaging studies. Chapter 6 is analyzing the causes for non-visualization of sentinel lymph nodes on scintigraphy and SPECT/CT imaging. Chapter $\mathbf{7}$ is a report on surgical safety and morbidity of sentinel lymph node biopsy. Also technical details are described and discussed. Chapter 8 summarizes all the studies and gives a perspective and future outlook.

\section{References}

1. Znaor A, Lortet-Tieulent J, Laversanne M, Jemal A, Bray F. International variations and trends in renal cell carcinoma incidence and mortality. Eur Urol. 2015;67(3):519-30.

2. Siegel RL, Miller KD, Jemal A. Cancer statistics, 2016. CA Cancer J Clin. 2016;66:7-30.

3. Sun M, Shariat SF, Cheng C, Ficarra V, Murai M, Oudard S, et al. Prognostic factors and predictive models in renal cell carcinoma: a contemporary review. Eur Urol. 2011;60(4):644-61.

4. Cancer incidence and mortality worldwide 2018: IARC Cancerbase No. 11. Lyon, France: International Agency for Research on Cancer;2018.

5. Ebele JN, Sauter G, Epstein JI, Sesterhenn IA. Pathology and genetics of tumours of the urinary system and male genital organs. World Health Organization classification of tumours. Lyon, France:International Agency of Research on Cancer;2004.

6. Amin MB, Edge S, Greene F, Byrd DR, Brookland RK, Washington MK, et al. AJCC Cancer Staging Manual. 8th ed. Switzerland: Springer, 2017

7. Chandrasekar T, Klaassen Z, Goldberg H, Kulkarni GS, Hamilton RJ, Fleshner NE. Metastatic renal cell carcinoma: Patterns and predictors of metastases-A contemporary population-based series.Urol Oncol. 2017;35(11):661.e7-661.e14.

8. Ljungberg B, Albiges L, Abu-Ghanem Y, Bensalah K, Dabestani S, Fernández-Pello S, et al. European Association of Urology Guidelines on Renal Cell Carcinoma: The 2019 Update. Eur Urol. 2019;75(5):799-810.

9. Wong MCS, Goggins WB, Yip BHK, Fung FDH, Leung C, Fang Y, et al. Incidence and mortality of kidney cancer: temporal patterns and global trends in 39 countries. Sci Rep. 2017;7(1):15698.

10.Méjean A, Ravaud A, Thezenas S, Colas S, Beauval JB, Bensalah K, et al. Sunitinib Alone or after Nephrectomy in Metastatic Renal-Cell Carcinoma. N Engl J Med. 2018;379(5):417-27.

11. Bex A, Mulders P, Jewett M, Wagstaff J, van Thienen JV, Blank CU et al. Comparison of Immediate vs Deferred Cytoreductive Nephrectomy in Patients with Synchronous Metastatic Renal Cell Carcinoma Receiving Sunitinib: The SURTIME Randomized Clinical Trial. JAMA Oncol. 2019;5(2):16470 . 
12. Kim JH, Li S, Khandwala Y, Chung KJ, Park HK, Chung BI. Association of Prevalence of Benign Pathologic Findings After Partial Nephrectomy With Preoperative Imaging Patterns in the United States From 2007 to 2014. JAMA Surg. 2019; 154(3):225-31.

13. Pantuck AJ, Zisman A, Dorey F, Chao DH, Han KR, Said J et al. Renal cell carcinoma with retroperitoneal lymph nodes: role of lymph node dissection. J Urol. 2003;169:2076-83.

14. Terrone C, Cracco C, Porpiglia F, Bollito E, Scoffone C, Poggio M, et al. Reassessing the current TNM lymph node staging for renal cell carcinoma. Eur Urol. 2006;49(2):324-31.

15. Blute ML, Leibovich $\mathrm{BC}$, Cheville JC, Lohse $\mathrm{CM}$, Zincke $\mathrm{H}$. A protocol for performing extended lymph node dissection using primary tumor pathological features for patients treated with radical nephrectomy for clear cell renal cell carcinoma. J Urol. 2004;172(2):465-9.

16. Gershman B, Moreira DM, Thompson RH, Boorjian SA, Lohse CM, Costello BA, et al. Renal Cell Carcinoma with Isolated Lymph Node Involvement: Long-term Natural History and Predictors of Oncologic Outcomes Following Surgical Resection. Eur Urol. 2017;72(2):300-6.

17. Blom JHM, Van Poppel H, Maréchal JM, Jacqmin D, Schröder FH, de Prijck L, et al. Radical Nephrectomy with and without Lymph-Node Dissection: Final Results of European Organization for Research and Treatment of Cancer (EORTC) Randomized Phase 3 Trial 3088. Eur Urol. 2009;55:28-34.

18. Bhindi B, Wallis CJD, Boorjian SA, Thompson RH, Farrell A, Kim SP, et al. The role of lymph node dissection in the management of renal cell carcinoma: a systematic review and meta-analysis. BJU Int. 2018;121(5):684-98.

19. Whitson JM, Harris CR, Reese AC, Meng MV. Lymphadenectomy improves survival of patients with renal cell carcinoma and nodal metastases. J Urol. 2011;185:1615-20.

20. Capitanio U, Suardi N, Matloob R, Roscigno M, Abdollah F, Di Trapani E, et al. Extent of lymph node dissection at nephrectomy affects cancer-specific survival and metastatic progression in specific sub-categories of patients with renal cell carcinoma (RCC). BJU Int 2014; 114: 210-15.

21. Gershman B, Thompson RH, Boorjian SA, Larcher A, Capitanio U, Montorsi F, et al. Radical Nephrectomy with or without Lymph Node Dissection for High Risk Nonmetastatic Renal Cell Carcinoma: A Multi-Institutional Analysis. J Urol. 2018;199(5):1143-48.

22. Turajlic S, Xu H, Litchfield K, Rowan A, Chambers T, Lopez Jl, et al. Tracking Cancer Evolution Reveals Constrained Routes to Metastases: TRACERx Renal. Cell. 2018;173(3):581-594.e12.

23. Capogrosso P, Larcher A, Nini A, Muttin F, Cianflone F, Ripa F, et al. The critical role of lymph node dissection in selecting high-risk nonmetastatic renal cancer candidates for adjuvant therapy after nephrectomy. Urol Oncol. 2019;37(4):293.e25-293.e30.

24. Golijanin B, Pereira J, Mueller-Leonhard C, Golijanin D, Amin A, Mega A, Boorjian SA, et al. The natural history of renal cell carcinoma with isolated lymph node metastases following surgical resection from 2006 to 2013. Urol Oncol. 2019;37(12):932-40.

25. Leibovich BC, Blute ML, Cheville JC, Lohse CM, Frank I, Kwon ED et al. Prediction of progression after radical nephrectomy for patients with clear cell renal cell carcinoma: a stratification tool for prospective clinical trials. Cancer. 2003;97:1663-71. 
26. Leibovich BC, Lohse CM, Cheville JC, Zaid HB, Boorjian SA, Frank I, et al. Predicting Oncologic Outcomes in Renal Cell Carcinoma After Surgery Eur Urol. 2018;73(5):772-80.

27. Sorbellini M, Kattan MW, Snyder ME, Reuter V, Motzer R, Goetzl M, et al. A postoperative prognostic nomogram predicting recurrence for patients with conventional clear cell renal cell carcinoma. J Urol. 2005;173:48-51.

28. Kattan MW, Reuter V, Motzer RJ, Katz J, Russo P. A postoperative prognostic nomogram for renal cell carcinoma. J Urol. 2001;166(1):63-7.

29. Frank I, Blute ML, Cheville JC, Lohse CM, Weaver AL, Zincke H. An outcome prediction model for patients with clear cell renal cell carcinoma treated with radical nephrectomy based on tumor stage, size, grade and necrosis: the SSIGN score. J Urol. 2002;168(6):2395-400.

30. Zisman A, Pantuck AJ, Wieder J, Chao DH, Dorey F, Said JW, deKernion JB, Figlin RA, Belldegrun AS. Risk group assessment and clinical outcome algorithm to predict the natural history of patients with surgically resected renal cell carcinoma. J Clin Oncol. 2002; 20(23):4559-66.

31. Ravaud A, Motzer RJ, Pandha HS, George DJ, Pantuck AJ, Patel A, et al. Adjuvant Sunitinib in HighRisk Renal-Cell Carcinoma after Nephrectomy. N Engl J Med. 2016;375(23):2246-54.

32. Haas NB, Manola J, Uzzo RG, Flaherty KT, Wood CG, Kane C et al. Adjuvant sunitinib or sorafenib for high-risk, non-metastatic renal-cell carcinoma (ECOG-ACRIN E2805): a double-blind, placebocontrolled, randomised, phase 3 trial. Lancet. 2016;387:2008-16

33. Eisen T, Frangou E, Smith B, Ritchie A, Kaplan R, Oza B, et al. Primary Efficacy analysis results from the SORCE trial (RE05): Adjuvant sorafenib for renal cell carcinoma at intermediate or high risk of relapse: an international, randomised double-blind phase III trial led by the MRC CTU at UCL. Annals of Oncology.2019;30(5):v851-v934.

34. clinicaltrials.gov NCT01120249

35. Motzer RJ, Haas NB, Donskov F, Gross-Goupil M, Varlamov S, Kopyltsov E. Randomized Phase III Trial of Adjuvant Pazopanib Versus Placebo After Nephrectomy in Patients With Localized or Locally Advanced Renal Cell Carcinoma. J Clin Oncol. 2017:10;35(35):3916-23.

36. Gross-Goupil M, Kwon TG, Eto M, Ye D, Miyake H, Seo SI, et al. Axitinib versus placebo as an adjuvant treatment of renal cell carcinoma: results from the phase III, randomized ATLAS trial. Ann Oncol. 2018;29(12):2371-8.

37. Haas NB, Manola J, Dutcher JP, Flaherty KT, Uzzo RG, Atkins MB, et al. Adjuvant Treatment for High-Risk Clear Cell Renal Cancer: Updated Results of a High-Risk Subset of the ASSURE Randomized Trial. JAMA Oncol. 2017;3(9):1249-52.

38. Motzer R.J, Tannir N.M, McDermott D.F, Arén Frontera O, Melichar B, Choueiri TK, et al. Nivolumab plus Ipilimumab versus Sunitinib in Advanced Renal-Cell Carcinoma. N Engl J Med. 2018;378(14):1277-90.

39. Rini BI, Plimack ER, Stus V, Gafanov R, Hawkins R, Nosov D et al. Pembrolizumab plus Axitinib versus Sunitinib for Advanced Renal-Cell Carcinoma. N Engl J Med. 2019;380(12):1116-27. 
40. Zhang T, Zhu J, George D J, Nixon AB. Metastatic clear cell renal cell carcinoma: Circulating biomarkers to guide antiangiogenic and immune therapies. Urologic Oncology: Seminars and Original Investigations. 2016;34(11):510-18.

41. clinicaltrials.gov NCT03055013

42. clinicaltrials.gov NCT03024996

43. clinicaltrials.gov NCT03142334

44. clinicaltrials.gov NCT03288532

45. clinicaltrials.gov NCT03138512

46. Parker AE. Studies on the main posterior lymph channels of the abdomen. Am J Anat.1935;56:409-43.

47. Robson CJ, Churchill BM, Anderson W. The results of radical nephrectomy for renal cell carcinoma. J Urol.1969;101:297-301.

48. Zareba P, Pinthus JH, Russo P. The contemporary role of lymph node dissection in the management of renal cell carcinoma. Ther Adv Urol. 2018; 10(11): 335-42.

49. Crispen PL, Breau RH, Allmer C, Lohse CM, Cheville JC, Leibovich BC et al. Lymph node dissection at the time of radical nephrectomy for high-risk clear cell renal cell carcinoma: indications and recommendations for surgical templates. Eur Urol.2011;59:18-23.

50. Campi R, Sessa F, Di Maida F, Greco I, Mari A, Takáčová T, et al. Templates of Lymph NodeDissection for Renal Cell Carcinoma: A Systematic Review of the Literature. Front Surg. 2018;5:76.

51. Assouad J, Riquet M, Foucault C, Hidden G, Delmas V. Renal lymphatic drainage and thoracic duct connections: implications for cancer spread. Lymphology. 2006;39(1):26-32.

52. Sherif AM, Eriksson E, Thörn M, Vasko J, Riklund K, Ohberg L, Ljungberg BJ. Sentinel node detection in renal cell carcinoma. A feasibility study for detection of tumour-draining lymph nodes. BJU Int. 2012;109(8):1134-1139.

53. Karmali RJ, Suami H, Wood CG, Karam JA. Lymphatic drainage in renal cell carcinoma: back to the basics. BJU Int. 2014;114(6):806-17. 


\section{Chapter 1}

Antiangiogenic therapy combined with immune checkpoint blockade in renal cancer

Teele Kuusk, Laurence Albiges, Bernard Escudier, Nikolaos Grivas, John Haanen,Thomas Powles, Axel Bex

Angiogenesis. 2017 May;20(2):205-215. 


\section{Abstract}

Antiangiogenic therapy with vascular endothelial growth factor (VEGF) inhibitors is the current first line treatment in metastatic renal cell carcinoma (mRCC). Immunotherapy with checkpoint inhibitor, has been recently added to the armamentarium of mRCC treatment. These therapies are based on treatment with antibodies that block programmed cell death-1 (PD-1), programmed cell death ligand 1 (PD-L1) pathways, demonstrating impressive response rates and improved survival in several tumour types. So far, nivolumab is the only approved anti-PD1 monoclonal antibody after VEGF therapy in mRCC. According to preclinical and clinical studies, combination therapies with VEGF- and checkpoint-inhibitors have synergistic effect achieving improved response rates. However, toxicity in some combinations is high. In this article we present a review of the ongoing trials with these drug combinations for RCC.

\section{Introduction}

Renal cell carcinoma (RCC) represents $5 \%$ and $3 \%$ of all malignancies in men and women, respectively [1,2]. In Europe, the incidence and mortality is approximately 85/100.000 and $35 / 100.000$, respectively [3]. Fifteen \% of the patients with primary RCC are diagnosed with metastatic disease, while $30 \%$ of initially locally treated patients develop recurrent disease and systemic progression during the course of the disease [3]. Systemic therapy with vascular endothelial growth factor (VEGF) signaling axis targeting agents is the first line treatment for metastatic RCC (mRCC) [4,5]. In addition to established first- and second-line molecular targeted therapies, immunotherapeutic agents are introduced into the treatment algorithm and are currently actively studied. In 2015, nivolumab was the first immune checkpoint inhibitor to be approved by the Food and Drug Administration (FDA) and European Medicine Agency (EMEA), as second line treatment for mRCC.

Neoangiogenesis and immune system play a central role in RCC. The earliest proof for the essential role of VEGF in RCC pathogenesis came from understanding of the genetic basis of the von Hippel-Lindau (VHL) familial syndrome [6]. Later studies showed the impact of VHL gene mutations on the upregulation of VEGF and expression of other angiogenic factors, which are of significance in RCC development and progression [7]. Early observations of spontaneous regression of metastases after radical nephrectomy, suggested an importance of the immune system in RCC. The main cause of this regression was believed to be a T- and B-cell mediated 
antitumour immunity [8]. However, with the exception of high-dose intravenous interleukin-2 (IL-2), treatment with cytokines such as interferon- $\alpha$ or subcutaneous IL-2 had only modest activity [9]. Despite a consistent rate of 5-10\% of patients being in complete remission and potentially cured after high-dose IL-2, the high adverse event rate and the inability to predict responders did not favour this treatment option. After the introduction of VEGF-targeted therapy for the treatment of clear-cell RCC, combinations of these drugs with cytokines have been studied [10]. Unfortunately, with the exception of bevacizumab and interferon- $\alpha$, combinations were either ineffective or too toxic. The lower adverse event rate seen with PD1/PDL1 inhibitors has led to a revival in the investigation of combinations of drugs acting on VEGF and immune checkpoint inhibition in MRCC. This rationale is further supported by the observation that antiangiogenic agents have an effect on antitumour immune responses and T cell trafficking to the tumour $[11,12]$. It has also been shown that checkpoint inhibition modulates tumour vessels [13]. Combining agents that act on these two major oncogenic pathways synergistically may result in better response and potential benefit from these therapies. In this article we review the current literature and ongoing trials on combination therapies of VEGF-tyrosine kinase inhibitors (TKI), VEGF-monoclonal antibodies (mAB) and immunotherapeutic agents (checkpoint inhibitors) for RCC.

\section{Mechanism of action of VEGF and checkpoint inhibitors in RCC}

Inactivation of VHL tumour suppressor gene induces hypoxia which in turn triggers hypoxiainducible factor (HIF)-1, causing activation of pro-angiogenic factors. VEGF upregulation results in neoangiogenesis, which facilitates the access of tumor cells to the general circulation causing systemic disease [14]. Tumour angiogenesis enhances activity of myeloid derived suppressor cells (MDSC) and tumour-associated macrophages (TAM) suppressing innate antitumour immunity. It has been demonstrated that VEGF-receptor tyrosine kinase inhibitor (VEGFR-TKI) sunitinib is suppressing angiogenic genes resulting in inhibition of angiogenesis in pretreated primary tumour tissue [15]. In preclinical models, it has been shown that antiangiogenic therapy decreased MDSC and reprogrammed immunomodulatory phenotype of TAM. The evolution of VEGFR-TKIs namely sunitinib, pazopanib, sorafenib, axitinib, cabozantinib, lenvatinib in combination with mTOR inhibitor (everolimus) and monoclonal antibody against VEGF (bevacizumab) in combination with interferon- $\alpha$, have improved mRCC prognosis by increasing progression free survival (PFS) and impacting on overall survival (OS) [16-21]. Currently, sunitinib, pazopanib and bevacizumab with interferon- $\alpha$ are first line 
options while nivolumab, cabozantinib, axitinib, sorafenib, everolimus alone and combination with lenvatinib are second line treatment options in clear-cell mRCC $[4,5]$.

Reciprocal action between the immune system and tumour development and progression have been a challenging topic in immunology. It is well known that the immune system prevents cancer development in many different pathways. However, cancer cells have also mechanisms against host immune system activity. At first, the innate and adaptive immune system both cooperate to eradicate tumor cells before clinically detectable disease [22]. After that, the adaptive system continues its attack against tumor cells, which survive. However, tumor cell types finally develop that are not recognized by the adaptive immune system. This happens through different mechanisms: tumour cells can become insensitive to immune effector mechanisms or immune checkpoint proteins may become dysregulated, typically via expression of inhibitory ligands and receptors that regulate $T$ cell effector functions in the tumor microenvironment. This induces an immunosuppressive tumor microenvironment resulting in the escape phase, where tumour development is not prevented by the host immune system leading ultimately to clinically detectable disease [22].

Normally, microbes as well as cancer cells evoke activation of the immune system and in this process immune checkpoints are protecting the host cells from autoimmunity and selfdestruction. Cancer cells are able to co-opt immune checkpoint pathways and thus avoid immune eradication. Therefore, immune checkpoint inhibitory antibodies act on tumour cells indirectly by targeting lymphocyte receptors or their ligands for re-activating and enhancing internal antitumour immunity. Checkpoint receptors are expressed on T-lymphocytes (CTLA4) and on T-, B-lymphocytes and natural killer (NK) cells such as programmed death-1 receptor (PD-1) and programmed death ligand 1 (PD-L1). Immune checkpoint blockade with monoclonal antibodies target and block these inhibitory receptors, thereby inducing immune responses at different levels [ 23,24,25]. Pembrolizumab and nivolumab target the PD-1 receptor while atezolizumab, avelumab and durvalumab block its ligand (PD-L1). Ipilimumab and tremelimumab target CTLA-4 $[25,26]$. Nivolumab has shown an OS benefit compared to everolimus in patients with $\mathrm{mRCC}$ previously treated with antiangiogenic therapy and is currently the only approved checkpoint inhibitor for the treatment of mRCC [27].

\section{Rationale for using combination of antiangiogenic agents and immunotherapy}

Earlier studies have shown that anti angiogenic therapy can elicit or enhance antitumour immunity whereas reciprocally the immune system can induce angiogenesis $[23,28,29]$. Therefore, there 
is a bidirectional link and synergy between antiangiogenic agents and immunotherapy [28] (Figure 1). Antiangiogenic agents are capable to reverse immunosuppression by decreasing immunosuppressive cells (MDSCs, regulatory T cells), immunosuppressive cytokines (IL-10, TGF $\beta$ ) and inhibitory molecules on T cells (PD-1) [28]. Moreover, VEGF receptor inhibitors drive tumour cells to activate immune checkpoints and therefore a combination of VEGF- and checkpoint inhibitors makes sense $[23,29]$. Combination of anti-VEGF therapy with immunotherapy, though not checkpoint inhibitors, has demonstrated improved PFS in mRCC already in 2007 in two trials of bevacizumab in combination with interferon- $\alpha$ leading to approval as a first-line therapeutic option in $\mathrm{mRCC}[10]$. In addition, recent research on intratumoral immune components such as tumour infiltrating lymphocytes (TIL) or MDSCs in tumour tissue of sunitinib pretreated primary RCC have demonstrated potential synergism for TKI with anti-PD-(L)1 therapy [30]. Pretreatment with sunitinib improved TIL expansion by reduction in intratumoral content of MDSC. Furthermore, the function of tumour infiltrating $T$ lymphocytes may be inhibited in an immunosuppressive tumour microenvironment by $T$ regulatory cells and expression of PD-L1. It has been shown that patients treated with antiangiogenic therapy have increased Treg and PD-L1 expression in their primary tumour tissue and this is associated with poor survival. Thus, combination therapy may be effective for patients with mRCC [31]. Recently published translational and clinical data on the combination of bevacizumab with atezolizumab (anti-PD-L1) in 10 patients demonstrated that combination therapy improves antigen-specific T-cell migration thus enhancing antitumour activity. Durable partial responses (PR) and stable disease (SD) were observed in 8 patients. This durable clinical benefit may be due to an addition of dissimilar response kinetics, since VEGFR-TKIs produce fast but non-durable response, but PD-1 inhibitors are slow to act but the response is long-lasting and thorough [32].

\section{Combination therapy trials in advanced and metastatic RCC}

Several trials have been performed or are ongoing to assess different combinations of antiangiogenic agents with checkpoint inhibitors in RCC. A phase I study (Checkmate-016, NCT01472081) in mRCC compared combination therapy of nivolumab, an anti-PD-1 inhibitor, with sunitinib, pazopanib or ipilimumab [33] (Tabel 2,3). Starting dose for nivolumab was 2 $\mathrm{mg} / \mathrm{kg}$ (maximum $5 \mathrm{mg} / \mathrm{kg}$ ) intravenously every 3 weeks until progressive disease (PD), toxicity or other reason for discontinuation, while standard dose for sunitinib and pazopanib was 50 $\mathrm{mg}$ and $800 \mathrm{mg}$, respectively. Primary outcome measures of the study were safety and 
tolerability of the different combinations while secondary outcome were the objective response rate (ORR) and the duration of response. In the sunitinib and nivolumab arm no doselimiting toxicities (DLT) were seen and the arm with higher dose $(5 \mathrm{mg} / \mathrm{kg}$ ) of nivolumab was expanded (up to 33 patients). The nivolumab ( $2 \mathrm{mg} / \mathrm{kg}$ )-pazopanib combination arm (20 patients) was closed due to early DLT. Moreover, adverse event rate was high with both combinations. A $82 \%$ and $70 \%$ rate of grade 3-4 toxicity was seen in the nivolumab-sunitinib and nivolumab-pazopanib arm, respectively. The most common grade 3-4 adverse events for the nivolumab-sunitinib and-pazopanib combination were liver enzymes rise, hypertension, hyponatremia and lymphocytopenia. Regarding the effectiveness, nivolumab-sunitinib and nivolumab-pazopanib combinations showed ORR of $52 \%$ and $45 \%$, respectively. The response was seen 6 weeks after treatment initiation in $41 \%$ and $56 \%$ in combinations of nivolumab with sunitinib and pazopanib, respectively, and demonstrated long lasting effects up to 13 and 17 months in the sunitinib- and pazopanib-nivolumab combinations, respectively. Median progression free survival (PFS) was 48.9 and 31.4 months for sunitinib and pazopanib combinations, respectively. This study showed higher response rates for combination therapy compared to monotherapy, although toxicity was higher.

A recently launched phase $\mathrm{I} / \mathrm{II}$ will be investigating the combination of nivolumab with tivozanib, a VEGFR-TKI in advanced RCC (TiNivo trial).

At least 5 trials investigate pembrolizumab, an anti-PD-1 inhibitor, in combinations with monoclonal antibodies against VEGF- or antiangiogenic VEGFR-TKI. Pembrolizumab (MK-3475) has been studied with the combination of bevacizumab in a phase Ib study [34]. Sixteen patients with mRCC who had at least one systemic therapy failure were enrolled. Pembrolizumab (200 mg every 3 weeks) was given in combination with bevacizumab (either at $10 \mathrm{mg} / \mathrm{kg}$ or $15 \mathrm{mg} / \mathrm{kg}$ every 3 weeks). No grade 3-4 AEs were recorded. Seventy-one \% of 14 patients who were evaluable for response demonstrated PR, $29 \%$ had PD. To conclude, pembrolizumab and bevacizumab at maximum dose was safe and recommended to continue in a phase II study (NCT02348008) BTCRC-GU14-003.

The other phase I study of a monoclonal antibody against VEGF, aflibercept, in combination with pembrolizumab enrolls patients with solid tumours and mRCC who have been previously treated with VEGFR-TKIs [35] (NCT02298959). They receive pembrolizumab and ziv-aflibercept intravenously on day 1 and cycles are repeated every 2 weeks. Results are pending.

Further combinations of pembrolizumab with VEGFR-TKI were investigated in phase Ib/II studies. One such trial enrolled 8 RCC patients among other patients with solid tumours who had 
progressed after first-line therapy [36] to receive pembrolizumab (200 mg) intravenously once every 3 weeks and a daily oral dose of lenvatinib (24 mg or $20 \mathrm{mg}$ )(NCT02501096). Grade 3 adverse events with $24 \mathrm{mg}$ of lenvatinib were arthralgia and fatigue, however no DLTs were reported in the arm combining pembrolizumab and lenvatinib $20 \mathrm{mg}$. ORR for this combination was $69 \%$. Half of the mRCC patients showed PR and the other half SD. The maximum daily tolerated dose of lenvatinib in the combination was confirmed as $20 \mathrm{mg}$ and a phase III study testing the combination against sunitinib, a first-line standard, is ongoing [37] (Table 1).

Interestingly, other VEGFR-TKI combinations with pembrolizumab may not be necessarily comparable regarding their toxicity profile. Another phase I/II study combined pembrolizumab with pazopanib,600 or 800 mg [38] (Keynote-018, NCT02014636). Sixty-five \% of patients developed grade 3 hepatotoxic AEs and toxicity appeared recurrently after re-initiation of treatment. The investigators concluded that liver function deterioration was related to pazopanib. ORR were $60 \%$ and $20 \%$ for pazopanib $800 \mathrm{mg}$ and $600 \mathrm{mg}$, respectively. One patient in the pembrolizumab-pazopanib $800 \mathrm{mg}$ arm showed complete response (CR).

Finally, a phase Ib study investigated pembrolizumab in combination with axitinib in 52 treatmentnaive patients. The trial determined that the safe dose of axitinib was $5 \mathrm{mg}$ twice daily and 2 $\mathrm{mg} / \mathrm{kg}$ every 3 weeks for pembrolizumab [39]. Severe grade 3-4 adverse events included hypertension, diarrhea and headache. Seventy-one $\%$ of the patients obtained objective response, with 3 CR 34 PR and 10 had SD.

Axitinib was further investigated in a phase Ib study which evaluated the safety, pharmacokinetics and pharmacodynamics of axitinib ( 3 or $5 \mathrm{mg}$ twice a day) in combination with the anti PD-L1 inhibitor avelumab (10 mg/kg every 2 weeks) in first-line advanced RCC [40]. Grade 3-4 adverse events occurred in 5/6 patients, hypertension being the most common one. No discontinuation due to treatment related toxicity was observed. Confirmed PR was observed in 6 patients. The dose combination with avelumab and axitinib regarded as safe was $10 \mathrm{mg} / \mathrm{kg}$ and $5 \mathrm{mg}$, respectively. Both pembrolizumab and avelumab combinations with axitinib were considered encouraging and are currently being tested in phase 3 trials against the standard sunitinib in untreated mRCC $[41,42]$ (Table 1$)$.

Like the anti-PD-1 inhibitor pembrolizumab, atezolizumab, another monoclonal antibody against PD-L1, has been studied in combination with bevacizumab in phase I and II studies $[43,44]$. In a phase I study atezolizumab ( $20 \mathrm{mg} / \mathrm{kg}$ every 3 weeks) was administered with bevacizumab $(15 \mathrm{mg} / \mathrm{kg}$ every 3 weeks) in 12 patients. Atezolizumab related grade 3 adverse events occurred 
in $3 \%$ of the patients, however grade $3-4$ AEs accounted for $58 \%$. ORR was observed in $40 \%, 1$ patient had a CR and almost half of the patients experienced SD. These results suggested a safety and efficacy of the combination in $\mathrm{MRCC}$ which led to a randomized phase II study. In the phase II study atezolizumab was administered either as monotherapy (103 patients) or in combination with bevacizumab (101 patients) versus sunitinib (101 patients) in patients with previously untreated locally advanced or metastatic RCC (IMmotion150, NCT01984242). This trial provides the first randomized data of VEGFR-TKI versus single agent PD-L1 inhibitor in first line. The results were encouraging especially in patients with higher expression of PD-L1 on immune cells and were presented at the 2017 American Society of Clinical Oncology Genitourinary (ASCO GU) symposium recently. ORR ranged from $32 \%, 25 \%, 29 \%$ in the atezolizumabbevacizumab combination, the atezolizumab-mono and sunitinib arm, respectively. Interestingly, complete response rate was the highest in the atezolizumab arm (11\%) followed by the combination (7\%) and sunitinib arm (5\%), respectively. Diverse response rates were seen in patients with higher expression of PD-L1 favouring the combination or atezolizumab only arms. As with previous combinations, AE rate was high: $64 \%, 41 \%$ and $69 \%$ of patients developed grade 3-4 AEs in the combination-, atezolizumab only and sunitinib arm, respectively. Atezolizumab only arm side effects were similar to side effects reported for nivolumab. There were one treatment related AE leading to death in the combination arm and 2 in the sunitinib arm. Promising results from the phase $1 /$ II studies have led this combination to a phase III study in which patients with treatment naïve mRCC were randomized to atezolizumab with or without bevacizumab versus sunitinib monotherapy (IMmotion 151, NCT02420821) [45] (Table 1). Atezolizumab and bevacizumab combination will also be further studied in phase 1 trials with entinostat, histone benzamide deacetylase inhibitor (NCT03024437) and obinutuzumab, anti CD20 monoclonal antibody (NCT03063762). Both trials have been registered early this year.

A phase I study examining the combination of tremelimumab, a CTLA-4 inhibitor, with sunitinib enrolled $28 \mathrm{mRCC}$ who had received none or only one previous systemic treatment [46]. The patients were treated with tremelimumab (6-15 mg/kg intravenously) once every 12 weeks and sunitinib ( $50 \mathrm{mg}$ daily on 4 on 2 off weeks schedule or $37.5 \mathrm{mg}$ continuously). Two of 5 patients in $50 \mathrm{mg}$ sunitinib plus tremelimumab $(6 \mathrm{mg} / \mathrm{kg})$ arm experienced DLTs, resulting in closure of the sunitinib $50 \mathrm{mg}$ dose arm. Half of the patients on sunitinib 37.5 and tremelimumab (15 mg/kg) developed DLTs. One patient receiving tremelimumab $(10 \mathrm{mg} / \mathrm{kg}$ ) plus daily sunitinib $(37.5 \mathrm{mg})$ died. Finally, the tremelimumab $(10 \mathrm{mg} / \mathrm{kg})$ plus daily sunitinib (37.5 mg) combination was expanded with 7 patients and 3 of those experienced DLTs. The 
most common DLT was acute renal failure. Finally, ORR was $76 \%$. However, due to high toxicity of renal failure, tremelimumab doses higher than $6 \mathrm{mg} / \mathrm{kg}$ combined with sunitinib (37.5 mg) were not recommended and not further studied

In comparison to the multitude of studies performed or ongoing for clear-cell RCC only two studies are currently enrolling patients with non-clear cell subtypes. Specifically, a phase II trial with atezolizumab and bevacizumab combination is accruing patients with advanced or metastatic non-clear cell RCC. Both drugs will be administered intravenously every 3 weeks. Results are awaited [47] (NCT02724878). Another combination trial is a phase Ib trial of durvalumab, a PD-L1 inhibitor, in combination with either savolitinib, a selective c-MET-TKI, or tremelimumab which enrolls a papillary RCC cohort in VEGFR-TKI refractory mRCC patients (CALYPSO, NCT02819596)[48].

The only triple combination trial is a phase I study in pretreated metastatic genitourinary cancer patients which compared combination therapy of nivolumab with cabozantinib and a triple combination of cabozantinib, nivolumab and ipilimumab. Forty patients with genitourinary cancers, among whom three patients with $\mathrm{mRCC}$, were enrolled. Preliminary results were presented at the 2017 American Society of Clinical Oncology Genito-urinary (ASCO GU) symposium (NCT02496208). Grade 3-4 AEs were hypophosphatemia, hyponatremia, elevated lipase. The combination of nivolumab and cabozantinib was well tolerated and did not cause grade 4-5 toxicities, immune-related AEs or DLTs. There were no additive toxicities also in a triple arm. Nivolumab $3 \mathrm{mg} / \mathrm{kg}$ every 2 weeks with cabozantinib $40 \mathrm{mg}$ daily and nivolumab 3 $\mathrm{mg} / \mathrm{kg}$, cabozantinib $40 \mathrm{mg}$ and ipilimumab $1 \mathrm{mg} / \mathrm{kg}$ was recommended to proceed to a phase II study. ORR in 38 evaluable patients for this combination was $32 \%$, and one of the $3 \mathrm{mRCC}$ patient had a PR [49].

In conclusion, given the many potential immune checkpoint inhibitor combinations with VEGFtargeted therapy that have been or are currently investigated in early phase $1 /$ II trials it may not come as a surprise that no less than 4 such combination trials are currently being investigated in treatment naïve $\mathrm{MRCC}$ patients challenging sunitinib, a first-line standard of care, in randomized controlled phase III settings [50] (Table 1). From these studies the IMmotion 151 trial has finished accrual and data may be presented as early as autumn 2017. 


\section{Combinations in neoadjuvant or presurgical setting in $\mathrm{mRCC}$}

Neoadjuvant or presurgical studies are a unique opportunity to obtain sequential tumour tissue and to identify predictors of response or resistance to immune checkpoint inhibition and combination with VEGF-targeted therapy. Preclinical and early clinical research suggests that there is significantly greater therapeutic efficacy of neoadjuvant immunotherapies in eradicating early occult metastases than with an adjuvant approach, following primary tumor resection [51]. This has resulted in several neoadjuvant and presurgical phase $1 /$ II studies in localized and metastatic RCC with single-agent nivolumab and pembrolizumab which are currently ongoing [52,53,54]. In addition, a phase III trial schedules patients for perioperative nivolumab before nephrectomy for $\geq T 2$ or $T$ any $N+R C C$ and plans to enroll 766 patients (PROSPER EA8143). There is one pilot randomized study evaluating presurgical nivolumab monotherapy, nivolumab combination with bevacizumab and nivolumab combined with ipilimumab in patients with primary $\mathrm{mRCC}$ and the tumour in place [55]. This is currently the only study investigating a combination of checkpoint inhibition and antiangiogenic therapy prior to removal of the primary tumour. One arm receives nivolumab $(3 \mathrm{mg} / \mathrm{kg}$ intravenously every 2 weeks for a total of 6 weeks). The second arm receives nivolumab ( $3 \mathrm{mg} / \mathrm{kg}$ every 2 weeks) with bevacizumab (10 mg/kg intravenosuly every 2 weeks for 6 weeks) and the third arm receives nivolumab ( $3 \mathrm{mg} / \mathrm{kg}$ every 3 weeks) with ipilimumab (1 $\mathrm{mg} / \mathrm{kg}$ intravenously every 3 weeks for 6 weeks). In all arms cytoreductive nephrectomy is planned after the end of drug treatment.

\section{Combination treatment in adjuvant setting}

Based on the assumption that immune checkpoint inhibition may be more effective in eliminating circulating tumour cells and micrometastases than VEGFR-targeted therapy, several randomized controlled phase 3 trials are planned to test adjuvant atezolizumab, nivolumab and pembrolizumab as single-agents in patients with non-metastatic RCC and high-risk of recurrence $[56,57,58,59]$. However, at present no combinations of immune checkpoint inhibition and VEGF-targeted therapy are being tested in the adjuvant setting. This is in part owing to conflicting results being reported with adjuvant VEGFR-TKI therapy in localized high risk RCC [60]. In two RCTs, sunitinib did not prolong OS while it had a significant but limited benefit on disease free survival in one of the studies. Unfortunately, a three-fold adverse event rate influencing some aspects of quality of life resulted in an unfavorable harms-benefits ratio for adjuvant VEGFR-TKI therapy. Although further trials evaluating VEGFR-TKIs in adjuvant 
setting are ongoing, it is unlikely that they will be practice changing after assessment of their contribution to value-based health care. Until data from ongoing phase III trials in the metastatic setting report significant improvement in OS, it is likely that the current adverse event profile of combined immune checkpoint inhibition and VEGF-targeted therapy prohibits their long-term administration in adjuvant studies.

\section{Conclusions and outlook}

Long-lasting remission, manageable toxicity and a synergistic effect with VEGF-targeted therapy make immune checkpoint inhibitors attractive candidates for combination therapy with antiangiogenic compounds. Clinical trials with novel checkpoint inhibitors are initiated and first results from phase I/II trials of checkpoint inhibitors with VEGFR-TKI and VEGF-monoclonal antibodies are promising. No less than 4 phase III trials are ongoing to investigate immune checkpoint inhibitors in combination with VEGF-targeted therapy in patients with treatment naïve $\mathrm{mRCC}$. These trials are designed to identify patient subgroups for appropriate treatment selection but further studies will be needed to establish markers of resistance to therapy, dosing, optimal timing, and sequencing. In parallel, neoadjuvant and presurgical studies are ongoing and will investigate whether these combinations are effective in this setting, which, in turn, may provide a rationale for adjuvant studies in patients with non-metastatic RCC with high-risk of recurrence. In addition, novel checkpoint inhibitors that ought to be less toxic are investigated actively.

Financial disclosure: The authors declare that they have no relevant financial interests.

Acknowledgements: We would like to thank the European Urological Scholarship Programme for financially supporting the research fellowship of Teele Kuusk and Nikolaos Grivas at Antoni van Leeuwenhoek Hospital/ the Netherlands Cancer Institute in Amsterdam, the Netherlands.

Conflict of interest: Axel Bex took part in advisory boards of Pfizer, Novartis, Ipsen, Eisai and Roche. He is the PI of the EORTC SURTIME trial sponsored in part by a grant from Pfizer to the EORTC.

Laurence Albiges has received consulting and advisory fees from BMS, Pfizer, Novartis, Sanofi, Amgen, Bristol-Myers Squibb, Bayer, and Cerulean; and research funding from Pfizer and Novartis.

Bernard Escudier has received fees for serving on advisory boards from Pfizer, Novartis, BristolMyers Squibb, Exelixis, and Roche and lecture fees from Pfizer and Novartis. 
John Haanen has consulted or has an advisory role for MSD Oncology, Pfizer, and Bristol-Myers Squibb, as well as gained research funding by MSD and Bristol-Myers Squibb.

Thomas Powles is a company consultant for Novartis, Pfizer, GSK, has received company speaker honoraria from Novartis, Pfizer, GSK, Genentech, performed trial participation for GSK, Pfizer, BMS, Genentech, Genetech, and received grants/research support from GSK, Pfizer, and Novartis.

The other authors have no conflict of interest to declare.

\section{References:}

1. Znaor A, Lortet-Tieulent J, Laversanne M, Jemal A, Bray F (2015) International variations and trends in renal cell carcinoma incidence and mortality. Eur Urol 67(3):519-30

2. Siegel RL, Miller KD, Jemal A. Cancer statistics, 2016 (2016) CA Cancer J Clin 66:7-30

3. Ferlay J, Steliarova-Foucher E, Lortet-Tieulent J et al. Cancer incidence and mortality patterns in Europe: estimates for 40 countries in 2012. (2013) Eur J Cancer 49: 1374-1403

4. Ljungberg B, Bensalah K, Canfield S, et al (2015) EAU guidelines on RCC-an update 2014. Eur Urol 67(5):913-24.

5. Escudier B, Porta C, Schmidinger M, et al (2016) Renal cell carcinoma: ESMO Clinical Practice Guidelines for diagnosis, treatment and follow-up. Ann Oncol 27( 5):58-68

6. Latif F, Tory K, Gnarra J, et al (1993) Identification of the von Hippel-Lindau disease tumor suppressor gene. Science 260:1317-20.

7. Linehan WM, Pinto PA, Srinivasan R, et al (2007) Identification of the genes for kidney cancer: opportunity for disease-specific targeted therapeutics. Clin Cancer Res 15;13:671-9

8. Braren V, Taylor JN, Pace W, et al (1974) Regression of metastatic renal carcinoma following nephrectomy. Urology 3:777-8

9. Fisher RI, Rosenberg SA, Fyfe G, et al (2000) Long-term survival update for high-dose recombinant interleukin-2 in patients with renal cell carcinoma. Cancer J Sci Am 6;1:55-7

10. Escudier B, Pluzanska A, Koralewski P, et al (2007) Bevacizumab plus interferon alfa-2a for treatment of metastatic renal cell carcinoma: a randomised, double-blind phase III trial. Lancet 22:370;2103-11

11. Kandalaft LE, Motz GT, Busch J, Coukos G (2011) Angiogenesis and the tumor vasculature as antitumor immune modulators: the role of vascular endothelial growth factor and endothelin. Curr Top Microbiol Immunol 344:129-48

12. Mikucki ME, Fisher DT, Matsuzaki J, et al (2015) Non-redundant Requirement for CXCR3 Signaling during Tumoricidal T Cell Trafficking across Tumor Vascular Checkpoints Nat Commun:6; 7458 
13. Hodi FS, Mihm MC, Soiffer RJ, et al (2003) Biologic activity of cytotoxic T lymphocyte-associated antigen 4 antibody blockade in previously vaccinated metastatic melanoma and ovarian carcinoma patients. Proc Natl Acad Sci U S A 15:100(8);4712-7

14. Jayson GC, Kerbel R, Ellis LM, Harris AL (2016) Antiangiogenic therapy in oncology: current status and future directions. Lancet 30:388(10043);518-29

15. Griffioen AW, Mans LA, de Graaf AM, et al (2012) Rapid angiogenesis onset after discontinuation of sunitinib treatment of renal cell carcinoma patients Clin Cancer Res 15;18(14):3961-71

16. Yang JC, Haworth L, Sherry RM, et al (2003) A randomized trial of bevacizumab, an anti-vascular endothelial growth factor antibody, for metastatic renal cancer. N Engl J Med 349:427-34

17. Motzer RJ, Hutson TE, Tomczak P, et al (2007) Sunitinib versus interferon alfa in metastatic renal-cell carcinoma. N Engl J Med 356:115-24

18. Motzer RJ, Escudier B, Oudard S, et al (2008) Efficacy of everolimus in advanced renal cell carcinoma: a double-blind, randomised, placebo-controlled phase III trial. Lancet 372:449-56

19. Escudier B, Eisen T, Stadler WM, et al (2007) Sorafenib in advanced clear-cell renal-cell carcinoma. N Engl J Med 356:125-34

20. Motzer RJ, Hutson TE, Cella D, et al (2013) Pazopanib versus sunitinib in metastatic renal-cell carcinoma. N Engl J Med 369:722-31

21. Rini Bl, Escudier B, Tomczak P, et al (2011) Comparative effectiveness of axitinib versus sorafenib in advanced renal cell carcinoma (AXIS): a randomised phase 3 trial. Lancet 378:1931-9

22. Schreiber RD, Old L, Smyth MJ (2011) Cancer Immunoediting: Integrating Immunity's Roles in Cancer Suppression and Promotion. Science 331(6024): 1565-70

23. Topalian SL, Drake CG, Pardoll DM (2015) Immune checkpoint blockade: a common denominator approach to cancer therapy. Cancer Cell 13:27(4);450-61.

24. Kirkwood JM, Butterfield LH, Tarhini AA, et al (2012) Immunotherapy of cancer in 2012. CA Cancer J Clin 62(5):309-35

25. Pardoll DM (2012) The blockade of immune checkpoints in cancer immunotherapy. Nature reviews Cancer 12(4):252- 64

26. Brahmer JR, Tykodi SS, Chow LQ, et al (2012) Safety and activity of anti-PD-L1 antibody in patients with advanced cancer. N Engl J Med 366:2455-65

27. Motzer RJ, Escudier B, McDermott DF, et al (2015) Nivolumab versus Everolimus in Advanced Renal-Cell Carcinoma. N Engl J Med 373:1803-13

28. Tartour E, Pere H, Maillere B, et al (2011) Angiogenesis and immunity: a bidirectional link potentially relevant for the monitoring of antiangiogenic therapy and the development of novel therapeutic combination with immunotherapy. Cancer Metastasis Rev 30(1):83-95 
29. Vanneman M, Dranoff G (2012) Combining Immunotherapy and Targeted Therapies in Cancer Treatment. Nat Rev Cancer 22:12(4);237-51

30. Guislain A, Gadiot J, Kaiser A et al (2015) Sunitinib pretreatment improves tumor-infiltrating lymphocyte expansion by reduction in intratumoral content of myeloid-derived suppressor cells in human renal cell carcinoma. Cancer Immunol Immunother 2015. Cancer Immunol Immunother 64(10):1241-50

31. Liu XD, Hoang A, Zhou L, et al (2015) Resistance to Antiangiogenic Therapy Is Associated with an Immunosuppressive Tumor Microenvironment in Metastatic Renal Cell Carcinoma. Cancer Immunol Res. 3(9):1017-29

32. Wallin JJ, Bendell JC, Funke R, et al (2016) Atezolizumab in combination with bevacizumab enhances antigen-specific T-cell migration in metastatic renal cell carcinoma. Nat Commun 30:7;12624 doi: 10.1038/ncomms12624

33. Amin A, Plimack ER, Infante JR, et al (2014) Nivolumab (anti-PD-1; BMS-936558, ONO-4538) in combination with sunitinib or pazopanib in patients (pts) with metastatic renal cell carcinoma (mRCC). J Clin Oncol 32(5):Abstract5010 doi:10.1200/JCO.2013.54.6911

34. Dudek AZ, Sica RA, Sidani A, et al (2016) Phase lb study of pembrolizumab in combination with bevacizumab for the treatment of metastatic renal cell carcinoma: Big Ten Cancer Research Consortium BTCRC-GU14-003. J Clin Oncol 34(2S):559

35. ClinicalTrials.gov (NCT02298959) Pembrolizumab and Ziv-aflibercept in Treating Patients With Advanced Solid Tumors.

36. M. Taylor, C.E. Dutcus, E. Schmidt, et al (2016) A phase $1 \mathrm{~b}$ trial of lenvatinib (LEN) plus pembrolizumab (PEM) in patients with selected solid tumors. Annals of Oncology 27(6): 26695

37. Clinicaltrials.gov (NCT02811861) Lenvatinib/Everolimus or Lenvatinib/Pembrolizumab Versus Sunitinib Alone as Treatment of Advanced Renal Cell Carcinoma

38. McDermott DF, Infante JR, Chowdhury S, et al (2015) A phase I/II study to assess the safety and efficacy of pazopanib (paz) and pembrolizumab (pembro) in patients (pts) with untreated advanced renal cell carcinoma (aRCC). Eur J Cancer 51:519-20

39. Atkins MB, Plimack ER, Puzanov I, et al ( 2016) Axitinib in combination with pembrolizumab in patients (pts) with advanced renal cell carcinoma (aRCC): Preliminary safety and efficacy results. Annals of Oncology 27(6):266-95

40. Larkin JMG, Gordon MS, Thistlethwaite F, et al (2016) Avelumab (MSB0010718C; anti-PD-L1) in combination with axitinib as first-line treatment for patients with advanced renal cell carcinoma. J Clin Oncol 34(Abstract TPS4580)

41. Clinicaltrials.gov ( KEYNOTE-426 NCT02853331) Study to Evaluate the Efficacy and Safety of Pembrolizumab (MK-3475) in Combination With Axitinib Versus Sunitinib Monotherapy in Participants With Renal Cell Carcinoma (MK-3475-426/KEYNOTE-426) 
42. Clinicaltrials.gov (JAVELIN Renal 101 NCT02684006) A Study of Avelumab With Axitinib Versus Sunitinib In Advanced Renal Cell Cancer (JAVELIN Renal 101)

43. Sznol M, McDermott DF, Fields JS, et al (2015) Phase lb evaluation of MPDL3280A (anti-PDL1) in combination with bevacizumab (bev) in patients (pts) with metastatic renal cell carcinoma (mRCC). J Clin Oncol 33(7):410

44. McDermott DF, Atkins MB, Motzer RJ, et al (2017) A phase II study of atezolizumab (atezo) with or without bevacizumab (bev) versus sunitinib (sun) in untreated metastatic renal cell carcinoma (mRCC) patients (pts). Paper presented at: 2017 Genitourinary Cancers Symposium; February 16-18, 2017; Orlando, FL.

45. Clinicaltrials.gov (IMmotion 151 NCT02420821) A Study of Atezolizumab in Combination With Bevacizumab Versus Sunitinib in Participants With Untreated Advanced Renal Cell Carcinoma

46. Rini Bl, Stein M, Shannon P, et al (2011) Phase 1 dose-escalation trial of tremelimumab plus sunitinib in patients with metastatic renal cell carcinoma. Cancer 117:758-67

47. ClinicalTrials.gov (NCT02724878) Study of Atezolizumab + Bevacizumab in Patients With Advanced Non-Clear Cell Renal Cell Carcinoma

48. ClinicalTrials.gov (NCT02819596) MEDI4736 Combinations in Metastatic Renal Cell Carcinoma (CALYPSO)

49. Apolo A, Mortazavi A, Stein M, et al (2017) A phase I study of cabozantinib plus nivolumab (CaboNivo) and ipilimumab (CaboNivolpi) in patients (pts) with refractory metastatic urothelial carcinoma (mUC) and other genitourinary (GU) tumors. J Clin Oncol 35, 2017 (suppl 6S; abstract 293)

50. Greef B, Eisen T (2016) Medical treatment of renal cancer: new horizons. Br J Cancer 115:50516

51. Liu J, Blake SJ, Yong MC et al (2016) Improved Efficacy of Neoadjuvant Compared to Adjuvant Immunotherapy to Eradicate Metastatic Disease. Cancer Discov 6:1382-99

52. ClinicalTrials.gov (NCT02212730) A Study Evaluating the Effect of Pembrolizumab (MK-3475) in Participants With Renal Cell Cancer (MK-3475-031)

53. ClinicalTrials.gov (NCT02575222) Study of Neoadjuvant Nivolumab in Patients With Nonmetastatic Stage II-IV Clear Cell Renal Cell Carcinoma

54. ClinicalTrials.gov (NCT02446860) A Study of Anti-PD1 (Nivolumab) Therapy as Pre- and Postoperative Therapy in Metastatic Renal Cell Cancer (ADAPTeR) (ADAPTeR)

55. ClinicalTrials.gov ( NCT02210117) Nivolumab vs Nivolumab + Bevacizumab vs Nivolumab + Ipilimumab in Metastatic Renal Cell Carcinoma (mRCC)

56. Massari F, Santoni M, Ciccarese C et al (2015) PD-1 blockade therapy in renal cell carcinoma: current studies and future promises. Cancer Treat Rev 41:114-21

57. Kasenda B, Larkin J, Gore M (2015) Immunotherapies in Early and Advanced Renal Cell Cancer. Prog Tumor Res 42:1-10 
58. Patel DN, Figlin RA, Kim HL (2016) Adjuvant Treatment for Renal Cell Carcinoma: Do We Finally Have a Major Breakthrough? Clin Adv Hematol Oncol 14(11):907-14

59. ClinicalTrials.gov (NCT03024996) A Study of Atezolizumab as Adjuvant Therapy in Participants With Renal Cell Carcinoma (RCC) at High Risk of Developing Metastasis Following Nephrectomy (IMmotion010)

60. Bex A, Albiges L, Ljungberg B, et al (2016) Updated European Association of Urology Guidelines Regarding Adjuvant Therapy for Renal Cell Carcinoma. Eur Urol doi: 10.1016/j.eururo.2016.11.034 
Table 1. Phase III studies with immune checkpoint inhibitors in combination with VEGF-targeted therapy for patients with treatment naive $\mathrm{mRCC}$

\begin{tabular}{|c|c|c|c|c|}
\hline Study & $\mathrm{N}$ & Therapy & Endpoint & Subtype \\
\hline $\begin{array}{l}\text { KEYNOTE-426 } \\
\text { NCTO2853331 }\end{array}$ & 840 & $\begin{array}{l}\text { Pembrolizumab } 200 \text { mg iv } \\
\text { every } 3 \text { weeks + axitinib } 5 \\
\text { mg po twice daily vs } \\
\text { sunitinib } 50 \mathrm{mg} \text { po once } \\
\text { daily on schedule } 4 / 2\end{array}$ & $\begin{array}{l}\text { PFS central } \\
\text { review } \\
\text { OS }\end{array}$ & $\begin{array}{l}\text { clear cell } \\
\text { component with } \\
\text { or without } \\
\text { sarcomatoid } \\
\text { features }\end{array}$ \\
\hline $\begin{array}{l}\text { JAVELIN Renal } 101 \\
\text { NCT0268400642 }\end{array}$ & 583 & $\begin{array}{l}\text { Avelumab } 10 \mathrm{mg} / \mathrm{kg} \text { iv } \\
\text { every two weeks + } \\
\text { axitinib, } 5 \mathrm{mg} \text { po twice } \\
\text { daily vs sunitinib } 50 \mathrm{mg} \text { po } \\
\text { on schedule } 4 / 2\end{array}$ & PFS & $\begin{array}{l}\text { clear cell } \\
\text { component }\end{array}$ \\
\hline $\begin{array}{l}\text { IMmotion } 151 \\
\text { NCT0242082145 }\end{array}$ & 900 & $\begin{array}{l}\text { Atezolizumab } 1200 \mathrm{mg} \text { iv } \\
\text { on days } 1 \text { and } 22 \text { of each } \\
42 \text {-day + bevacizumab } 15 \\
\mathrm{mg} / \mathrm{kg} \text { iv on days } 1 \text { and } 22 \\
\text { of each } 42 \text {-day cycle vs } \\
\text { sunitinib } 50 \mathrm{mg} \text { po on } \\
\text { schedule } 4 / 2\end{array}$ & $\begin{array}{l}\text { PFS } \\
\text { investigator } \\
\text { reviewed } \\
\text { OS in } \\
\text { participants } \\
\text { with detectable } \\
\text { PD-L1 }\end{array}$ & $\begin{array}{l}\text { clear cell } \\
\text { histology and/or } \\
\text { a component of } \\
\text { sarcomatoid } \\
\text { carcinoma }\end{array}$ \\
\hline NCT02811861 & 735 & $\begin{array}{l}\text { Lenvatinib } 18 \mathrm{mg} \text { po }+ \\
\text { everolimus } 5 \mathrm{mg} \text { po or } \\
\text { lenvatinib } 20 \mathrm{mg} \text { po+ } \\
\text { pembrolizumab } 200 \mathrm{mg} \text { iv } \\
\text { every } 3 \text { weeks vs sunitinib } \\
50 \mathrm{mg} \text { po on a schedule } \\
4 / 2\end{array}$ & PFS & $\begin{array}{l}\text { clear-cell } \\
\text { component }\end{array}$ \\
\hline
\end{tabular}


Table 2. Phase $\mathrm{I} / \mathrm{Il}$ studies with immune checkpoint inhibitors in combination with VEGF-targeted therapy with response rate

\begin{tabular}{|c|c|c|c|c|c|c|c|c|c|}
\hline Trial & $\begin{array}{l}\text { Study name, } \\
\text { setting }\end{array}$ & $\begin{array}{l}\text { Study name, } \\
\text { combination (dose) }\end{array}$ & $\begin{array}{l}\text { Nr of } \\
\text { patients } \\
\text { (evaluable } \\
\text { for } \\
\text { response/all) }\end{array}$ & ORR nr,\% & CR $n r, \%$ & PR nr,\% & SD nr,\% & PD nr, \% & $\begin{array}{l}\text { mPFS } \\
\text { months }\end{array}$ \\
\hline $\begin{array}{l}\text { Phase I, } \\
\text { NCT01472081* }\end{array}$ & $\begin{array}{l}\text { Checkmate } \\
016, \mathrm{mRCC}, 1 \mathrm{st} \\
\text { line }^{33}\end{array}$ & $\begin{array}{l}\text { nivolumab } 5 \mathrm{mg} / \mathrm{kg} \text { q3w } \\
\text { +sunitinib } 50 \mathrm{mg}\end{array}$ & 33 & $17(52 \%)$ & $1(3 \%)$ & $16(48 \%)$ & $10(30 \%)$ & $1(3 \%)$ & 48.9 \\
\hline $\begin{array}{l}\text { Phase I, } \\
\text { NCT01472081* }\end{array}$ & $\begin{array}{l}\text { Checkmate } \\
016, \mathrm{mRCC}, 1 \text { st } \\
\text { line }^{33}\end{array}$ & $\begin{array}{l}\text { nivolumab2 mg/kg q3w+ } \\
\text { pazopanib } 800 \mathrm{mg}\end{array}$ & 20 & $9(45 \%)$ & 0 & $9(45 \%)$ & $7(35 \%)$ & $4(20 \%)$ & 31.4 \\
\hline $\begin{array}{l}\text { Phase I, } \\
\text { NCT02496208* }\end{array}$ & $\begin{array}{l}\text { mRCC, 2nd } \\
\text { line } 49\end{array}$ & $\begin{array}{l}\text { nivolumab } 3 \mathrm{mg} / \mathrm{kg} \\
\mathrm{q} 2 \mathrm{w}+\text { cabozantinib } 40 \mathrm{mg}\end{array}$ & $\begin{array}{l}38 / 40(3 \\
\mathrm{mRCC})\end{array}$ & $12(32 \%)$ & $1(5 \%)$ & $\begin{array}{l}11 \\
(29 \%) 1 \mathrm{mRCC}\end{array}$ & $20(53 \%)$ & NA & NA \\
\hline $\begin{array}{l}\text { Phase I, } \\
\text { NCT00372853 }\end{array}$ & $\begin{array}{l}\text { mRCC,1st or } \\
\text { 2nd line }\end{array}$ & $\begin{array}{l}\text { tremelimumab } 6-15 \\
\text { mg/kg q12w+ sunitinib } \\
37.5 \text { or } 50 \mathrm{mg}\end{array}$ & $21 / 28$ & $16(76 \%)$ & NA & $9(43 \%)$ & $7(33 \%)$ & NA & NA \\
\hline $\begin{array}{l}\text { Phase Ib, } \\
\text { NCT02348008, } \\
\text { BTCRG-GU14-003 }\end{array}$ & $\begin{array}{l}\text { mRCC, 2nd } \\
\text { line }\end{array}$ & $\begin{array}{l}\text { pembrolizumab } 200 \mathrm{mg} \\
\text { q3w+ bevacizumab } 10 \text { or } \\
15 \mathrm{mg} / \mathrm{kg}\end{array}$ & $14 / 16$ & NA & NA & $10(71 \%)$ & NA & $4(29 \%)$ & NA \\
\hline $\begin{array}{l}\text { Phase Ib/II, } \\
\text { NCT02501096 }\end{array}$ & $\begin{array}{l}\text { mRCC, 2nd } \\
\text { line }\end{array}$ & $\begin{array}{l}\text { pembrolizumab } 200 \text { mg } \\
\text { q3w+lenvatinib } 24 \text { or } 20 \\
\text { mg }\end{array}$ & $13(8 \mathrm{mRCC})$ & $9(69 \%)$ & 0 & $7(54 \%)$ & $6(46 \%)$ & 0 & NA \\
\hline
\end{tabular}




\begin{tabular}{|c|c|c|c|c|c|c|c|c|c|}
\hline $\begin{array}{l}\text { Phase I/II, } \\
\text { NCT02014636 }\end{array}$ & $\begin{array}{l}\text { Keynote-018, } \\
\text { mRCC, 1st } \\
\text { line }^{38}\end{array}$ & $\begin{array}{l}\text { pembrolizumab } 2 \mathrm{mg} / \mathrm{kg} \\
\text { q2w+pazopanib } 600 \text { or } \\
800 \mathrm{mg}\end{array}$ & 20 & $\begin{array}{l}60 \% \text { for } 800 \\
\mathrm{mg}, 20 \% \text { for } \\
600 \mathrm{mg}\end{array}$ & $1(5 \%)$ & NA & NA & NA & NA \\
\hline $\begin{array}{l}\text { Phase Ib, } \\
\text { NCT02133742 }\end{array}$ & $\begin{array}{l}\mathrm{mRCC}, 1 s t \\
\text { line }^{40}\end{array}$ & $\begin{array}{l}\text { pembrolizumab } 2 \mathrm{mg} / \mathrm{kg} \\
\text { q3w + axitinib } 5 \mathrm{mg}\end{array}$ & 52 & $37(71 \%)$ & $3(6 \%)$ & $34(65 \%)$ & $10(19 \%)$ & $2(3.8 \%)$ & 15.1 \\
\hline $\begin{array}{l}\text { Phase Ib, } \\
\text { NCT02493751 }\end{array}$ & $\begin{array}{l}\text { JAVELIN Renal } \\
100, \text { mRCC, 1st } \\
\text { line }^{40}\end{array}$ & $\begin{array}{l}\text { avelumab } 10 \mathrm{mg} / \mathrm{kg} \\
\text { q2w+axitinib } 3 \text { or } 5 \mathrm{mg} \\
\text { twice a day }\end{array}$ & 6 & $6(100 \%)$ & 0 & $6(100 \%)$ & 0 & NA & NA \\
\hline $\begin{array}{l}\text { Phase } \\
\text { I/II,NCT01633970* }\end{array}$ & $\begin{array}{l}\text { mRCC, 1st } \\
\text { line } e^{43}\end{array}$ & $\begin{array}{l}\text { atezolizumab } 20 \text { mg/kg } \\
\text { q3w+bevacizumab } \\
15 \mathrm{mg} / \mathrm{kg} \mathrm{q3w}\end{array}$ & $10 / 12$ & $4(40 \%)$ & $1(10 \%)$ & NA & $5(50 \%)$ & NA & NA \\
\hline $\begin{array}{l}\text { Phase II, } \\
\text { NCT01984242* }\end{array}$ & $\begin{array}{l}\text { Immotion 150, } \\
\text { 1st line }\end{array}$ & $\begin{array}{l}\text { atezolizumab } 1200 \text { mg } \\
\text { q3w+bevacizumab } 15 \\
\text { mg/kg }\end{array}$ & $\begin{array}{l}305 \text { (101 in } \\
\text { combination } \\
\text { arm) }\end{array}$ & $32 \%$ & $7 \%$ & NA & NA & NA & 11.7 \\
\hline
\end{tabular}

* response rate only for VEGFR-TKI or VEGF-monoclonal antibody and checkpoint inhibitor combination arm 
Table 3. Phase I/II studies with immune checkpoint inhibitors in combination with VEGF-targeted therapy and grade 3-4 adverse events

\begin{tabular}{|c|c|c|c|c|c|c|c|c|c|c|}
\hline & $\begin{array}{l}\text { Checkmate } \\
016 \\
\text { (nivoluma } \\
\text { b+sunitini } \\
\text { b) }\end{array}$ & $\begin{array}{l}\text { Checkmate } \\
016 \\
\text { (nivolumab } \\
\text { +pazopanib } \\
\text { ) }^{33}\end{array}$ & $\begin{array}{l}\text { nivolumab } \\
\text { +cabozanti } \\
\text { nib } 49\end{array}$ & $\begin{array}{l}\text { tremelimum } \\
\text { ab+sunitinib } \\
46\end{array}$ & $\begin{array}{l}\text { pembrolizum } \\
\text { ab+bevacizu } \\
\text { mab }^{34}\end{array}$ & $\begin{array}{l}\text { pembrolizum } \\
\text { ab+lenvatini } \\
b^{36}\end{array}$ & $\begin{array}{l}\text { pembro } \\
\text { lizumab } \\
+ \\
\text { axitinib } \\
39\end{array}$ & $\begin{array}{l}\text { JAVELIN } \\
\text { Renal } \\
100 \\
\text { (avelum } \\
\text { ab+axiti } \\
\text { nib ) }\end{array}$ & $\begin{array}{l}\text { Keynote-018 } \\
\text { pembrolizu } \\
\text { mab+pazopa } \\
\text { nib }^{38}\end{array}$ & $\begin{array}{l}\text { atezolizuma } \\
\text { b+bevacizu } \\
\text { mab }^{43}\end{array}$ \\
\hline $\begin{array}{l}\text { Nr of } \\
\text { patients }\end{array}$ & 33 & 20 & $\begin{array}{l}24(3 \\
\mathrm{mRCC})\end{array}$ & 28 & 16 & 13 (8 mRCC) & 52 & 6 & 20 & 12 \\
\hline $\begin{array}{l}\text { Grade 3- } \\
4 \text { AEs }\end{array}$ & $27(82 \%)$ & $14(70 \%)$ & $7(29 \%)$ & $17(61 \%)$ & $0 \%$ & $9(69 \%)$ & $\begin{array}{l}28(53 \% \\
1\end{array}$ & $5(83 \%)$ & $13(65 \%)$ & $7(58 \%)$ \\
\hline fatigue & $9 \%$ & $15 \%$ & $2(8 \%)$ & 1 & & 1 & $6 \%$ & & & \\
\hline \multicolumn{11}{|l|}{$\begin{array}{l}\text { nausea, } \\
\text { vomiting }\end{array}$} \\
\hline artralgia & & & & & & 1 & & & & \\
\hline $\begin{array}{l}\text { hyperten } \\
\text { sion }\end{array}$ & $18 \%$ & $10 \%$ & & & & 1 & $17 \%$ & $33 \%$ & & 3 \\
\hline $\begin{array}{l}\text { hand- } \\
\text { foot } \\
\text { syndrom } \\
\text { e }\end{array}$ & & & & & & & & $17 \%$ & & \\
\hline $\begin{array}{l}\text { mucositi } \\
\mathrm{s}\end{array}$ & & & & 1 & & & & $17 \%$ & & \\
\hline \multicolumn{11}{|l|}{$\begin{array}{l}\text { pneumo } \\
\text { nitis }\end{array}$} \\
\hline $\begin{array}{l}\text { aseptic } \\
\text { meningit } \\
\text { is }\end{array}$ & & & $1 / 40$ & & & & & & & \\
\hline colitis & & & $1 / 40$ & & & & & & & \\
\hline $\begin{array}{l}\text { elevated } \\
\text { ALT }\end{array}$ & $18 \%$ & $20 \%$ & & & & & $6 \%$ & & & \\
\hline
\end{tabular}




\begin{tabular}{|c|c|c|c|c|c|c|c|c|c|}
\hline $\begin{array}{l}\text { elevated } \\
\text { AST }\end{array}$ & $9 \%$ & $20 \%$ & & & & & & & \\
\hline $\begin{array}{l}\text { elevated } \\
\text { ALT/AST }\end{array}$ & & & & & 1 & & & $13(65 \%)$ & \\
\hline $\begin{array}{l}\text { elevatio } \\
\mathrm{n} \text { of } \\
\text { lipase }\end{array}$ & & & $3(13 \%)$ & & & & $17 \%$ & & \\
\hline $\begin{array}{l}\text { hypercal } \\
\text { cemia }\end{array}$ & & & & & & & & & 1 \\
\hline $\begin{array}{l}\text { hyperuri } \\
\text { cemia }\end{array}$ & & & & & & & & & \\
\hline $\begin{array}{l}\text { hypopho } \\
\text { sphatem } \\
\text { ia }\end{array}$ & & & $4(17 \%)$ & & & & & & \\
\hline $\begin{array}{l}\text { hyponat } \\
\text { riemia }\end{array}$ & $15 \%$ & & $4(17 \%)$ & & 1 & & & & \\
\hline $\begin{array}{l}\text { lymphoc } \\
\text { ytopenia }\end{array}$ & $15 \%$ & & & & & & & & \\
\hline $\begin{array}{l}\text { neutrop } \\
\text { enia }\end{array}$ & & & & & & & & & \\
\hline $\begin{array}{l}\text { proteinu } \\
\text { ria }\end{array}$ & & & & & & & $17 \%$ & & \\
\hline diarrhea & $9 \%$ & $20 \%$ & $2(8 \%)$ & & 1 & $10 \%$ & & & \\
\hline $\begin{array}{l}\text { renal } \\
\text { insufficie } \\
\text { ncy }\end{array}$ & & & & 2 & & & & & \\
\hline $\begin{array}{l}\text { respirato } \\
\text { ry } \\
\text { insufficie } \\
\text { ncy }\end{array}$ & & & & & & & & & 1 \\
\hline $\begin{array}{l}\text { dyspnoe } \\
\text { a }\end{array}$ & & & & 1 & & & & & \\
\hline
\end{tabular}




\begin{tabular}{|c|c|c|c|}
\hline $\begin{array}{l}\text { headach } \\
\text { e }\end{array}$ & & $8 \%$ & \\
\hline $\begin{array}{l}\text { tumour } \\
\text { pain }\end{array}$ & & & 1 \\
\hline $\begin{array}{l}\text { postoper } \\
\text { ative } \\
\text { wound } \\
\text { infection }\end{array}$ & & & 1 \\
\hline death & 1 & & \\
\hline $\begin{array}{l}\text { weight } \\
\text { loss }\end{array}$ & & $6 \%$ & \\
\hline
\end{tabular}


Figure 1. Synergistic effect of VEGFR-and checkpoint inhibitors

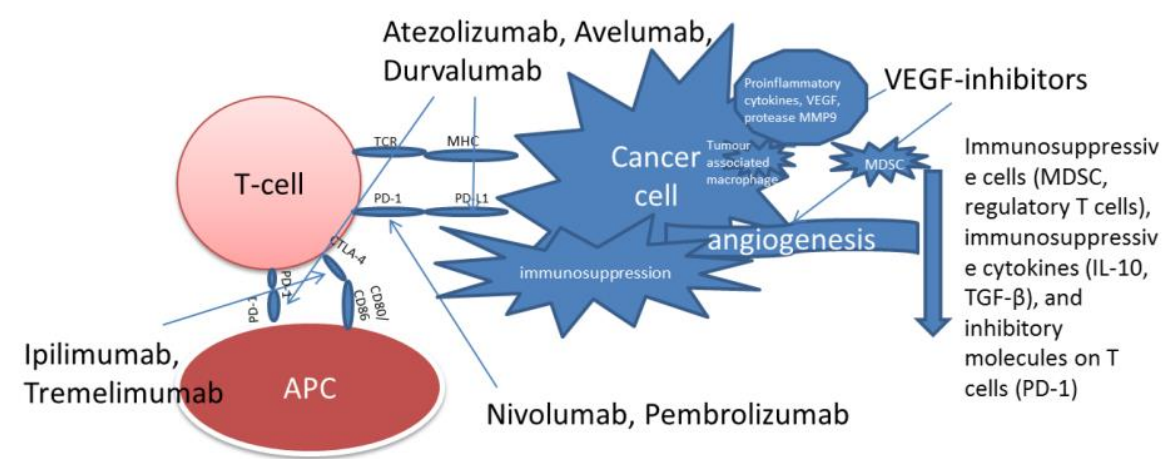

Synergistic effect of VEGF-and checkpoint inhibitors 


\section{CHAPTER 2}

Lymphatic drainage from renal tumors in vivo: a prospective sentinel node study using SPECT/CT imaging

Teele Kuusk, Roderick De Bruijn , Oscar R. Brouwer , Jeroen De Jong , Maarten Donswijk , Nikolaos Grivas, Kees Hendricksen, Simon Horenblas , Warner Prevoo, Renato A. Valdés Olmos, Henk G. Van Der Poel , Bas WG. Van Rhijn , Esther M. Wit , Axel Bex

J Urol. 2018 Jun;199(6):1426-1432. 


\section{ABSTRACT}

Purpose: Lymphatic drainage from renal tumors is unpredictable and in vivo drainage studies of primary lymphatic landing sites may reveal the variability and dynamics of lymphatic connections. The purpose of this study was to investigate the lymphatic drainage pattern from renal tumors in vivo with SPECT/CT imaging after intra-tumoral radiotracer injection.

Materials and methods: We conducted a phase II prospective single-arm study to investigate the distribution of SNs from renal tumors on SPECT/CT imaging. Patients with cT1-3 $(<10 \mathrm{~cm})$ cNOMO renal tumors of any subtype were enrolled. After intra-tumoral ultrasound guided injection of $0.4 \mathrm{ml}$ 99mTc-nanocolloid, preoperative imaging of SNs with lymphoscintigraphy and SPECT/CT was performed. SN and locoregional non-SNs were resected using a gamma probe in combination with a mobile gamma camera. The primary study endpoint was location of SNs outside the locoregional retroperitoneal templates (LRT) on SPECT/CT imaging. Using a Simon Minimax two-stage design to detect a $25 \%$ extra-LRT location of SNs on imaging with an alpha of 0.05 and a power of $80 \%$, at least 40 patients with SN imaging on SPECT/CT were needed.

Results: Sixty-eight patients were included. Forty patients had preoperative SPECT/CT imaging of SNs and were used for primary endpoint analysis. Lymphatic drainage outside the LRT was observed in 14 (35\%) patients. Eight patients (20\%) had supradiaphragmatic SN.

Conclusions: SNs from renal tumors were mainly located in their respective LRT, but simultaneous SNs located outside the suggested LND templates, including supradiaphragmatic SNs were observed in more than one third of the patients. 


\section{Introduction}

The role of lymph node dissection (LND) in the management of renal cell carcinoma (RCC) is still under debate. The randomized European Organisation for the Research and Treatment of Cancer (EORTC) 30881 trial and several retrospective studies including a propensity score-based analysis did not reveal a survival benefit in favour of LND in patients with clinically non-metastatic disease $[1,2]$. Thus, guidelines do not routinely recommend LND for clinically localized RCC. On the other hand, some retrospective studies suggest that in high-risk patients with early occult lymph node metastasis LND may provide durable long-term survival and potentially cure $[3,4,5,6,7,8]$. This has fuelled the debate whether LND should be performed in non-metastatic high-risk tumors in whom the incidence of occult lymph node metastasis may be higher than the $4 \%$ reported in the randomized EORTC trial [1]. Not only may LND provide a survival benefit in a patient population which was underrepresented in the EORTC trial, it may also improve local staging. With the advent of several adjuvant studies investigating immune checkpoint inhibition, proper staging of high-risk disease is gaining importance. However, the quality of evidence for LND in this patient population is poor and retrospective data is biased by heterogeneity in patient populations, disease stage and surgical templates [4]. Not surprisingly, conflicting conclusions regarding a potential benefit of LND are found in the extensive literature while others recommend LND for staging purposes in tumors with high risk features [3]. Clearly, a better understanding of the basics of lymphatic drainage from renal tumors is required before embarking on further clinical LND studies investigating patient outcome or prognosis [9]. Cadaveric dye dissection studies as well as autopsy and in vivo mapping studies in patients with kidney cancer have revealed the major anatomical regions of potential drainage. However, the location of the first lymph nodes receiving direct drainage from individual tumors, the lymphovascular connections of renal tumors and the frequency of simultaneous or isolated supradiaphragmatic drainage have not been studied comprehensively in vivo. Previously, we and others have reported the feasibility $[10,11,12]$ of sentinel node (SN) imaging from renal tumors in humans. Here, we report results from a prospective phase II imaging study to describe lymphatic drainage pattern in patients with renal tumors using dynamic lymphoscintigraphy and SPECT/CT with prespecified endpoints.

\section{Materials and Methods}

\section{Patients}

From 2008-2017, 68 patients were enrolled in a prospective phase II study to investigate lymphatic drainage and distribution of SNs in renal tumors (NL26406.031.08; registered at www.ccmo.nl). The ethics committee approved the study, and all patients signed written informed consent. Inclusion criteria were cT1-3 renal tumors $\leq 10 \mathrm{~cm}$ of any subtype, clinically and radiographically nonmetastatic disease (cNOMO), age $\geq 18$ years, life expectancy $>3$ months, WHO performance status 0-1 and no prior systemic therapy. Primary endpoint was the percentage of SNs located at any site outside the left or right locoregional retroperitoneal template (LRT), as defined below, on lymphoscintigraphy and subsequent SPECT/CT imaging. Secondary endpoints included the percentage of SNs with occult metastases and the false-negative rate. 
Definition of the boundaries of the locoregional retroperitoneal template (LRT)

Currently, there is no consensus on the retroperitoneal surgical LND template for renal tumors [6]. For the purpose of this study and based on previous anatomical studies $[9,13]$, the LRT for tumors on the right side was defined to include right renal hilar, paracaval, retrocaval, precaval and interaortocaval LNs from the upper margin of the crus of the diaphragm down to the right common iliac artery crossing the inferior vena cava (supplementary figure 1). For tumors from the left kidney, the LRT included left renal hilar, paraaortic, retroaortic, and preaortic LNs from the level of the crus to the bifurcation of the aorta $[9,13]$ (supplementary figure 2 ).

\section{Sentinel node imaging}

Based on the feasibility study [10], a dose of $225 \mathrm{MBq}$ of $99 \mathrm{mTc}$-nanocolloid in a volume of $0.4 \mathrm{ml}$ was percutaneously injected under ultrasound guidance into the tumor one day before surgery. Tumors $\leq 10 \mathrm{~cm}$ were chosen to guarantee a homogenous intratumoral radiotracer distribution. Primary tumors of $\leq 4 \mathrm{~cm}$ or $4-10 \mathrm{~cm}$ were injected with one or 2-4 depots of $0.4 \mathrm{ml}$ respectively, avoiding necrotic areas. Potential loss of the tracer to the bloodstream during the injection was monitored with a gamma camera. After 20 minutes (early dynamic), and 2-4 hours (late static) anterior, posterior and lateral planar lymphoscintigraphy of the affected site was performed. SPECT and low dose CT was acquired and fused 4 hours after injection (SymbiaT, Siemens, Erlangen, Germany). Anatomical location of the SNs was determined by multiplanar imaging reconstruction (Osirix Dicom viewer, Pixmeo, Geneva, Switzerland). SNs were defined as LNs draining directly from the tumor on planar dynamic lymphoscintigraphy. SPECT/CT was used to image their anatomical location. In case of multiple LNs at lymphoscintigraphy, the nodes appearing in the early dynamic phase in the LRT and if applicable - simultaneously in basins outside the LRT, were considered to be SNs (supplementary figure 3). The following day, the primary tumor and SN were resected. The surgical approach (open, laparoscopic, robot-assisted) depended on primary tumor complexity. At surgery, SN(s) were located by preoperative SPECT/CT images, a gamma-probe (Neoprobe, Johnson\&Johnson Medical, Hamburg, Germany) and a portable gamma camera (Sentinella, S102,GEM imaging, Valencia, Spain). After SN excision, the portable gamma camera was used to verify complete SN removal. For ethical reasons, only SNs accessible through the chosen surgical approach were removed. Subsequently, LND of the ipsilateral LRT was performed to study the false-negative rate. All harvested LNs were measured ex vivo with gamma probe and camera to determine radioactive count rates.

\section{Statistical Analysis}

A Simon's Two-Stage Minimax design was used to detect that $25 \%$ of SNs receiving drainage from renal tumors on imaging with SPECT/CT are located outside the respective LRT as defined above with an alpha of 0.05 and a power of $80 \%$. This design allowed early termination of the study in the first stage, if after 22 patients location outside the LRT is a rare event (defined as $f 10 \%$ ). The study was extended into the second stage and 40 patients with SN imaging on SPECT/CT were included for 
analysis of the primary endpoint. Further, descriptive statistics were used (SPSS Inc software, version 22.0, Chicago, IL). Confidence intervals ( $\mathrm{Cl}$ ) for proportions were reported as $95 \% \mathrm{CI}$.

\section{Results}

Primary endpoint:

Lymphoscintigraphy and SPECT/CT visualized at least one SN in 59\% (40/68) of patients $(95 \% \mathrm{Cl}$ : 46.9-69.7). Excluding technical and inclusion criteria errors, the non-visualization rate was $26 \%$ (95\% $\mathrm{Cl}$ : 17.5-38.0) (flow-chart 1, supplementary flow-chart 1 and 2). Patient characteristics are shown in table 1. The 40 patients with SN imaging on SPECT/CT were used for primary endpoint analysis. Most of the tumors were RCC, with a median size of $6 \mathrm{~cm}$ (IQR 4.7-7.5 cm). A total of 63 (median 1, IQR 1-2) SNs appeared on imaging. Thirty-four patients had successful intraoperative sampling of their infradiaphragmatic SNs, which correlated with the SPECT/CT location. Reasons for not sampling the SN were no activity detected with the gamma-probe or camera despite imaging on SPECT/CT (5 patients), or inaccessible infradiaphragmatic location (1 patient). Conversely, in $6 / 28$ patients who had non-visualization on imaging, radioactive LNs were detected with the gammaprobe and subsequently harvested. These patients were not included in the primary endpoint analysis. Based on imaging, 37 patients had at least one $S N$ in the respective LRT and 26 patients (65\%, 95\% Cl: 49.5-77.8) had SNs exclusively within the LRT. The drainage from tumors on the right side was predominantly into interaortocaval and retrocaval SNs (figure 1) and from the left mainly into paraaortic SNs (figure 2).

On the right side, 6 of 18 patients (33\%, 95\% Cl:16-56) with SNs had simultaneous drainage to interaortocaval, retrocaval, left preaortic or paraaortic and left supraclavicular lymph nodes. Only 3 patients with right-sided tumors had SNs in the right paracaval and renal hilar region and none had drainage to precaval LNs (figure 1 ).

Regarding drainage from the left side, only 3 patients had direct left renal hilar SNs. Nine of 22 patients (41\%, 95\% Cl: 23.2-61.2) with SN from left-sided tumors had simultaneous renal hilar, mediastinal, left supraclavicular, retrocrural, left common iliac, renal fossa and interaortocaval SNs (figure 2).

In total, 14 patients (35\%, 95\% Cl: $22.1-50.5)$ had SNs outside their respective LRT, of whom 8 (20\%, $\mathrm{Cl}: 10.5-34.7)$ had supradiaphragmatic SNs. No association was found in relation to intrarenal tumor location, size, grade or subtype.

Secondary endpoints:

Only 1 of 40 patients $(2.5 \%, 95 \% \mathrm{Cl}: 0.4-12.8)$ had an occult SN metastasis from a papillary type II pT1b RCC. The non-SN in the LRT were free of disease. None of the other patients had SN or LN metastases in their respective LRT. An analysis of the false-negative rate was not meaningful.

\section{Discussion}

LN metastasis in RCC is a poor prognostic factor for overall and disease specific survival $[4,7]$. Several reasons can be identified for the controversial survival data after LND. Due to the low incidence of occult LN metastasis in the prospective EORTC study the trial was clearly underpowered to detect a 
survival benefit [1]. Other arguments include early concomitant distant metastasis, either through haematogenic or lymphatic spread. Aberrant drainage to LN located outside the conventional surgical templates may be responsible for the latter.

The hypothesis of a widely variable lymphatic drainage from RCC is supported by earlier studies in patients with pathological node positive disease demonstrating a range of anatomical locations of LN metastases from RCC $[14,15,16]$. According to available data and in concordance with our study, the location of tumor draining LNs can be unpredictable [17].

To date, this is the largest prospective study investigating the location and pattern of primary lymphatic drainage from renal tumors following intratumoral radiotracer injection in vivo. An intriguing pattern emerging from this study is that $20 \%$ of the renal tumors drain supradiaphragmatically, in addition to retroperitoneal LNs. This finding supports direct drainage through the thoracic duct (TD) to the lungs and the mediastinum, which are among the most common distant metastatic sites in RCC $[6,18,19,20,21]$. It has been hypothesized that pulmonary metastases may be a consequence of direct lymphatic drainage into the subclavian vein and subsequent vascular spread into the lungs, which drain into the mediastinal nodes [20,22]. In addition, in an autopsy study [18] only $26 \%$ of the patients with established LN metastases had positive retroperitoneal LNs, whereas $65 \%$ of all LN metastases were mediastinal. Another study concerning 1828 autopsy records described a broad variation of metastatic LN locations [14]. Ipsilateral renal hilar LN metastases were found in only $7 \%$ of the patients. The highest percentage of LN metastases was mediastinal in $66 \%$, retroperitoneal in $36 \%$, paraaortic in $26 \%$ and supraclavicular in $20 \%$. However, all patients had multiple lymphatic metastases, precluding the conclusion that the first draining LN had been located in one of these supradiaphragmatic locations. Others have reported $22 \%$ of all LN metastases located in the mediastinum, while cases with supraclavicular and isolated contralateral iliac LN metastases have also been described $[15,16,17]$. In conjunction with these data, our study suggests that lymphatic drainage from renal tumors connects to major blood vessels by the lymphovenous connection of the TD. The TD has a higher intraluminal pressure than the venous system and has valves, that prevent lymphatic flow straight to the mediastinum and thoracic organs [23]. Another study group using peripheral renal tumor radiotracer injection did not perform supradiaphragmatic imaging and therefore did not observe this drainage pattern [11]. In RCC, it is often believed that the draining retroperitoneal LNs are located in the hilar region branching off into the paracaval, interaortocaval or paraaortic basins depending on the laterality of the renal tumor $[6,24,25]$. However, our study showed drainage in $35 \%$ of the tumors $(95 \% \mathrm{Cl}$ : 22.1 50.5 ) to SNs located outside the suggested LRTs (supplementary figures 1 and 2). This would have resulted in missing a substantial percentage of primary landing sites, if LND were performed within the limits of these templates $[24,25]$. Our findings of aberrant $S N$ regions reveal that lymphatic drainage from renal tumors exhibits an individual variability. Accordingly, generalized preconceived LND templates will not include primary LN draining sites in all patients. This study has a number of limitations. Currently, the SN procedure has no clinical implication and the high percentage of nonvisualization may limit its applicability for RCC. Direct drainage into the TD without any intervening LN has been described in human cadaver studies injecting blue dye20 and may be a potential explanation for the relatively high number of non-visualization of SNs in our study; however, this assumption cannot be confirmed since the resolution of the portable gamma camera is not suited to monitor lymphatic drainage in real time in deeper body parts. Theoretically, nonvisualization may also occur due to an absent connection of the tumor to lymphatic vessels or due to 
primary haematogenic outflow [22]. The Swedish group studying peripheral tumor radiotracer injection did not specifically report non-visualization; however, they succeeded to image SNs in only 3 of 11 patients with SPECT/CT, while the majority were detected by gamma probe only [11]. It is important to point out that detection of occult LN-metastases was not the primary endpoint of our study. Nor was the study designed to demonstrate whether the SN procedure leads to timely resection of early occult LN-micrometastases, which in turn may positively influence the course of the disease. This would have required a different design, a larger sample size and inclusion of mainly high-risk RCC patients which may have a pN1 rate of $17-44 \%$ in T2b-T3b tumors [13]. To test the in vivo drainage pattern on imaging, this study predominantly included tumors of smaller size of any subtype to guarantee an even distribution of the radiotracer in the tumor. As a consequence, the majority of patients had a low or intermediate risk of recurrence and low rate of pN1 [26]. In this study only one patient with a papillary type II RCC had occult LN metastases in $2 \mathrm{SNs}$. He is diseasefree 7 years after surgery, but this outcome needs to be interpreted with caution. This single case does not reflect the possible incidence of occult $L N$ metastases in patients with high-risk tumors of other subtypes nor the potential of the SN procedure to improve diagnostic accuracy and outcome. Perhaps, an important future application of SN detection could be in translational research to elucidate the early process of lymphatic metastasis and priming of the immune system in nodes receiving the first drainage from the primary tumor. Currently, it is unknown why and how RCC spreads through the lymphatics. Recently, a group has shown that almost all resected SNs from RCC, which were non-metastatic on H\&E staining, contained single cell metastases on flow-cytometry $[27,28]$. Current sequencing techniques may characterize the genotype of these single tumor cells and the immune environment, which may greatly advance our knowledge of metastatic spread of RCC. Finally, our study is limited by the lack of harvesting and histological examination of the supradiaphragmatic SNs.

\section{Conclusions}

This prospective study of in vivo lymphatic drainage patterns from renal tumors reveals that lymphatic drainage exhibits high individual variability. In $35 \%$ of the patients additional SNs were located outside the respective LRT, including supradiaphragmatic nodes. These findings have potential implications for the design of future clinical or translational studies investigating lymphonodular involvement.

Abbreviations: lymph node (LN), lymph node dissection (LND), renal cell carcinoma (RCC), sentinel node (SN), single-photon emission computed tomography with computed tomography (SPECT/CT), locoregional retroperitoneal template (LRT)

Financial disclosure: The authors declare that they have no relevant financial interests. Conflict of interests: No conflict of interests.

Acknowledgements: We gratefully acknowledge Andre Jagt from Antoni van Leeuwenhoek Netherlands Cancer Institute for technical assistance. We would like to thank the European Urological Scholarship Programme for financially supporting the research fellowship of Teele Kuusk 
at Antoni van Leeuwenhoek Hospital/ the Netherlands Cancer Institute in Amsterdam, the Netherlands.

\section{References}

[1] Blom JHM, Van Poppel H, Maréchal JM, et al. Radical Nephrectomy with and without LymphNode Dissection: Final Results of European Organization for Research and Treatment of Cancer (EORTC) Randomized Phase 3 Trial 3088. Eur Urol 55:28,2009

[2] Gershman B, Thompson RH, Moreira DM, et al. Radical Nephrectomy With or Without Lymph Node Dissection for Nonmetastatic Renal Cell Carcinoma: A Propensity Score-based Analysis. Eur Urol 71:560,2017

[3] Brito J 3rd, Gershman B. The role of lymph node dissection in the contemporary management of renal cell carcinoma: A critical appraisal of the evidence. Urol Oncol 35:623,2017

[4] Bekema HJ, Maclennan S, Imamura M, et al. Systematic review of adrenalectomy and lymph node dissection in locally advanced renal cell carcinoma. Eur Urol 64:799,2013

[5] Schafhauser W, Ebert A, Brod J, et al. Lymph node involvement in renal cell carcinoma and survival chance by systematic lymphadenectomy. Anticancer Res 19:1573,1999

[6] Capitanio $U$, Leibovich $B$. The rationale and the role of lymph node dissection in renal cell carcinoma. World J Urol 35:497,2017

[7] Capitanio U, Suardi N, Matloob R, et al. Extent of lymph node dissection at nephrectomy affects cancer-specific survival and metastatic progression in specific sub-categories of patients with renal cell carcinoma (RCC). BJU Int 114:210,2014

[8] Gershman B, Moreira DM, Thompson RH, et al. Renal Cell Carcinoma with Isolated Lymph Node Involvement: Long-term Natural History and Predictors of Oncologic Outcomes Following Surgical Resection. Eur Urol 72:300,2017

[9] Karmali RJ, Suami H, Wood CG, et al. Lymphatic drainage in renal cell carcinoma: back to the basics. BJU Int 114:806,2014

[10] Bex A, Vermeeren L, de Windt G, et al. Feasibility of sentinel node detection in renal cell carcinoma: a pilot study. Eur J Nucl Med Mol Imaging 37:1117,2010

[11] Sherif AM, Eriksson E, Thörn M, et al. Sentinel node detection in RCC. A feasibility study for detection of tumour-draining lymph nodes. BJU Int 109:1134,2011

[12] Bex A, Vermeeren L, Meinhardt W, et al. Intraoperative sentinel node identification and sampling

in clinically node-negative renal cell carcinoma: initial experience in 20 patients. World J Urol 29:793, 2011

[13] Blute ML, Leibovich BC, Cheville JC, et al. A protocol for performing extended lymph node dissection using primary tumor pathological features for patients treated with radical nephrectomy for clear cell renal cell carcinoma. J Urol 172:465,2004

[14] Saitoh H, Nakayama M, Nakamura K, et al. Distant metastasis of renal adenocarcinoma in nephrectomized cases. J Urol 127:1092,1982

[15] Wright FW. Enlarged hilar and mediastinal nodes (and especially lower right hilar node enlargement) as a sign of metastasis of a renal tumour. Clin Radiol 28:431,1977

[16] Mahon TG, Libshitz HI. Mediastinal metastases of infradiaphragmatic malignancies. Eur J Radiol 15:130,1992 
[17] Hulten L, Rosencrantz M, Seeman T, et al. Occurrence and localization of lymph node metastases in renal carcinoma. Scand J Urol Nephrol 3:129,1969

[18] Johnsen JA, Hellsten S. Lymphatogenous spread of renal cell carcinoma: an autopsy study. J Urol 157:450,1997

[19] Assouad J, Riquet M, Berna P, et al. Intrapulmonary lymph node metastasis and renal cell carcinoma. Eur J Cardiothorac Surg 31:132,2007

[20] Assouad J, Riquet M, Foucault C, et al. Renal lymphatic drainage and thoracic duct connections: implications for cancer spread. Lymphology 39:26,2006

[21] Riquet $M$, Berna $P$, Brian $E$, et al. Intrathoracic lymph node metastases from extrathoracic carcinoma: the place for surgery. Ann Thorac Surg 88:200,2009

[22] Brouwer OR, Noe A, Olmos RA, et al. Lymphatic drainage from renal cell carcinoma along the thoracic duct visualized with SPECT/CT. Lymphat Res Biol 11:233,2013

[23] Pflug J, Calnan J. The valves of the thoracic duct at the angulus venosus. Br J Surg 55:911,1968

[24] Parker AE. Studies on the main posterior lymph channels of the abdomen and their connections with the lymphatics of the genitourinary system. Am J Anat 56:409,1935

[25] Crispen PL, Breau RH, Allmer C, et al. Lymph node dissection at the time of radical nephrectomy for high-risk clear cell RCC: indications and recommendations for surgical templates. Eur Urol 59:18,2011

[26] Leibovich BC, Blute ML, Cheville JC, et al. Prediction of progression after radical nephrectomy for patients with clear cell renal cell carcinoma: a stratification tool for prospective clinical trials. Cancer 97:1663,2003

[27] Hartana CA, Kinn J, Rosenblatt R, et al. Detection of micrometastases by flow cytometry in sentinel lymph nodes from patients with renal tumours. Br J Cancer 115:957,2016

[28] Kuusk T, Bex A. Re: Detection of Micrometastases by Flow Cytometry in Sentinel Lymph Nodes from Patients with Renal Tumours. Eur Urol 71:691,2017 
Flow chart 1.

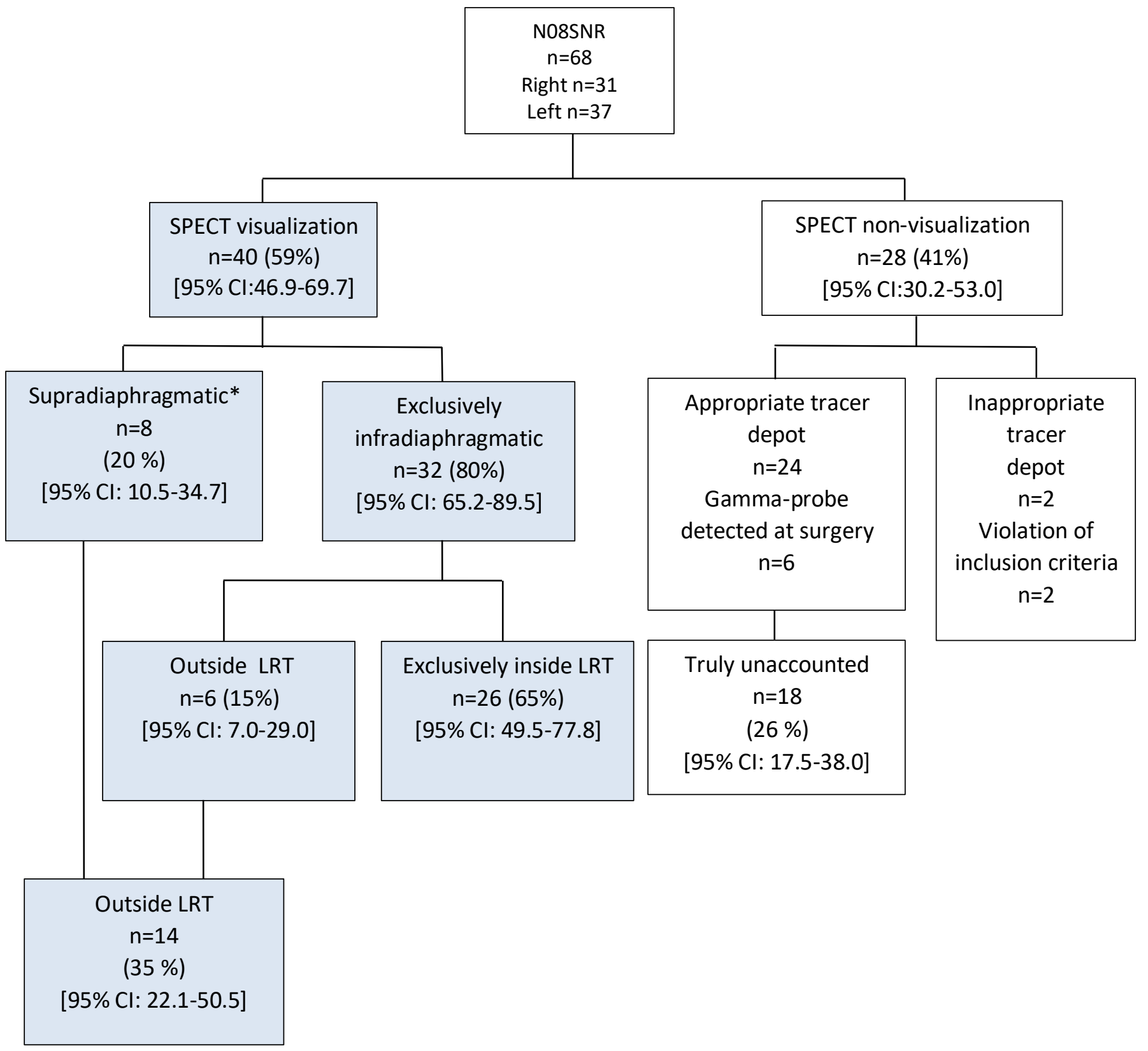

Legend: Results for all study patients. Patients with SN visualization on SPECT/CT ( $n=40$, light blue) used for analysis of the primary study endpoint. *Most of the supradiaphragmatic nodes were with a simultaneous retroperitoneal node. $\mathrm{N}=$ are number of patients with $\mathrm{SN}$ on imaging, not the number of SN. 
Table 1. Patient and tumor characteristics

\begin{tabular}{|c|c|}
\hline Number of patients & 40 \\
\hline Median age (range) & $58(38-74)$ \\
\hline Median tumour size in $\mathrm{cm}$ (range) & $6(3-10)$ \\
\hline \multicolumn{2}{|l|}{ pT stage } \\
\hline T1a & $6(14.5 \%)$ \\
\hline $\mathrm{T} 1 \mathrm{~b}$ & $21(53 \%)$ \\
\hline $\mathrm{T} 2 \mathrm{a}$ & $6(15 \%)$ \\
\hline $\mathrm{T} 2 \mathrm{~b}$ & $2(5 \%)$ \\
\hline T3a & $5(12.5 \%)$ \\
\hline \multicolumn{2}{|l|}{ pN stage } \\
\hline NO & $37(92.5 \%)$ \\
\hline N1 & $1(2.5 \%)$ \\
\hline $\mathrm{Nx}$ & $2(5 \%)$ \\
\hline Right side & $18(45 \%)$ \\
\hline Upper pole & 4 \\
\hline Interpolar & 9 \\
\hline Lower pole & 5 \\
\hline Left side & $22(55 \%)$ \\
\hline Upper pole & 5 \\
\hline Middle pole & 7 \\
\hline Lower pole & 10 \\
\hline \multicolumn{2}{|l|}{ Histology } \\
\hline Clear cell RCC & $24(60 \%)$ \\
\hline Papillary type 1 RCC & $5(12.5 \%)$ \\
\hline Papillary type 2 RCC & $3(7.5 \%)$ \\
\hline Chromophobe RCC & $4(10 \%)$ \\
\hline Oncocytoma & $3(7.5 \%)$ \\
\hline Solitary fibrous tumor & $1(2.5 \%)$ \\
\hline \multicolumn{2}{|l|}{ Leibovich score } \\
\hline Low & $9(37.5 \%)$ \\
\hline Intermediate & $11(45.8 \%)$ \\
\hline High & $4(16.6 \%)$ \\
\hline \multicolumn{2}{|l|}{ Surgical type } \\
\hline Open, radical nephrectomy & $7(17.5 \%)$ \\
\hline Open, partial nephrectomy & $16(40 \%)$ \\
\hline Laparoscopic, radical nephrectomy & $3(7.5 \%)$ \\
\hline Robotic, radical nephrectomy & $1(2.5 \%)$ \\
\hline Robotic, partial nephrectomy & $13(32.5 \%)$ \\
\hline
\end{tabular}


Figure 1.

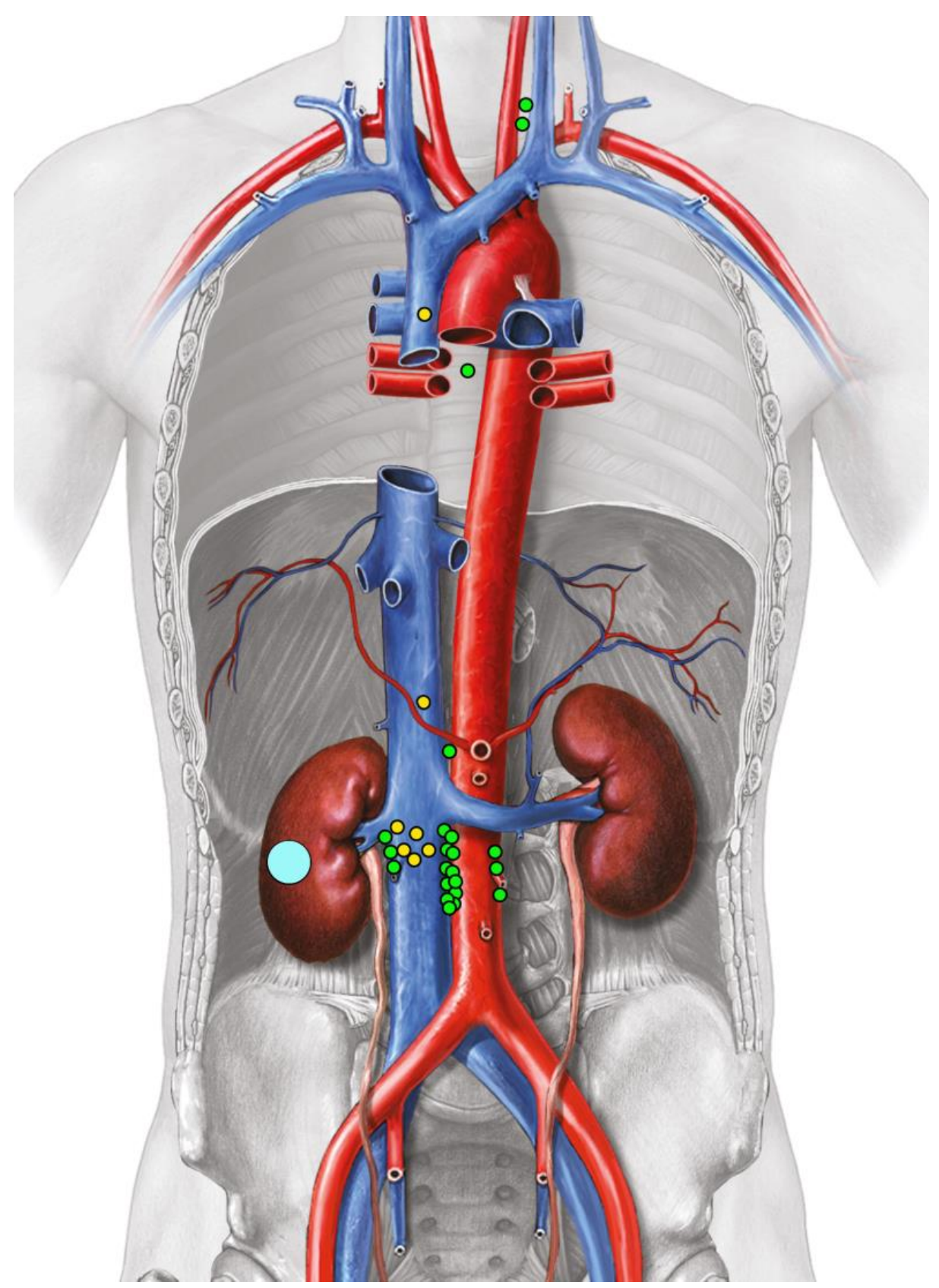

Legend: Distribution of 29 SNs from 18 right kidney tumors at SPECT/CT. Green SNs locate ventrally to blood vessels, yellow SNs dorsally. Image printed with permission of A.D.A.M. Images. 
Figure 2.

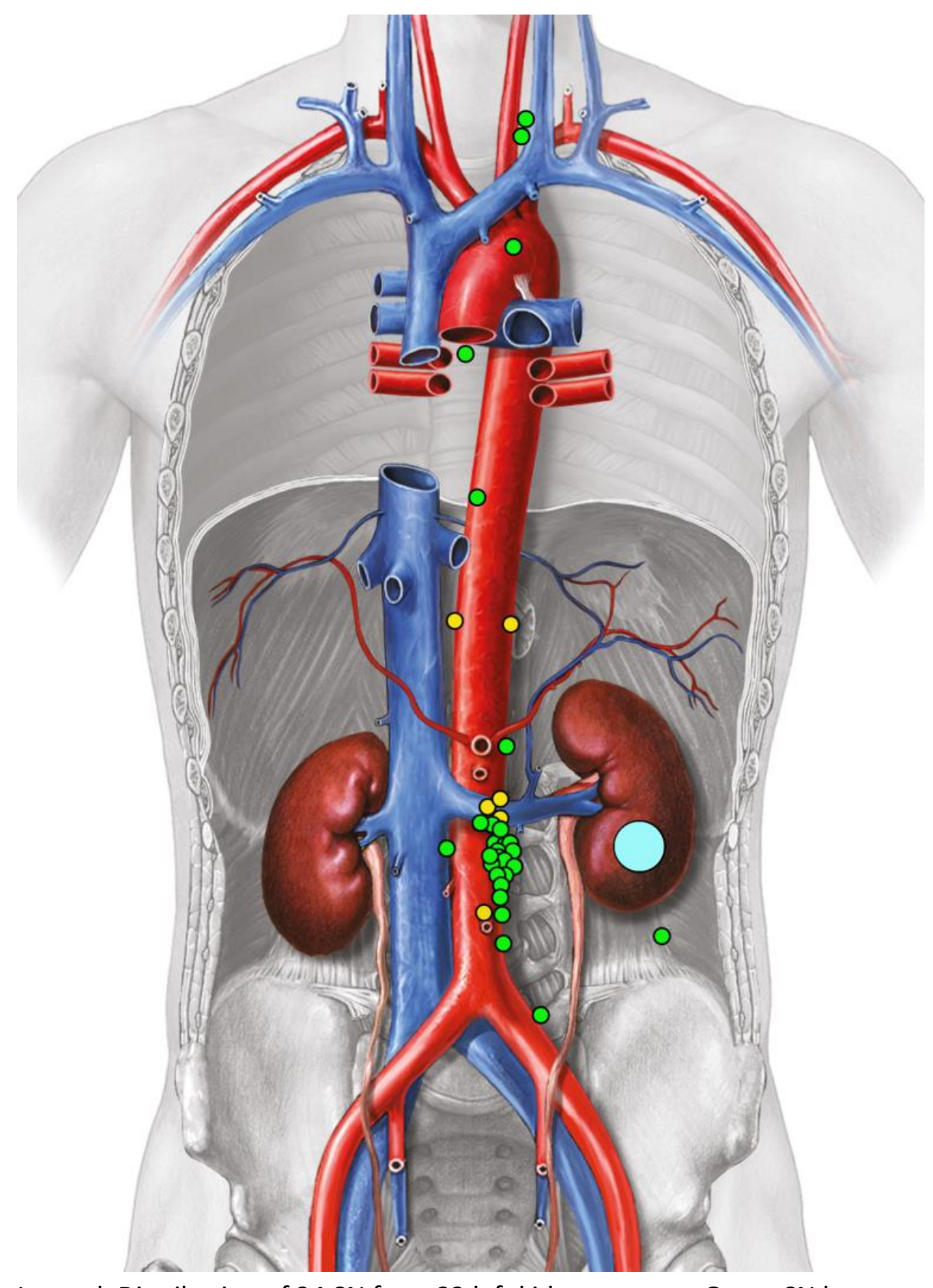

Legend: Distribution of 34 SN from 22 left kidney tumors. Green SN locate ventrally to blood vessels, yellow SN dorsally. Image printed with permission of A.D.A.M. Images. 
Supplementary Flow chart 1.

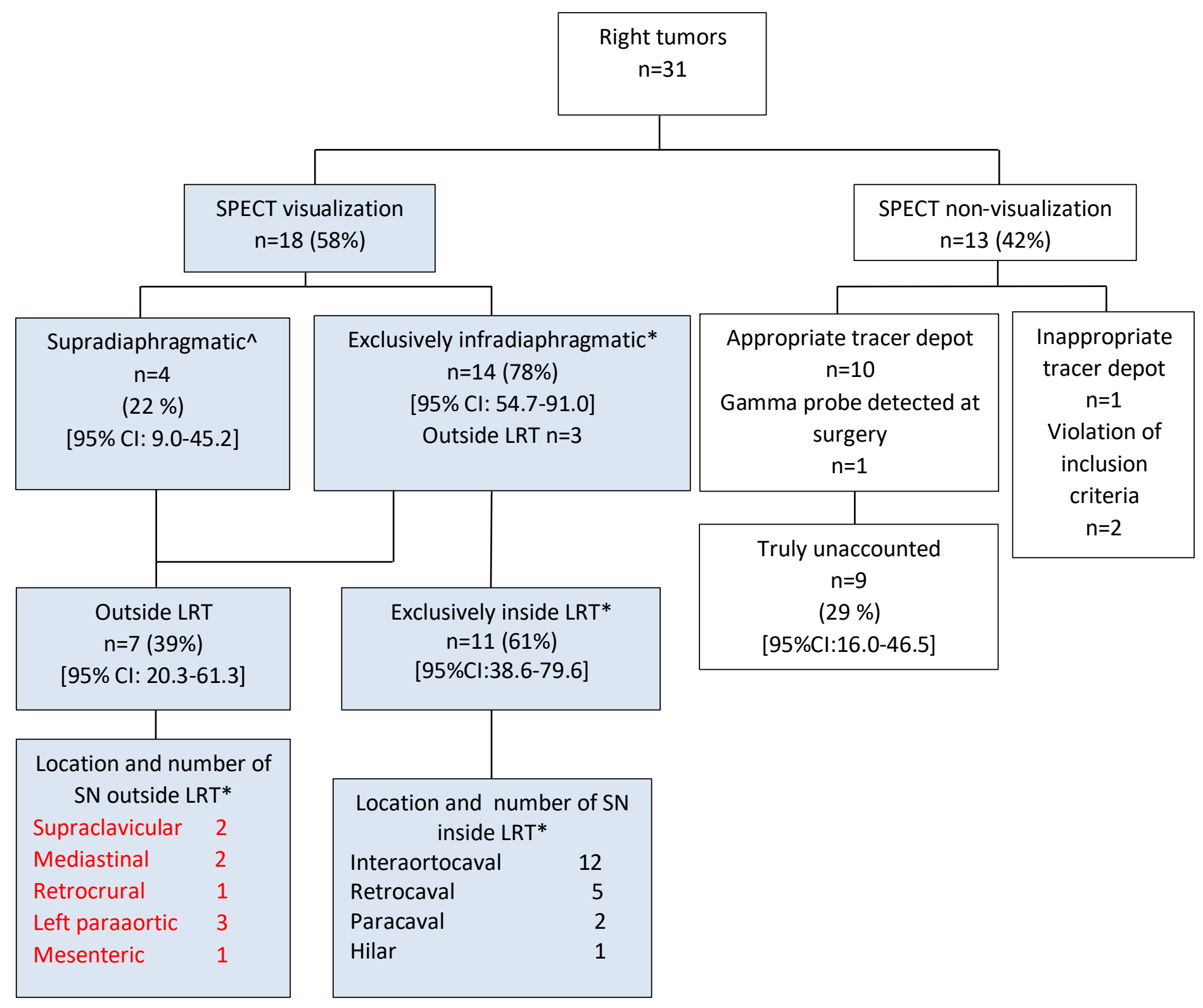

Legend: Results for right kidney tumors. Patients with SN visualization on SPECT/CT ( $n=18$, light blue) used for analysis of the primary study endpoint. $\mathrm{N}=$ are number of patients, the location of the nodes includes all individual SN. The nodes in red are outside the locoregional retroperitoneal templates (LRT).

$\wedge$ Most of the supradiaphragmatic nodes were with a simultaneous retroperitoneal node

* Several patients had up to 2 sentinel nodes 
Supplementary Flow chart 2.

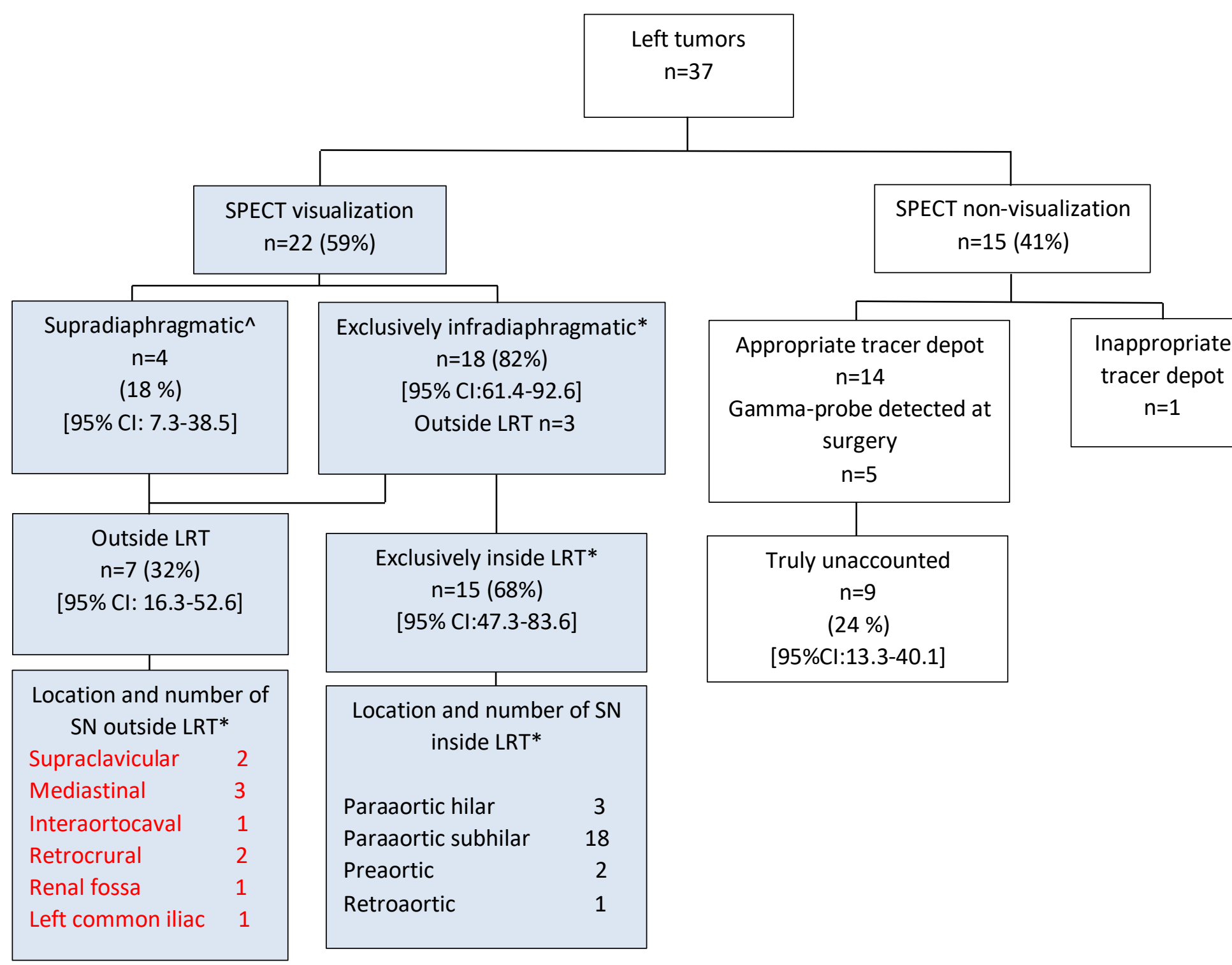

Legend: Results for left kidney tumors. Patients with SN visualization on SPECT/CT ( $n=22$, light blue) used for analysis of the primary study endpoint. $\mathrm{N}=$ are number of patients, the location of the nodes includes all individual SN. The nodes in red are outside the locoregional retroperitoneal templates (LRT).

^ Most of the supradiaphragmatic nodes were with a simultaneous retroperitoneal node

* Several patients had up to 2 sentinel nodes 
Supplementary Figure 1.

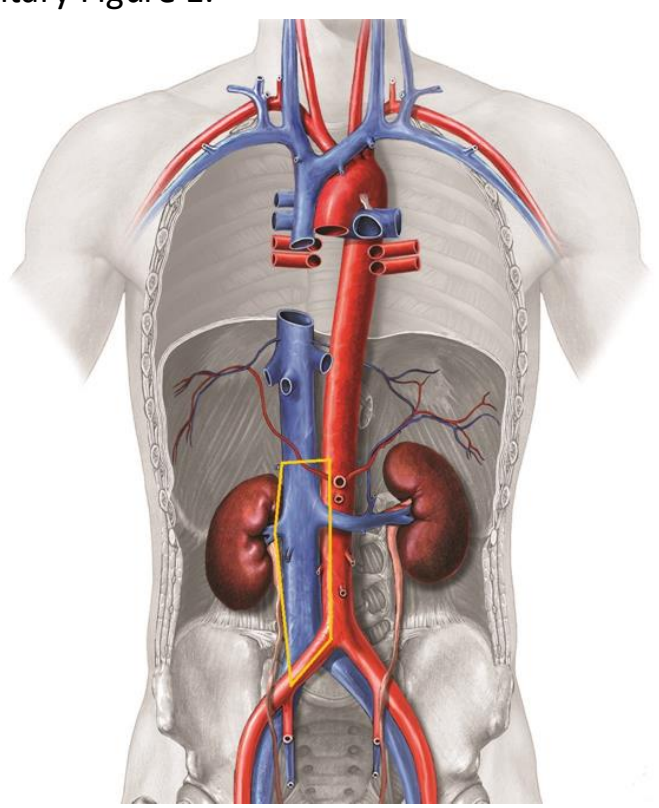

Legend: Schematic LND template for right side RCC. Image printed with permission of A.D.A.M. Images.

Supplementary Figure 2.

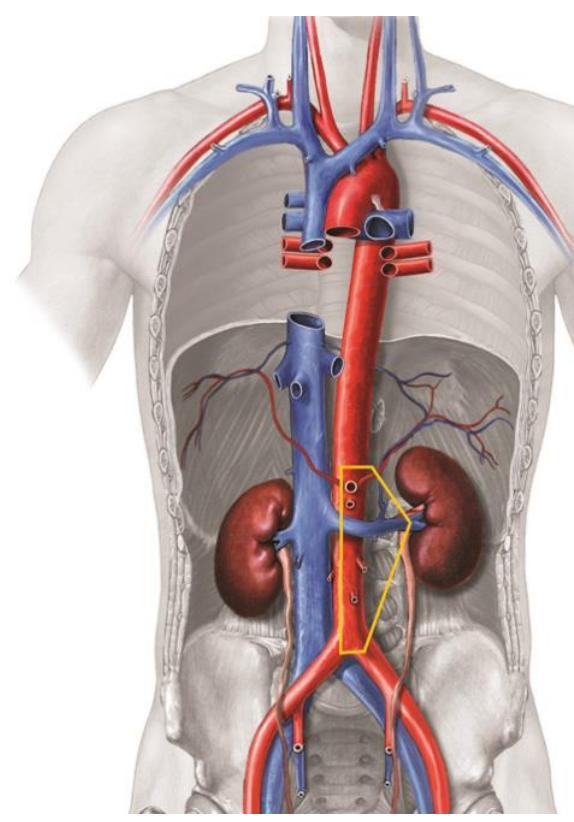

Legend: Schematic LND template for left side RCC. Image printed with permission of A.D.A.M. Images. 
Supplementary Figure 3. 3D fused SPECT and low dose CT images of mediastinal and paraaortic SNs.
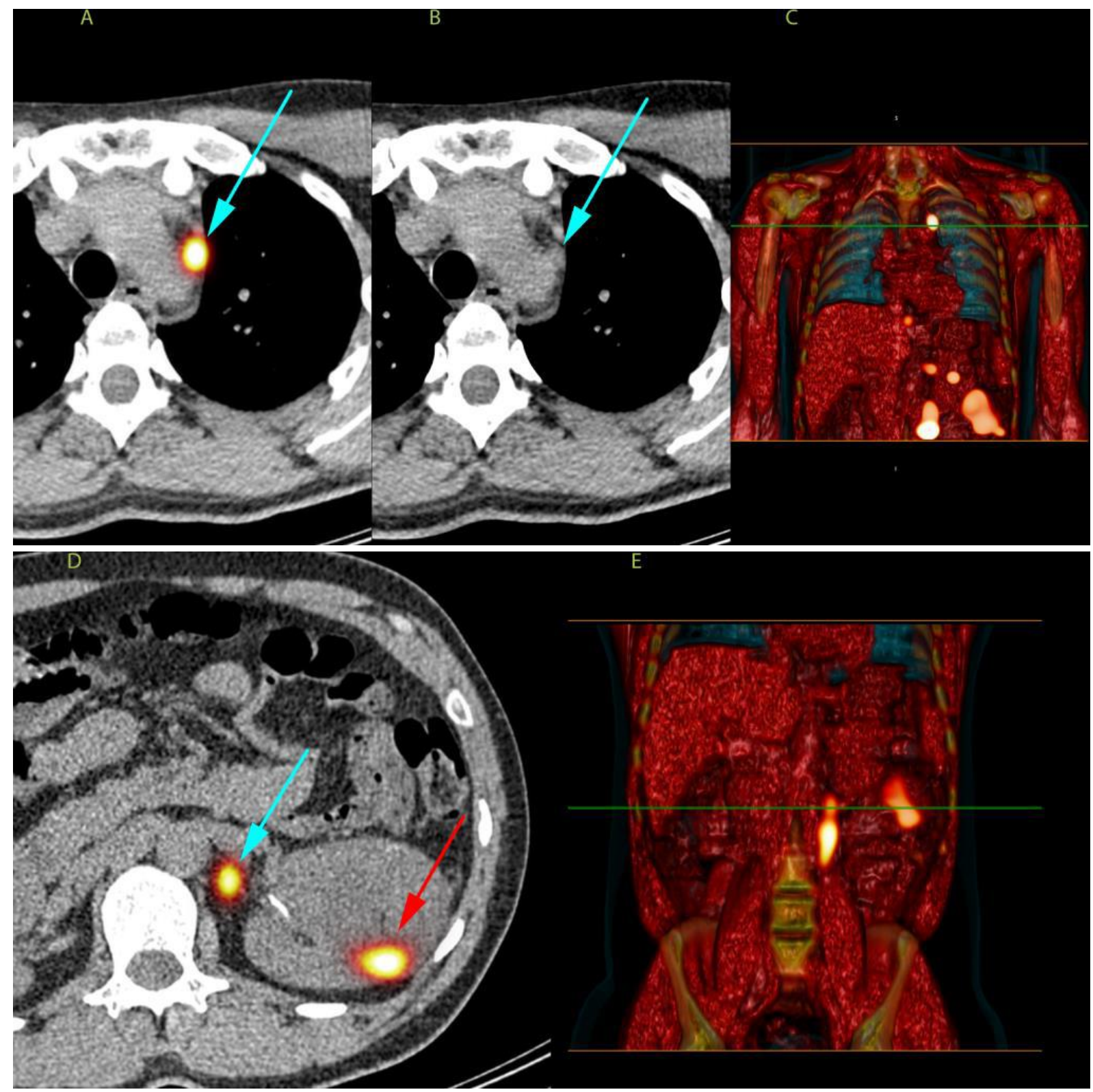

Legend: A. Axial reconstruction of fused SPECT/CT image showing the mediastinal SN (blue arrow). B. The same LN on CT (blue arrow). C. 3D volume rendering of the fused SPECT and low dose CT images showing SNs, green line grossing the mediastinal SN. D. Axial reconstruction of fused SPECT/CT image showing the paraaortic SN (blue arrow) and radioisotope depot in the tumor (red arrow) E. 3D volume rendering of the fused SPECT and low dose CT images showing a paraaortic SN (green line crossing the SN and depot in the tumor). 


\section{EDITORIAL COMMENT}

Kuusk et al report a prospective clinical trial to describe the distribution of SNs in patients with renal tumors undergoing surgery. The primary end point of this study was to identify the location of these

SNs outside the standard retroperitoneal node templates. Not surprisingly these nodes were located outside the standard retroperitoneal templates in 14 patients (35\%) in whom SNs were detected. Interestingly 8 of these 14 patients had a supradiaphragmatic SN. The authors could not find an association between SN location and tumor factors. This study reinforces the idea that lymph node drainage/metastases in kidney cancer do not follow patterns as predictable as testicular and penile cancer do. In addition, this study could explain the recent findings of prospective and retrospective studies (references 1 and 2 in study) which showed a lack of survival benefit when performing routinely lymphadenectomies during radical nephrectomy. This work suggests that even if future clinical trials of lymphadenectomy are performed in patients at very high risk for LN metastases, the results might not differ from those of EORTC 30881 (reference 1 in article). Therefore, it would be prudent to focus on subsets of patients who might benefit from surgery and, therefore, perform RPLND up front in patients who tend to have a higher rate of retroperitoneal only nodal metastases (eg papillary type $2 \mathrm{RCC}$ ) or perform salvage RPLND in patients who have retroperitoneal only nodal recurrences after nephrectomy. Additional basic/translational studies with novel imaging techniques are needed to move the field forward.

Jose A. Karam

University M. D. Anderson Cancer Center

Houston, Texas 


\section{CHAPTER 3}

Outcome of sentinel lymph node biopsy in patients with clinically non-metastatic renal cell carcinoma

Teele Kuusk, Roderick De Bruijn, Oscar R Brouwer, Jeroen De Jong, Maarten Donswijk, Kees Hendricksena, Simon Horenblas, Katarzyna Jóźwiak, Warner Prevoo, Renato A Valdés Olmos, Henk G Van Der Poel, Bas WG Van Rhijn, Esther M Wit, Axel Bex

Scand J Urol. 2018 Oct - Dec;52(5-6):411-418. 


\section{ABSTRACT}

Objective: To investigate rate of occult SN metastases, oncological outcome and association of recurrence with pattern of lymphatic tumour drainage in RCC.

Materials and Methods: A pooled RCC subgroup analysis of secondary endpoints from a published feasibility and a phase II prospective single-arm SN study to investigate oncological outcome. Patients with cT1-3 $(<10 \mathrm{~cm})$ cNOMO RCC of any subtype were enrolled. After intratumoural injection of Tc99m nanocolloid, preoperative imaging of SNs with SPECT/CT was followed by (partial) nephrectomy with SN and regional lymph node dissection using a $p$-probe. The patients were followed with a risk-adapted surveillance program. Endpoints of the studies were analyzed using Fisher's exact, Chi-square or MannWhitney $U$ tests and Cox proportional hazard models.

Results: Sixty-six RCC patients were included. Two patients (3\% [95\% Cl 0.5-11\%]) had occult SN metastases with a disease-free survival (DFS) of 57 and 72 months. Ten patients (15\% [95\% Cl 7-26\%]) developed recurrences, and 4 (6\% [95\% Cl 2.3-14.5\%]) died of disease during a median follow-up of 57 months (IQR 18-72 months). Occurrence of distant metachronous metastases were associated with tumour size $(H R=1.39, p=0.01)$, pT stage (HR=6.83, $p<0.01$ for comparison T1 vs T3/4), Leibovich score $(H R=8.42, p=0.01$ for comparison low vs high) and interaortocaval sentinel lymph node location ( $H R=10.52, p=0.03$ for comparison yes vs no).

Conclusions: The rate of occult metastatic SN is low, but long DFS was observed in two patients with occult SN metastases. We hypothesize an interaortocaval lymphatic route in thoracic recurrences. Evaluation of the prognostic and therapeutic role of sentinel lymph node biopsy (SLNB) requires a clinical trial in high-risk RCC. 


\section{Introduction}

Renal cell carcinoma (RCC) is the sixteenth cause of cancer death worldwide [1]. Most deaths from RCC are due to the metachronous dissemination of tumour cells after nephrectomy with curative intent to lung, lymph nodes (LN), and other metastatic sites [2]. For many cancers, dissemination of tumour cells through locoregional lymphatics is the most common metastatic route. However, in RCC retroperitoneal $L N$ are rarely the first metastatic site $[2,3]$ and it is believed that tumour cell dissemination is primarily hematogenic. In a randomized study comparing locoregional lymph node dissection (LND) to no LND, only $4 \%$ of clinically negative $L N$ contained occult metastases [4] and the study failed to report a survival benefit. As a consequence, guidelines do not recommend LND for clinically non-metastatic RCC. A recent systematic review supports this, although the evidence synthesis suggests that patients with early occult LN metastases may benefit from LND [5]. In addition, several adjuvant immune-checkpoint studies in RCC have renewed the interest in the prognostic value of LN positive disease to improve risk assessment of patients for trial inclusion. However, a general limitation of the utility of LND is not only an unproven survival benefit, but the overall low rate of isolated LN metastases in small renal masses which represent the majority of kidney cancer diagnosis. The therapeutic and prognostic window of LND may be very limited. LND may provide prognostic information: an increasing yield of pathologically confirmed LN metastases is associated with a high percentage of synchronous distant metastasis and poor outcome $[6,7]$. Apart from a poorly defined LND template for renal tumours, other potential reasons for the low rate of locoregional LN metastases may include a predominantly hematogenic dissemination, a low lymphangiogenic activity of clear cell RCC as well as a lymphatic drainage pattern outside proposed locoregional LND templates $[8,9,10]$. Recently, we reported the primary objective of a prospective sentinel node (SN) imaging study in renal tumours [8]. In a third of the patients, lymphatic drainage after intratumoural radiotracer injection occurred outside regional retroperitoneal LN basins while in $20 \%$ of the patients, lymphatic drainage involved additional supradiaphragmatic landing sites including lymph nodes at the terminal end of the thoracic duct (TD) [11]. Direct lymphatic drainage from 
kidneys through the TD often bypassing retroperitoneal nodes has been previously reported in cadaveric dye studies and has been postulated as the main cause of pulmonary and mediastinal metastasis [12]. Here, we report the secondary oncological objectives of the prospective phase 2 imaging study for the subgroup of patients with RCC. To increase statistical power, we performed a pooled analysis with the RCC subgroup from a previous feasibility study $[13,14]$.

\section{Patients and Methods}

From 2008-2017, 68 patients entered a phase 2 prospective single-arm study to investigate lymphatic drainage and the distribution of SNs in renal tumours (NO8SNR; registered under NL26406.031.08 at www.ccmo.nl). Primary endpoint was the percentage of SNs located at any site outside the left or right regional LND templates on lymphoscintigraphy and subsequent SPECT/CT imaging and was reported recently [8]. In this study, we analyze the secondary oncological objectives of the phase 2 study which were rate of occult LN metastasis, DFS, rate of recurrence, death of disease and a potential association of thoracic recurrence with lymphatic drainage pattern. The study had ethics committee approval and all patients signed written informed consent. Inclusion criteria were cT1-3 renal tumours $\leq 10 \mathrm{~cm}$ of any subtype, clinically and radiologically non-metastatic disease (cNOMO), age $\geq 18$ years, life expectancy $>3$ months, WHO performance status $0-1$ and no prior systemic therapy. To increase statistical power, 10 patients from a previous prospective feasibility study with identical inclusion criteria (N06SNR; registered under NL26406.031.08 at www.ccmo.nl) [13] were added for a pooled analysis of the subgroup of patients with RCC. Surgery, SN biopsy (SLNB) and imaging were performed as described earlier $[8,13]$. Briefly, one day before surgery intratumoural Tc99m-nanocolloid injection was followed by planar scintigraphy and SPECT/CT imaging. At surgery, subdiaphragmatic SNs were localized with a $\gamma$-probe and portable $\gamma$-camera. All identified SNs were resected and regional LND was performed according to a previously reported template [8]. Metachronous metastases were defined as distant metastases that have occurred anywhere at distant locations during the follow-up period. Thoracic recurrence refers to metastases anywhere in the thoracic area e.g lung, pleura, mediastinum. Surveillance was performed according to a local risk-adapted follow-up 
protocol for low-intermediate and high Leibovich risk of recurrence.

\section{Statistical analysis}

Characteristics of patients were compared between patients who did and did not develop metachronous metastases using Fisher's exact, Chi-Square or Mann-Whitney U test. Risk of metachronous metastases and thoracic recurrences was modeled with univariable Cox proportional hazard models. Multivariable analyses were not performed due to very low number of events. Kaplan-Meier survival analysis was performed to illustrate metastases-free survival curves for patients with and without interaortocaval radiotracer drainage and the two curves were compared with a log-rank test. All tests were two-sided and $p$ value $\leq 0.05$ was considered statistically significant. Statistical analyses were performed using SPSS version 22 (IBM, Chicago, IL, USA).

\section{Results}

Of 78 patients enrolled 12 patients were excluded from the analysis because of benign histology, ineligibility or incorrect radiotracer injection (table 1). Therefore, the final sample included 66 patients with predominantly ccRCC (75.8\%). The characteristics of the patients with and without distant metastases are shown in table 2. Forty-one patients $(62.1 \%)[95 \% \mathrm{Cl}$ 49-73\%] had visualization of SN on SPECT/CT imaging. On imaging, the median number of SNs was 1 (total 72, IQR 1-2). SNs were successfully harvested in 41 (62.1\%) [95\% Cl 4973\%] procedures. Twenty-seven (66\%) [95\% $\mathrm{Cl} 49-79 \%]$ patients had SNs located within the locoregional retroperitoneal basin, 14 (34\%)[95\% Cl 20-50\%] had SN outside of these regions, the remainder (25) had non-visualization on SPECT/CT imaging. Median follow-up was 57 months (IQR 18-72 months). Ten patients (15\%) [95\% Cl 7-26\%] with clear-cell RCC developed recurrences with a median DFS of 14 months (IQR 8-37). Of those, 8 patients $(80 \%[95 \% \mathrm{Cl} 44-96 \%])$ had thoracic metastases, of whom $6(60 \%)[95 \% \mathrm{Cl}$ 27-86\%] had metastases exclusively in the thoracic cavity (table 2 , specific location). Five out of 6 patients with at least one interaortocaval SN receiving radiotracer drainage from the tumour developed thoracic metastases (figure 1). Of 7 patients (10\%)[95\% $\mathrm{Cl} 4-20 \%]$ who died during follow-up, 4 (40\%) died of disease. 
Two patients (3\%) [95\% $\mathrm{Cl} 0.5-11 \%]$ had occult metastases in retroperitoneal SN. In one patient, 2 occult SN metastases without extranodal growth in a left pT1b papillary type 2 (UISS low-risk) RCC were removed which were visible on preoperative SPECT/CT imaging. In the other patient, an occult metastatic hilar SN was harvested in a right pT3a Fuhrman grade 2 (Leibovich high-risk) clear-cell RCC, which did not appear on SPECT/CT but had radiotracer activity during surgery. In both patients, template based additional LND was performed without further LN metastases and they remained free of disease. The first patient is still alive after 72 months of follow-up, the latter survived 57 months and died from another cause unrelated to RCC.

Risk of distant metastases was associated with tumour size $(H R=1.39, p=0.01)$, pT stage ( $H R=6.83, p<0.01$ for comparison T1 vs T3/4), Leibovich score (HR=8.42, $p=0.01$ for comparison low vs high) and interaortocaval $S N$ location ( $H R=10.52, p=0.03$ for comparison yes vs no) (table 2 and figure 2). Five patients out of 6 with interaortocaval SN receiving radiotracer drainage from the tumour developed thoracic metastases however, none of the factors that were considered, were associated with risk of thoracic metastases (table 3).

\section{Discussion}

This study is the first to report long-term oncological outcome of SLNB in RCC. In a predominantly low-to intermediate-risk RCC population, we found $70 \%$ of lymphatic drainage within previously proposed locoregional LND templates, whereas $30 \%$ of the tumours drained to lymphatics elsewhere and $20 \%$ supradiaphragmatically into the thoracic cavity [8]. During a long follow-up, 10 (15\%) patients developed recurrences, of which 8 (80\%) were thoracic. The occurrence of distant metastases was associated in univariate analysis with interaortocaval SN receiving lymphatic radiotracer drainage from the tumour. Two patients had occult metastases in the resected SNs with long disease-free survival (DFS). A potential reason for the low rate of SN metastases (3\%) in our cohort is the inclusion of a population with predominantly lower risk RCC. As reported previously, the selection of this population was decided upon, because the primary objective of the study was to investigate lymphatic outflow on imaging and not the clinical utility of SLNB. Although the study included patients with tumours up to $10 \mathrm{~cm}$ in diameter, larger tumours were not eligible as 
distribution of the radiotracer, lymphatic outflow and volume of the injected tracer depended on tumour size and required protocol standardization. Only $12 \%$ of patients had high-risk tumours based on Leibovich and TNM risk assessment. The randomized EORTC-trial to assess the role of LND in RCC similarly included patients of predominantly lower risk [4]. Together with retrospective studies the rate of occult LN metastasis has been reported to range between $4-7 \%$ for patients with lower risk $\operatorname{RCC}[4,15,16]$. Robust data for the incidence of isolated occult LN metastases in clinically non-metastatic high-risk patients are lacking, although a recent retrospective study on occult LN metastases in patients who took part in a randomized adjuvant trial of high-risk RCC reported occult metastases in CNO RCC as low as $2 \%$ [17]. However, in this adjuvant trial population only $3 \mathrm{LN}$ were removed on average [17]. In addition, conventional histopathology may miss limited LN metastases, and more refined protocols may be required to enhance the detection rate $[18,19]$. Although, the rate of isolated LN metastases in the literature is low, patients with LN metastases tend to have a poor prognosis while those with negative $L N$ status among other factors have better cancer specific survival (CSS) and overall survival (OS) $[5,6,7,15,16,20,21,22,23]$. The prognostic importance of $L N$ metastases remains valid in the novel Leibovich risk prediction model in which LN involvement was a predictive marker in clear-cell RCC and chromophobe RCC for progression and in clear-cell RCC also for death [24]. Of note, locoregional LN metastases have been associated with concurrent metastases in lung and liver in up to $97 \%$ of cases $[6,23]$. This suggests that a potential therapeutic and prognostic window of LND is very limited. However, the long disease-free course of the two patients in our study with isolated limited intranodal metastases despite aggressive subtypes suggests that a benefit can be achieved by resection of early occult nodal disease. This is consistent with the results of a systematic review and a retrospective study by Gershman et al, who reported on a small subset of patients with pN1MO RCC and LND who survived 5 years and derived durable CSS $[5,15]$. However, the authors also showed that most of the patients with isolated pN1 disease developed metastasis within 4 months and a 5-year CSS of only 22-39\% [15]. These contradictory results suggest that a minority of patients with early occult LN metastases can be cured if completely and timely resected, but their number is eclipsed by the far greater population of patients with concurrent subclinical distant metastases precluding any 
detectable, let alone significant impact on statistics.

The rapid systemic progression described in patients with isolated LN metastases [15] and the pattern of supradiaphragmatic drainage in our previous imaging study [8] adds to the hypothesis that in a proportion of patients, lymphatic spread of tumour cells may result in subsequent systemic metastases due to lympho-venous connections [9]. Five patients out of 6 with at least one interaortocaval SN receiving radiotracer drainage from the tumour developed thoracic metastases. This pattern supports a previously reported hypothesis that tumours with interaortocaval drainage drain straight into the TD which connects to the subclavian vein [12]. Lymphatic drainage from the kidney revealing interaortocaval connections with the thoracic cavity has been reported in early cadaver studies of lymphatic drainage from the kidney by Parker and others, who observed lymphatic drainage from the kidney to the TD without intervening $\operatorname{LN}[9,11,27]$. However, additional direct local lympho-venous connections that may cause hematogenous metastases through anastomoses between regional LN and adrenal and lumbar veins have been postulated [9].

We acknowledge that the methods applied in our study are unable to substantiate the assumption of a direct lymphatic spread into the thoracic cavity, the most common metastatic site in $\mathrm{RCC}[2,3,9,11,27]$. Of note, none of the interaortocaval SN revealed metastases using a histopathology protocol with $3 \mu \mathrm{m}$ sections of the entire node [8]. However, a recent SN study in RCC from Scandinavia using cell suspensions of the SN and flow-cytometry to detect isolated tumour cells suggests that tumour cell shedding into the lymphatics is a common process [19] and another study has demonstrated worse CSS with interaortocaval LN metastases [28]. To which extent these cells may contribute to systemic disease has not been investigated. Nevertheless, based on our findings of a supradiaphragmatic drainage pattern $[8,11]$, and the studies of others, we hypothesize that patients may develop distant metastasis through a lympho-vascular connection of the retroperitoneal nodes with the TD and the subclavian vein (figure 3). Importantly, although the TD receives drainage from mediastinal nodes, valves prevent direct flow from the duct into mediastinal lymphatics [29]. Primarily, mediastinal nodes receive their drainage from the lungs. We hypothesize that tumour cells may spread from the primary tumour through the locoregional lymphatics into the TD, sometimes without intervening retroperitoneal nodes which may in part explain the nonvisualization 
on SPECT imaging [8]. Subsequently, the tumour cells drain from the TD into

the subclavian vein and into the lungs leading to pulmonary or further mediastinal LN metastases (figure 3). Autopsy studies revealed that $66 \%$ of all LN metastases are located in the lung hilar and mediastinal nodes and not the retroperitoneal, which further supports this route [30]. This hypothesis introduces new research questions such as why some patients with RCC develop lymphogenic metastases and if early detection of micrometastases or isolated tumour cells in draining LN might be an indicator for the gatekeeper function of LNs which might prevent direct drainage to thoracic regions and hematogenous spread in these particular patients.

Our study is not without limitations. We included few high-risk patients, which may have an impact on the rate of pN1 disease. In addition, due to ethical reasons, only SN accessible through the nephrectomy approach were removed leaving supradiaphragmatic SN without pathological diagnosis. To demonstrate if the SN procedure leads to timely resection of early occult LN metastases would have required a different trial design with a larger sample size of predominantly high-risk RCC patients. Finally, the low numbers do not permit a multivariate analysis to investigate if interaortocaval drainage is a predictor for metastases independent from tumour size, pT-stage and Leibovich risk .

Our results support a future prospective SLNB study in clinically high-risk RCC patients, possibly biopsy proven with higher grade, and CNO on multi-phase $\mathrm{CT}$ imaging. The incidence of template based occult LN metastases has never been prospectively investigated in this population. Our previous imaging study suggests that $70 \%$ of all SN are located within the locoregional retroperitoneal nodes and SLNB mapping in high-risk RCC might improve the precision of LND and could potentially impact on survival in a subset of patients.

Additionally, the FDA approval of sunitinib in the adjuvant setting and 4 ongoing trials with immunotherapy require accurate risk assessment and knowledge of the LN status for staging purposes has regained importance. SLNB could potentially be of value for clinically higher risk patients in this setting.

\section{Conclusion}

The rate of occult metastatic SLNB is low, but removal resulted in long DFS. Interaortocaval 
lymphatic tumor drainage may be associated with thoracic recurrences. Evaluation of the prognostic and therapeutic role of SLNB requires a clinical trial in high-risk RCC.

Acknowledgements: We thank Professor Bradley Leibovich for his valuable comments on lymph node metastases for this work. We gratefully acknowledge Andre Jagt from Antoni van Leeuwenhoek Netherlands Cancer Institute and Camille Laurelli for technical assistance. We would like to thank the European Urological Scholarship Program for financially supporting the research fellowship of Teele Kuusk at Antoni van Leeuwenhoek Hospital/ the Netherlands Cancer Institute in Amsterdam, the Netherlands.

Financial disclosure: The authors declare that they have no relevant financial interests. Conflict of interests: No conflict of interests.

\section{References}

1. Znaor A, Lortet-Tieulent J, Laversanne M, Jemal A, Bray F. International variations and trends in renal cell carcinoma incidence and mortality. Eur Urol 2015;67(3):519-30.

2. Chandrasekar T, Klaassen Z, Goldberg H, Kulkarni GS, Hamilton RJ, Fleshner NE.

Metastatic renal cell carcinoma: Patterns and predictors of metastases-A contemporary population-based series. Urol Oncol 2017;35(11):661.e7-661.e14.

3. Bianchi M, Sun C, Jeldres $C$, et al. Distribution of metastatic sites in renal cell carcinoma: a population-based analysis. Ann Oncol 2012;23:973-80.

4. Blom JHM, Van Poppel H, Maréchal JM, et al. Radical Nephrectomy with and without Lymph-Node Dissection: Final Results of European Organization for Research and Treatment of Cancer (EORTC) Randomized Phase 3 Trial 3088. Eur Urol 2009;55:28-34.

5. Bhindi B, Wallis CJD, Boorjian SA, Thompson RH, Farrell A, Kim SP, et al. The Role of Lymph Node Dissection in the Management of Renal Cell Carcinoma: A Systematic Review and Meta-Analysis. BJU Int. 2018; 121(5):684-98.

6. Kroeger N, Pantuck AJ, Wells JC, Lawrence N, Broom R, Kim JJ, et al. Characterizing the impact of lymph node metastases on the survival outcome for metastatic renal cell carcinoma patients treated with targeted therapies. Eur Urol 2015;68(3):506-15. 
7. Pantuck AJ, Zisman A, Dorey F, Chao DH, Han KR, Said J, et al. Renal cell carcinoma with retroperitoneal lymph nodes: role of lymph node dissection. J Urol 2003;169(6):2076-83. 8. Kuusk T, De Bruijn R, Brouwer RO, De Jong J, Donswijk M, Grivas N, et al. Lymphatic drainage from renal tumors in vivo: a prospective sentinel node study using SPECT/CT imaging. J Urol. 2018;199(6):1426-32.

9. Karmali RJ, Suami H, Wood CG, Karam JA. Lymphatic drainage in renal cell carcinoma: back to the basics. BJU Int 2014;114(6):806-17.

10. Baldewijns MM, Roskams T, Ballet V, Van den Eynden GG, Van Laere SJ, Van der Auwera I, et al. A low frequency of lymph node metastasis in clear-cell renal cell carcinoma is related to low lymphangiogenic activity. BJU Int 2009;103(12):1626-31.

11. Brouwer OR, Noe A, Olmos RA, et al. Lymphatic drainage from renal cell carcinoma along the thoracic duct visualized with SPECT/CT. Lymphat Res Biol 2013;11:233-8.

12. Assouad J, Riquet M, Berna $\mathrm{P}$, et al. Intrapulmonary lymph node metastasis and renal cell carcinoma. Eur J Cardiothorac Surg 2007;31:132-4.

13. Bex A, Vermeeren $L$, de Windt $G$, et al. Feasibility of sentinel node detection in renal cell carcinoma: a pilot study. Eur J Nucl Med Mol Imaging 2010;37:1117-23.

14. Bex A, Vermeeren L, Meinhardt W, et al. Intraoperative sentinel node identification and sampling in clinically node-negative renal cell carcinoma: initial experience in 20 patients. World J Urol 2011;29:793-9.

15. Gershman B, Moreira DM, Thompson RH, Boorjian SA, Lohse CM, Costello BA, et al. Renal Cell Carcinoma with Isolated Lymph Node Involvement: Long-term Natural History and Predictors of Oncologic Outcomes Following Surgical Resection. Eur Urol 2017;72(2):300-6.

16. Gershman B, Thompson RH, Moreira DM, Boorjian SA, Tollefson MK, Lohse CM, et al. Radical Nephrectomy With or Without Lymph Node Dissection for Nonmetastatic Renal Cell Carcinoma: A Propensity Score-based Analysis. Eur Urol 2017;71(4):560-7.

17. Ristau BT, Manola J, Haas NB, Heng DYC, Messing EM, Wood CG, et al. Retroperitoneal Lymphadenectomy in High-Risk Non-Metastatic Renal Cell Carcinoma: An Analysis of the ASSURE (ECOG-ACRIN 2805) Adjuvant Trial. J Urol 2018;199(1):53-59.

18. Kiss B, Thoeny HC, Studer UE. Current Status of Lymph Node Imaging in Bladder and 
Prostate Cancer. Urology 2016;96:1-7.

19. Hartana CA, Kinn J, Rosenblatt R, Anania S, Alamdari F, Glise H, et al. Detection of micrometastases by flow cytometry in sentinel lymph nodes from patients with renal tumours. Br J Cancer 2016;11:115(8):957-66.

20. Connolly SS, Raja A, Stunell H, Parashar D, Upponi S, Warren AY, et al. Diagnostic accuracy of preoperative computed tomography used alone to detect lymph-node involvement at radical nephrectomy. Scand J Urol. 2015;49(2):142-8.

21. Blute ML, Leibovich BC, Cheville JC, et al. A protocol for performing extended lymph node dissection using primary tumor pathological features for patients treated with radical nephrectomy for clear cell renal cell carcinoma. J Urol 2004;172(2):465-9.

22. Lughezzani G, Capitanio U, Jeldres C, Isbarn H, Shariat SF, Arjane P, et al. Prognostic significance of lymph node invasion in patients with metastatic renal cell carcinoma: a population-based perspective. Cancer 2009;15;115(24):5680-7.

23. Tornberg SV, Nisen H, Visapää H, Kilpeläinen TP, Järvinen R, Mirtti T, et al. Outcome of surgery for patients with renal cell carcinoma and tumour thrombus in the era of modern targeted therapy. Scand J Urol. 2016;50(5):380-6.

24. Leibovich BC, Lohse CM, Cheville JC, Zaid HB, Boorjian SA, Frank I, et al. Predicting Oncologic Outcomes in Renal Cell Carcinoma After Surgery. Eur Urol 2018;73(5):772-780. 25. Kim K.S, Kim H.W, Kim J.C, Kwak C, Kim Y-J, Kang S.H. Predictive factor of lymph node metastases in patients with non-metastatic renal cell carcinoma; multi-center study. Eur Urol Suppl 2017;16(3);e1104.

26. Bandini M, Smith A, Zaffuto E, Pompe RS, Marchioni M, Capitanio U, et al. Effect of pathological high-risk features on cancer-specific mortality in non-metastatic clear cell renal cell carcinoma: a tool for optimizing patient selection for adjuvant therapy. World J Urol 2018;36(1):51-7.

27. Parker EA. Studies on the main posterior lymph channels of the abdomen and their connections with the lymphatics of the genito-urinary system. Am J Anat 1935;56:409-43. 28. Nini A, Larcher A, Cianflone F, Trevisani F, Terrone C, Volpe A, The Effect of Anatomical Location of Lymph Node Metastases on Cancer Specific Survival in Patients with Clear Cell Renal Cell Carcinoma. Front Surg 2018;28;5:26. 
29. Pflug J, Calnan J. The valves of the thoracic duct at the angulus venosus. Br J Surg 1968;55(12):911-6.

30. Johnsen JA, Hellsten S. Lymphatogenous spread of renal cell carcinoma: an autopsy study. J Urol 1997; 157: 450-3.

Table 1. Consort diagram for study participants

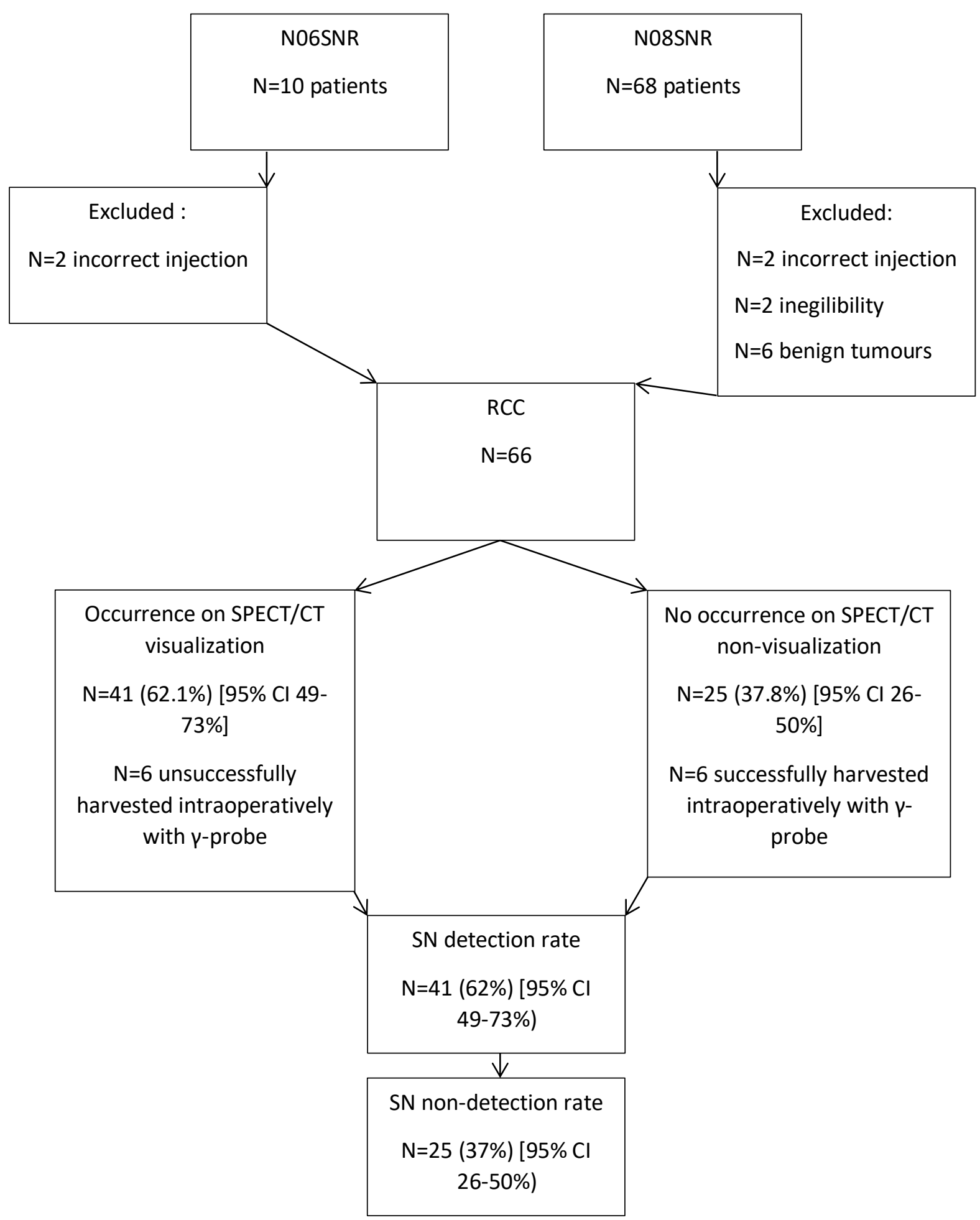


Table 2. Characteristics of patients who remained free of disease or developed metachronous metastases after SLNB

\begin{tabular}{|c|c|c|c|}
\hline & & & \\
\hline & Yes & No & $P$ value \\
\hline Number of patients & 10 & 56 & \\
\hline \multicolumn{4}{|l|}{ Gender } \\
\hline F/M & $\begin{array}{l}4(40 \%) / 6 \\
(60 \%)\end{array}$ & $\begin{array}{l}27(48 \%) / 29 \\
(52 \%)\end{array}$ & $P=0.73$ \\
\hline Age (median, IQR) & $56(52-73)$ & $59(52-64)$ & $P=0.78$ \\
\hline BMI (median, IQR) & $\begin{array}{l}25.8 \text { (24.1- } \\
32.3)\end{array}$ & $\begin{array}{l}26.7(24.1- \\
30.8)\end{array}$ & $P=0.94$ \\
\hline pT stage & & & $P=0.015$ \\
\hline T1 & $5(50 \%)$ & $44(78.6 \%)$ & \\
\hline $\mathrm{T} 2$ & $1(10 \%)$ & $9(16.1 \%)$ & \\
\hline T3 & $4(40 \%)$ & $3(5.4 \%)$ & \\
\hline pN status negative/positive & & $\begin{array}{l}54(96.4 \%) / 2 \\
(3.6 \%)\end{array}$ & NA \\
\hline Size of the tumor (median, IQR) & $\begin{array}{l}8.9(4.7- \\
10.5)\end{array}$ & 6 (IQR 5-7) & $P=0.06$ \\
\hline \multicolumn{4}{|l|}{ Location in the kidney } \\
\hline Side & & & $P=0.30$ \\
\hline Right & $7(70 \%)$ & $27(48 \%)$ & \\
\hline Left & $3(30 \%)$ & $29(52 \%)$ & \\
\hline Polarity & & & $P=0.95$ \\
\hline Upper pole & $2(20 \%)$ & $11(19.6 \%)$ & \\
\hline Intermedial pole & $4(40 \%)$ & $25(44.6 \%)$ & \\
\hline Lower pole & $4(40 \%)$ & $20(35.7 \%)$ & \\
\hline Anterior/Posterior & & & $\mathrm{P}=0.39$ \\
\hline Anterior & $5(50 \%)$ & $31(55.4 \%)$ & \\
\hline Posterior & $5(50 \%)$ & $25(44.6 \%)$ & \\
\hline \multicolumn{4}{|l|}{ Subtype } \\
\hline Clear cell RCC & $10(100 \%)$ & 40 (71.4\%) & $P=0.1$ \\
\hline Papillary type 1 RCC & & $7(12.5 \%)$ & NA \\
\hline Papillary type 2 RCC & & $3(5.4 \%)$ & NA \\
\hline Chromophobe RCC & & $5(8.9 \%)$ & NA \\
\hline NOS & & $1(1.78 \%)$ & NA \\
\hline
\end{tabular}




\begin{tabular}{|c|c|c|c|}
\hline Fuhrman grade & & & $\mathrm{P}=0.15$ \\
\hline $0 / 1$ & $1(10 \%)$ & $24(36.4 \%)$ & \\
\hline 2 & $3(30 \%)$ & $23(34.8 \%)$ & \\
\hline $3 / 4$ & $6(60 \%)$ & $19(28.7 \%)$ & \\
\hline Leibovich risk score in ccRCC & & & $\mathrm{P}=0.03$ \\
\hline Low & $2(20 \%)$ & $23(41.1 \%)$ & \\
\hline Intermediate & $4(40 \%)$ & $17(30.4 \%)$ & \\
\hline High & $4(40 \%)$ & $4(7.1 \%)$ & \\
\hline UISS risk by TNM in non-ccRCC & & & NA \\
\hline Low & & $10(17.9 \%)$ & \\
\hline Intermediate & & $2(3.6 \%)$ & \\
\hline \multicolumn{4}{|l|}{ Visualization of SN on SPECT } \\
\hline yes & $7(70 \%)$ & $34(61 \%)$ & $\mathrm{P}=0.73$ \\
\hline no & $3(30 \%)$ & $22(39 \%)$ & \\
\hline \multicolumn{4}{|l|}{ Thoracic $S N^{5}$} \\
\hline yes & $1(10 \%)$ & $6(10.7 \%)$ & $P=0.65$ \\
\hline no & $6(60 \%)$ & $28(50 \%)$ & \\
\hline Non visualization & $3(30 \%)$ & $22(39.3 \%)$ & \\
\hline \multicolumn{4}{|l|}{$\begin{array}{l}S N \text { in the locoregional lymphatic } \\
\text { drainage basin }\end{array}$} \\
\hline yes & $4(40 \%)$ & $23(41.1 \%)$ & $P=0.67$ \\
\hline no & $3(30 \%)$ & $11(19.6 \%)$ & \\
\hline Non visualization & $3(30 \%)$ & $22(39.3 \%)$ & \\
\hline \multicolumn{4}{|l|}{ IAC SN } \\
\hline yes & $6(85.7 \%)$ & $10(17.9 \%)^{\circledR}$ & $P=0.004$ \\
\hline no & $1(14.3 \%)$ & $24(42.8 \%)$ & \\
\hline Non visualization & $3(30 \%)$ & $22(39.3 \%)$ & \\
\hline $\begin{array}{l}\text { Number of SNs on imaging (median, } \\
\text { IQR) }\end{array}$ & $1(1-2)$ & $1(0-2)$ & $P=0.57$ \\
\hline $\begin{array}{l}\text { Number of harvested SNs (median, } \\
\text { IQR) }\end{array}$ & $2(1-3)$ & $1(1-3)$ & $P=0.68$ \\
\hline $\begin{array}{l}\text { Number of excised nonSNs (median, } \\
\text { IQR) }\end{array}$ & $2(1-5)$ & $1(1-4)$ & $P=0.53$ \\
\hline Site of recurrence & & & NA \\
\hline Lungs only & $3(30 \%)$ & & \\
\hline Lungs with mediastinal LN & $2(20 \%)$ & & \\
\hline Lungs and pleura & $1(10 \%)$ & & \\
\hline Retrocrural LN and mediastinal LN & $1(10 \%)$ & & \\
\hline
\end{tabular}




\begin{tabular}{llll}
\hline $\begin{array}{l}\text { RP LN* with mediastinal LN and } \\
\text { lungs }\end{array}$ & $1(10 \%)$ & & \\
\hline $\begin{array}{l}\text { Liver and nephrectomy bed } \\
\text { Contralateral kidney }\end{array}$ & $1(10 \%)$ & & \\
$\begin{array}{l}\text { Disease free survival median, IQR } \\
\text { Median follow up in months, IQR }\end{array}$ & $1(10 \%)$ & & NA \\
& $26.5 \mathrm{mth}(16-37)$ & $60 \mathrm{mth}(\mathrm{IQR}$ & $\mathrm{P}=0.58$ \\
\hline Deaths & $70)$ & $18-73)$ & \\
\hline
\end{tabular}

Legend: Results of Mann Whitney $U$ and Fisher's exact tests. P values in bold are statistically significant ( $p \leq 0.05)$. F female; M male; UISS UCLA Integrated Staging System; NA not applicable; RP retroperitoneal; SN sentinel node; RCC renal cell carcinoma; LN lymph node; IQR interquartile range; SPECT Single-photon emission computed tomography; IAC SN interaortocaval sentinel node; LND lymph node dissection. $\$$ thoracic SN located at the cervical end of the thoracic duct; * out of field recurrence in an interaortocaval LN above the renal vein after left paraaortal SN and LND in a left sided RCC. ${ }^{\circledR} 2$ out of 10 IAC SN were outside RP LND template area. 
Table 3. Association with metastases and variables

\begin{tabular}{|c|c|c|c|c|}
\hline & $\begin{array}{l}\text { Metachronous } \\
\text { metastases }\end{array}$ & & $\begin{array}{l}\text { Thoracic } \\
\text { recurrence }\end{array}$ & \\
\hline & $\mathrm{HR}[95 \% \mathrm{Cl}]$ & $P$ value & $\mathrm{HR}[95 \% \mathrm{Cl}]$ & $P$ value \\
\hline Number of events & 10 & & 8 & \\
\hline \multicolumn{5}{|l|}{ Gender } \\
\hline Male & $1.64[0.46-5.87]$ & $P=0.44$ & $1.73[0.41-7.28]$ & $P=0.46$ \\
\hline Female & 1.0 & & 1.0 & \\
\hline Age & 1.02 [0.96-1.09] & $P=0.49$ & $1.04[0.97-1.12]$ & $P=0.25$ \\
\hline BMI & $1.01[0.92-1.11]$ & $\mathrm{P}=0.82$ & 0.96 [0.85-1.10] & $P=0.57$ \\
\hline Size of the tumour & $1.39[1.06-1.83]$ & $P=0.02$ & 1.24 [0.91-1.67] & $P=0.17$ \\
\hline \multicolumn{5}{|l|}{ Tumour side } \\
\hline Right & 1.0 & & 1.0 & \\
\hline Left & $0.52[0.13-2.01]$ & $P=0.34$ & 0.39 [0.08-1.93] & $\mathrm{P}=0.25$ \\
\hline \multicolumn{5}{|l|}{ Tumour polarity } \\
\hline Upper pole & 1.0 & $P=0.84$ & 1.0 & $P=0.60$ \\
\hline Intermedial pole & $0.75[0.14-4.10]$ & $P=0.74$ & $1.17[0.12-11.25]$ & $\mathrm{P}=0.89$ \\
\hline Lower pole & $1.12[0.20-6.11]$ & $P=0.90$ & $2.26[0.25-20.24]$ & $P=0.47$ \\
\hline \multicolumn{5}{|l|}{ pT stage } \\
\hline T1 & 1.0 & & 1.0 & \\
\hline T2 & 0.94 [0.11-8.03] & $P=0.95$ & $0.95[0.11-8.12]$ & $P=0.96$ \\
\hline T3/4 & $6.83[1.83-25.51]$ & $P<0.01$ & $3.44[0.66-17.77]$ & $P=0.14$ \\
\hline \multicolumn{5}{|l|}{ Leibovich score } \\
\hline Low & 1.0 & & 1.0 & \\
\hline Intermediate & $1.94[0.35-10.59]$ & $P=0.45$ & $1.49[0.25-8.93]$ & $P=0.66$ \\
\hline High & 8.42 [1.53-46.36] & $P=0.01$ & 5.97 [0.99-36.05] & $P=0.05$ \\
\hline \multicolumn{5}{|l|}{ Subtype } \\
\hline Non-ccRCC & 1.0 & & 1.0 & \\
\hline ccRCC & $30.55[0.06-\infty]$ & $P=0.28$ & $30.86[0.03-\infty]$ & $P=0.33$ \\
\hline \multicolumn{5}{|l|}{ Fuhrman grade } \\
\hline $0 / 1$ & 1.0 & & 1.0 & \\
\hline 2 & $3.31[0.34-31.78]$ & $P=0.30$ & $1.12[0.07-17.82]$ & $P=0.94$ \\
\hline $3 / 4$ & $8.38[1.01-69.72]$ & $P=0.05$ & $8.26[0.99-68.67]$ & $P=0.05$ \\
\hline \multicolumn{5}{|l|}{ SN removed or not } \\
\hline yes & $1.21[0.31-4.68]$ & $\mathrm{P}=0.78$ & $0.87[0.21-3.62]$ & $P=0.84$ \\
\hline no & 1.0 & & 1.0 & \\
\hline Number of excised SNs & $0.96[0.77-1.20]$ & $P=0.72$ & $0.97[0.76-1.24]$ & $\mathrm{P}=0.82$ \\
\hline Number of excised nonSNs & $1.05[0.94-1.18]$ & $P=0.42$ & $0.98[0.81-1.18]$ & $P=0.83$ \\
\hline Number of SNs on imaging & $1.46[0.86-2.48]$ & $P=0.16$ & $1.53[0.87-2.69]$ & $P=0.14$ \\
\hline \multicolumn{5}{|l|}{$\begin{array}{l}\text { SNs in the locoregional } \\
\text { lymphatic drainage basin }\end{array}$} \\
\hline yes & $0.57[0.13-2.56]$ & $P=0.46$ & $0.31[0.05-1.85]$ & $P=0.20$ \\
\hline no & 1.0 & & 1.0 & \\
\hline
\end{tabular}




\begin{tabular}{lllll}
\hline Thoracic SNs & & & & \\
\hline yes & $0.75[0.09-6.27]$ & $\mathrm{P}=0.79$ & $1.16[0.13-10.41]$ & $\mathrm{P}=0.89$ \\
\hline no & 1.0 & & 1.0 & \\
$\begin{array}{l}\text { Visualization of SNs on SPECT/CT } \\
\text { yes }\end{array}$ & $1.79[0.46-6.93]$ & $\mathrm{P}=0.40$ & $1.26[0.30-5.29]$ & $\mathrm{P}=0.75$ \\
\hline no & 1.0 & & 1.0 & \\
\hline IAC SN & $10.52[1.26-87.48]$ & $\mathrm{P}=0.03$ & $139.2[0.06-\infty]$ & $\mathrm{P}=0.22$ \\
\hline yes & & & & \\
\hline no & 1.0 & & 1.0 & \\
\hline
\end{tabular}

Figure 1. Location of SNs after intratumoural radiotracer injection on the left and right side

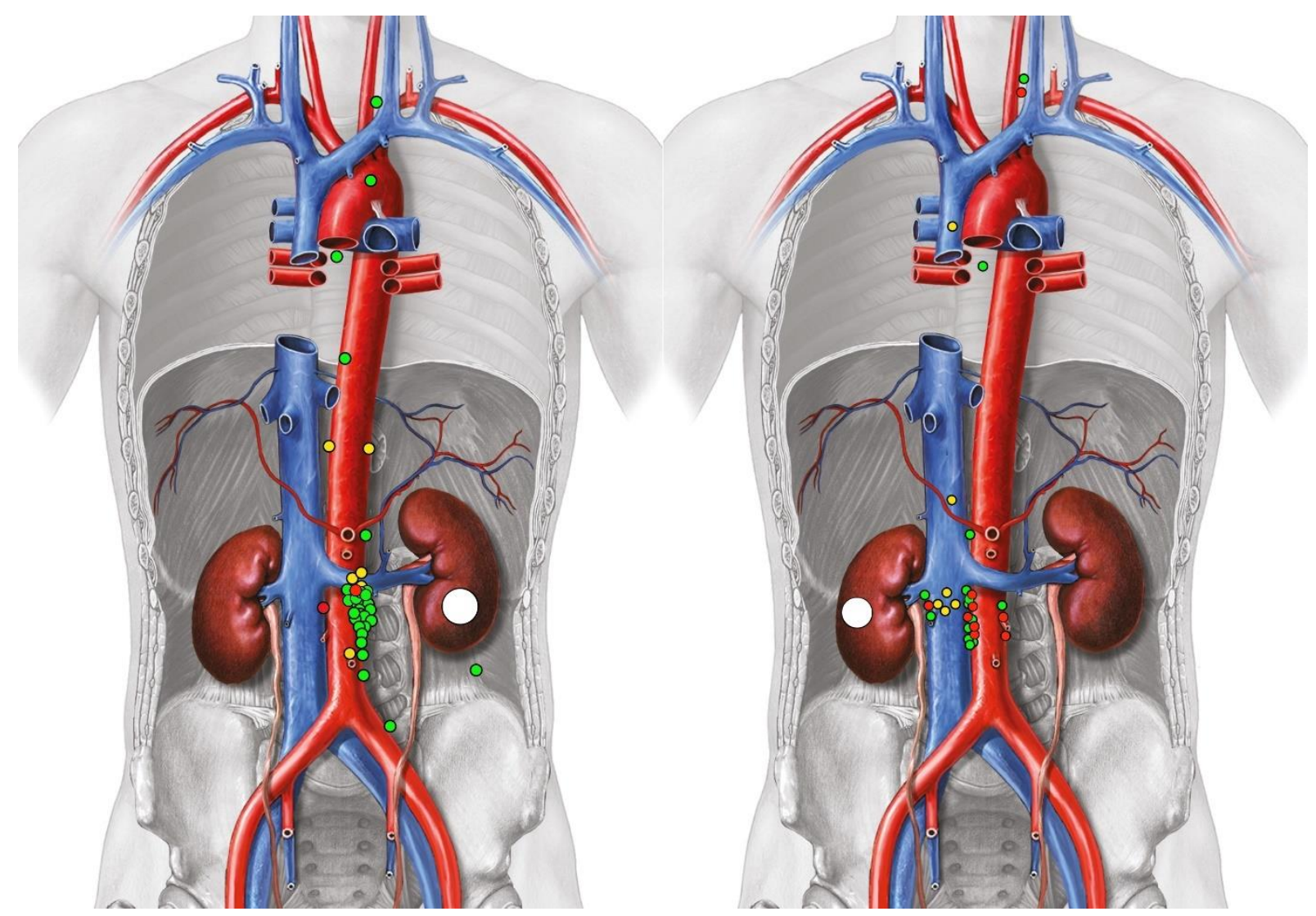

Legend: White dot represents tumour side. Location of SNs is shown with green, yellow and red dots. Green and yellow dots are SNs of patients who remained free of disease (yellow = dorsal from vascular structures). Red dots indicate the location of SNs of patients who developed distant metastases ( $80 \%$ intrathoracic). Image printed with permission of ADAM images. 
Figure 2. Kaplan-Meier curves of metastases free survival of patients with and without interaortocaval SN

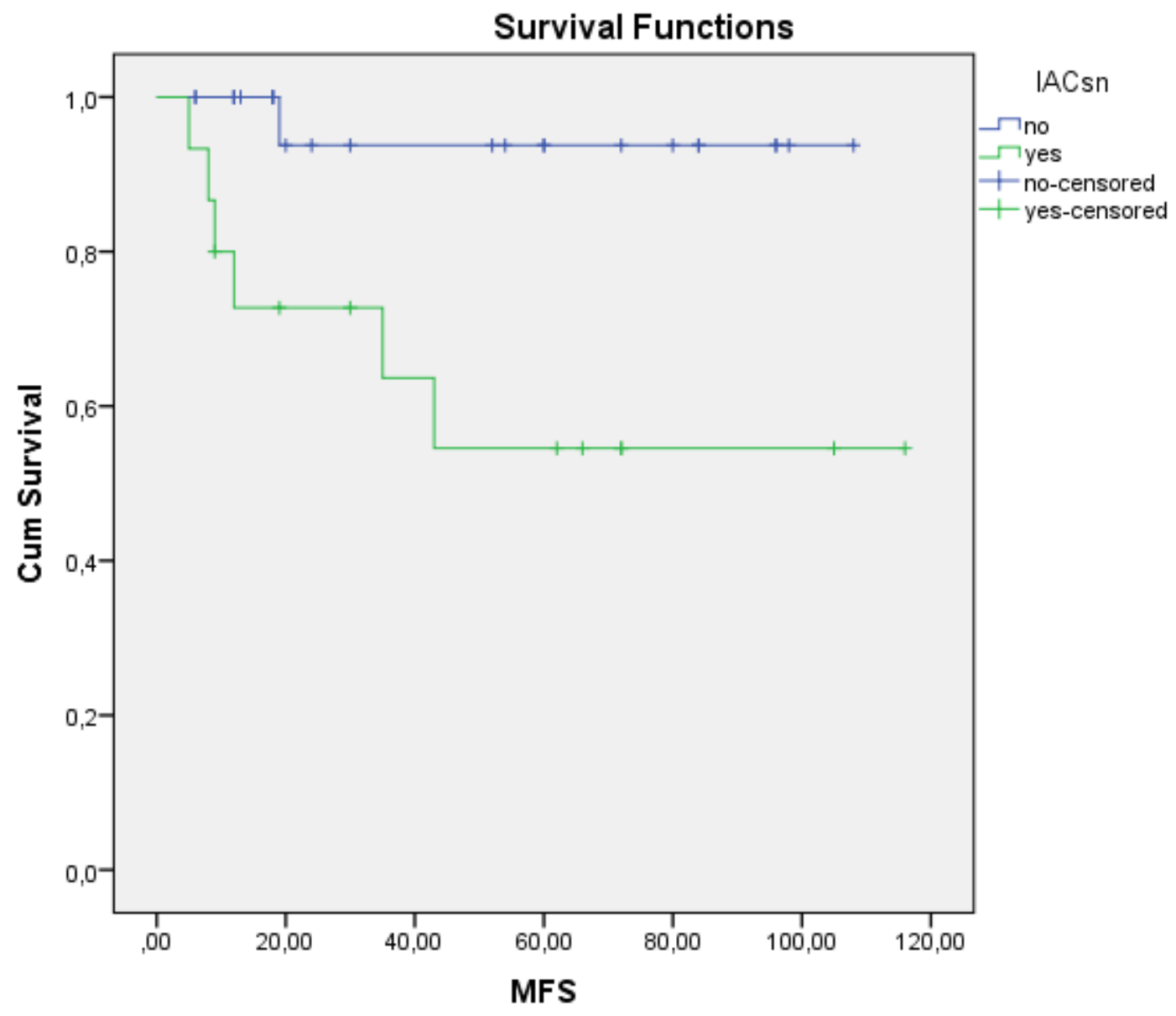

Risk table

\begin{tabular}{|l|l|l|l|l|l|l|}
\hline $\begin{array}{l}\text { Time } \\
\text { (months) }\end{array}$ & 0 & 12 & 24 & 36 & 48 & 60 \\
\hline no & 25 & 19 & 12 & 11 & 11 & 7 \\
\hline yes & 14 & 9 & 8 & 6 & 5 & 5 \\
\hline
\end{tabular}


Figure 3. Lymphatic drainage in renal tumours

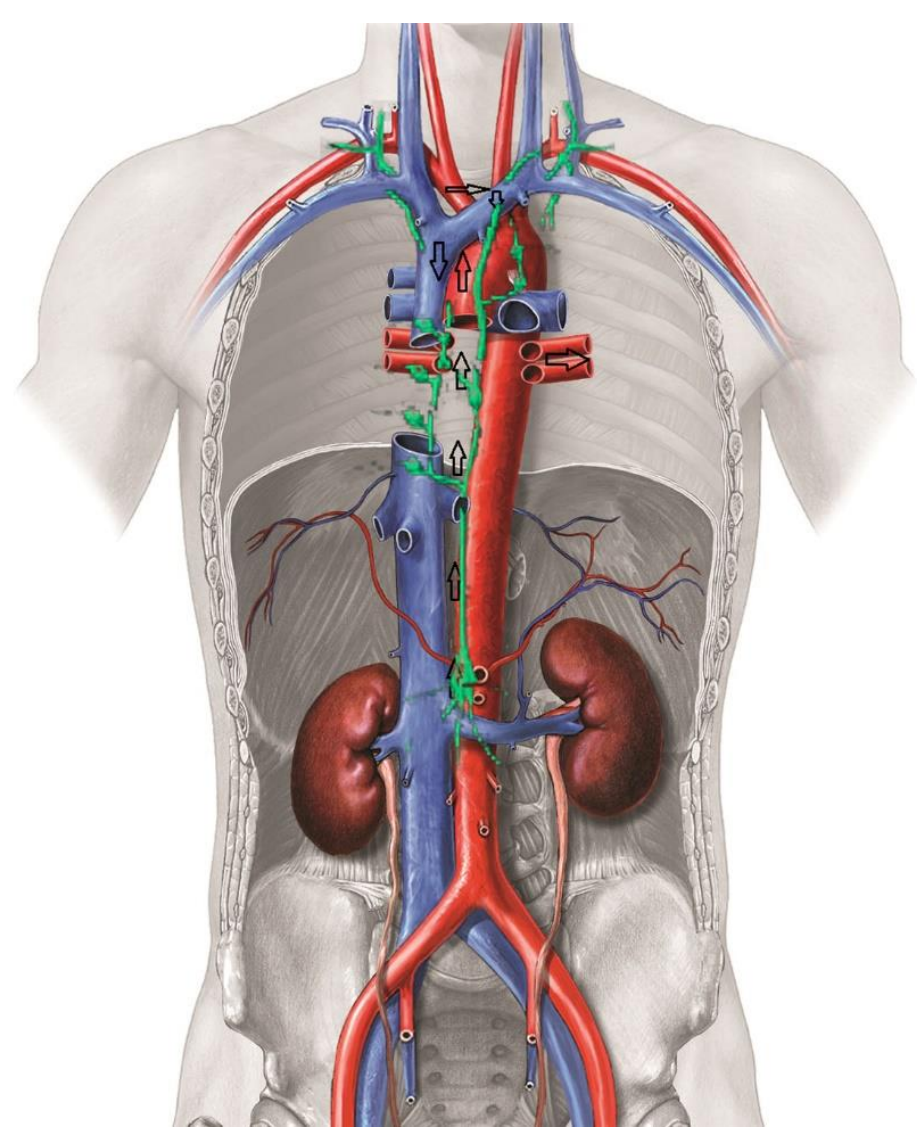

Legend: From retroperitoneal lymph nodes lymph drains directly or indirectly into the thoracic duct where the pressure is higher than in tributaries. Valves prevent lymph flow from the thoracic duct to tributaries and into the thoracic cavity. The thoracic duct terminates into the jugular and subclavian vein and from there circulation of blood takes lymph into the lungs. From the lungs lymph drains into the hilar and mediastinal lymph nodes. Only when the valves in the thoracic duct are insufficient, the lymph may drain directly from the thoracic duct into the mediastinal lymph nodes. The image is printed with permission of ADAM images. 


\section{CHAPTER 4}

Long-term Survival After Resection of Sentinel Node Metastatic Renal Cell Carcinoma.

Kuusk T, De Jong J, Grivas N, Horenblas S, Bex A

Urology. 2017 May;103:e5-e6. 


\section{Summary}

We present a case of a patient who took part in a prospective sentinel lymph node (SN) study to investigate the drainage pattern from renal tumours. The patient was treated with laparoscopic radical nephrectomy and SN lymph node dissection (LND) for a clinically node negative (cNO) left renal tumor of $6 \mathrm{~cm}$ (cT1b). Histopathological examination revealed a papillary type $2 \mathrm{pT} 1 \mathrm{~b}$ renal cell carcinoma (RCC) with two para-aortic metastatic SNs (pN1). The patient is free of disease at 63 months after surgery.

\section{Introduction}

Papillary renal cell carcinoma (pRCC) accounts for 6 to $15 \%$ of all RCC histologic subtypes and is subdivided into type 1 and type 2. Prognosis of type 2 pRCC patients is poor [1, 2], particularly in case of lymph node metastases [3]. Currently, guidelines do not recommend LND as a routine procedure in cNO localized RCC of all subtypes [4]. This recommendation is largely based on a single prospective randomized trial that did not demonstrate a survival benefit for patients undergoing LND versus no LND [5]. However, as the number of patients with lymph node metastases was only $4 \%$, the evidence is rather poor. Other potential reasons for the failure of retroperitoneal LND to demonstrate a survival benefit in RCC may be early haematogenic spread, lymphatic drainage outside the proposed LND templates and direct aberrant drainage through the thoracic duct [6].

To investigate the location of first lymphatic landing sites and their frequency at extraretroperitoneal sites, we performed a phase II SN imaging study enrolling patients with clinically node negative (cNO) renal tumours. The study is approved by the local ethical committee and registered at www.ccmo.nl (NL26406.031.08). We report a case of a patient who took part in the study with preoperatively cNO-disease and was diagnosed with pT1b papillary type $2 \mathrm{RCC}$ and metastatic SN (pN1) after laparoscopic radical nephrectomy combined with SN and non-SN dissection. This case illustrates exceptional long-term survival in conjunction with removal of early occult microscopic lymph node metastases and experimental imaging modalities.

\section{Case presentation}

A 37 year-old male patient underwent whole body computed tomography (CT) for macroscopic hematuria in November 2010. CT imaging revealed a $6 \mathrm{~cm}$ left renal centrally located tumour (cT1b) without metastases (cNOMO). He had no family history of RCC. The laboratory investigations were normal. After informed consent to take part in the prospective SN study, he underwent laparoscopic radical nephrectomy with SN mapping and LND of the left pre-and para-aortal template in January 2011. Preoperatively, ${ }^{99 \mathrm{~m}} \mathrm{Tc}$-nanocolloid was injected percutaneously into the tumour as reported previously [7]. Early preoperative planar lymphoscintigraphy and a fusion with SPECT/CT was performed two hours later revealing para-aortic and simultaneous mediastinal lymphatic drainage (Figure 1a). Matching the SPECT/CT location a cluster of 13 para-aortic SNs was intraoperatively detected at the level of the renal artery by gamma ray detection probe and was excised together with a pre- and para-aortal non-SNs. The mediastinal node was not removed according to protocol. Postoperative recovery was uneventful. 
Histopathological examination revealed a pT1b papillary type 2 RCC (Figure $1 \mathrm{~b}$ ) which was positive for CD10, CK7, RCC, vimentine, AMACR, G250 and negative for CK20, CD117, EMA, MART-1, S100, HMB45. The 2 para-aortic SNs at the level of the renal artery contained metastases and immunohistochemically were positive for CK7 (Figure 1c,1d). The diameter of the largest metastasis was $0.3 \mathrm{~mm}$. A total of 4 other non-SNs in the pre- and distal para-aortic region were benign. DNA gene mutations were not detected by Next Generation Sequencing in a standard cancer hotspot panel. No adjuvant treatment was applied. The patient has been followed up by regular CT scans according to a risk-adapted protocol. The last $\mathrm{CT}$ of the chest and abdomen was performed in April 2016 showing neither distant metastases nor locally recurrent disease.

\section{Discussion}

\section{Presented by Dr Axel Bex, MD, PhD}

PRCC is the second most common histological subtype of renal carcinoma accounting for 6 to $15 \%$ of RCCs [1]. Prognosis of pRCC type 2 is worse than for type 1 [2,8]. Compared to clear cell RCC 5-year cancer specific survival (CSS) after surgical treatment is significantly lower in papillary type 2 RCC (63\% vs $72.4 \%$ ) [9] or even less (50\%) [10]. Data on survival from a recent study of patients treated with sunitinib for metastatic papillary type 1 and 2 RCC demonstrated a median overall survival (OS) of only 12.4 months for type 2 despite systemic therapy [11]. Margulis et al. [12] found that, in case of lymph node metastases after surgically treated pRCC the 5-year CSS is $65 \%$. Interestingly, in this study the course of the disease was relatively indolent. Reasons may be the low number of patients with lymph node metastatic pRCC and that half of the cases were papillary type 1 . In addition, patients in this study underwent LND. Conversely, studies revealed poor survival even for localized disease. Pignot et al. [13] reported a 5-year overall and disease-free survival rate for surgically treated patients without lymph node involvement of $55 \%$ and $44 \%$ in pRCC type 2 tumors. Steffens et al. [3] has reported papillary subtype as a significant positive prognostic factor in localized but a negative prognostic factor in metastatic $(\mathrm{N}+$ or/and $\mathrm{M}+)$ tumour stages at the time of surgery. In this study pRCC is not subtyped but for all pRCC 5-year OS rate was only $15.9 \%$ in case of metastatic disease.

Our patient revealed occult para-aortic lymph node metastases after excision of a clinically lymph node negative localized renal tumor in the frame of a prospective study investigating lymphatic drainage of renal tumours. According to current guidelines lymphadenectomy would not have been recommended [4]. Interestingly, the small metastases (maximum 0,3 $\mathrm{mm}$ ) were found in two retroperitoneal SNs, which are by definition the lymph nodes that received direct drainage from the tumour. The reason why the mediastinal SN was not removed was because the primary endpoint of the study is drainage on imaging. Since the procedure of $\mathrm{SN}$ dissection remains experimental and to protect patients from undue surgical morbidity, only nodes accessible through the nephrectomy approach are to be sampled according to our protocol. The long-term recurrence-free survival of 5years despite a poor-prognostic histological subtype suggests that individual patients with early and therefore small lymph node metastases could benefit from lymphadenectomy.

The frequency of occult potentially curable lymph node metastases in clinically node negative patients is unknown. However, the $4 \%$ lymph node metastases rate in the EORTC study which compared LND to no LND in patients with CT1-T3 CNO MO RCC of all subtypes suggests that it is a rare event [5]. A recent study showed that flow cytometry was able to detect micro metastatic RCC in lymph nodes that were undetected by conventional hematoxylin-eosin microscopy suggesting that low-volume lymph node metastases are more frequent than previously thought [14]. We know little 
about differences in metastatic landing sites with regard to the various histologic subtypes of RCC. A study investigated the distribution of metastases in pRCC. Ronnen et al. [15] evaluated the metastatic sites in 38 patients with metastatic pRCC and observed that lymph nodes were the most frequent site (retroperitoneum $61 \%$; mediastinum $47 \%$ ), followed by lung $(47 \%)$ and bone (32\%). An autopsy study of 1828 cases including various subtypes observed the highest rate of lymph node metastases at the pulmonary hilum (66\%) followed by retroperitoneal (36\%), para-aortal $(26 \%)$ and supraclavicular (20\%) lymph nodes [16]. Lack of data and contradictory findings cause uncertainty and challenges regarding potential dissimilarities in metastatic sites of different subtypes of RCC. Some subtypes of RCC may benefit more from locoregional LND than others even in clinically lymph node negative disease. Investigating the pattern of lymph node drainage in different subtypes of RCC and an association of a potential survival benefit with removing lymphatic landing sites may lead to new concept in the surgical treatment of RCC, but the clinical role of sentinel node identification and resection in this disease is currently unknown.

In conclusion, this case suggests that patients with clinically node-negative RCC may benefit from removal of locally occult lymph node metastases and that sentinel node imaging may help to identify these nodes. Uncertainties and knowledge gaps regarding frequency of occult lymph node metastases and the outcome of their removal in various subtypes of RCC warrant further investigation of the $\mathrm{SN}$ concept.

Acknowledgements: We would like to thank the European Urological Scholarship Programme for financially supporting the research fellowship of Teele Kuusk at Antoni van Leeuwenhoek Hospital/ the Netherlands Cancer Institute in Amsterdam, the Netherlands.

Conflict of interest: No conflicts of interest.

\section{References:}

1. Keegan KA, Schupp CW, Chamie K, Hellenthal NJ, Evans CP, Koppie TM. Histopathology of surgically treated renal cell carcinoma: survival differences by subtype and stage. J Urol. 2012;188: 391.

2. Lee JH, Choi JW, Kim YS. The value of histologic subtyping on outcomes of clear cell and papillary renal cell carcinomas: a meta-analysis. Urology. 2010;76(4):889-94.

3. Steffens $\mathrm{S}$, Janssen $\mathrm{M}$, Roos FC, et al. Incidence and long-term prognosis of papillary compared to clear cell renal cell carcinoma-a multicentre study. Eur J Cancer. 2012;48(15):2347-52.

4. Ljungberg B, Bensalah K, Canfield S, et al. EAU guidelines on RCC-an update 2014. Eur Urol. 2015;67(5):913-24.

5. Blom JHM, van Poppel H, Maréchal JM, et al. Radical nephrectomy with and without lymph-node dissection: final results of European Organization for Research and Treatment of Cancer (EORTC) randomized phase 3 trial 30881. Eur Urol. 2009;55:28-34.

6. Brouwer OR, Noe A, Olmos RA, Bex A. Lymphatic drainage from renal cell carcinoma along the thoracic duct visualized with SPECT/CT. Lymphat Res Biol. 2013;11:233-8. 
7. Bex A, Vermeeren L, de Windt G, Prevoo W, Horenblas S, Olmos RA. Feasibility of sentinel node detection in renal cell carcinoma: a pilot study. Eur J Nucl Med Mol Imaging. 2010; 37: 1117-23.

8. Leibovich $\mathrm{BC}$, Lohse $\mathrm{M}$, Crispen $\mathrm{PL}$, et al. Histological subtype is an independent predictor of outcome for patients with renal cell carcinoma. J Urol. 2010; 183: 1309.

9. Simone G, Tuderti G, Ferriero M, et al. Papillary type 2 versus clear cell renal cell carcinoma: Survival outcomes. Eur J Surg Oncol. 2016; doi: 10.1016/j.ejso.2016.08.003.

10. Yamashita S, Ioritani N, Oikawa K, Aizawa M, Endoh M, Arai Y. Morphological subtyping of papillary renal cell carcinoma: clinicopathological characteristics and prognosis. Int J Urol. 2007;14: 679-83.

11. Ravaud A, Oudard S, De Fromont M, et al. First-line treatment with sunitinib for type 1 and type 2 locally advanced or metastatic papillary renal cell carcinoma: a phase II study (SUPAP) by the French Genitourinary Group (GETUG). Ann Oncol. 2015;26(6):1123-8.

12. Margulis V, Tamboli P, Matin SF, Swanson DA, Wood CG. Analysis of clinicopathologic predictors of oncologic outcome provides insight into the natural history of surgically managed papillary renal cell carcinoma. Cancer. 2008;112(7):1480-8.

13. Pignot G, Elie C, Conquy $\mathrm{S}$, et al. Survival analysis of 130 patients with papillary renal cell carcinoma: prognostic utility of type 1 and type 2 subclassification. Urology. 2007; 69 (2):230-5.

14. Hartana CA, Kinn J, Rosenblatt R, et al. Detection of micrometastases by flow cytometry in sentinel lymph nodes from patients with renal tumours. Br J Cancer. 2016; doi:

10.1038/bjc.2016.279.

15. Ronnen EA, Kondagunta GV, Ishill N, et al. Treatment outcome for metastatic papillary renal cell carcinoma patients. Cancer. 2006; 107:2617-21.

16. Saitoh H, Nakayama M, Nakamura K, Satoh T. Distant metastasis of renal adenocarcinoma in nephrectomized cases. J Urol. 1982;127:1092-5. 


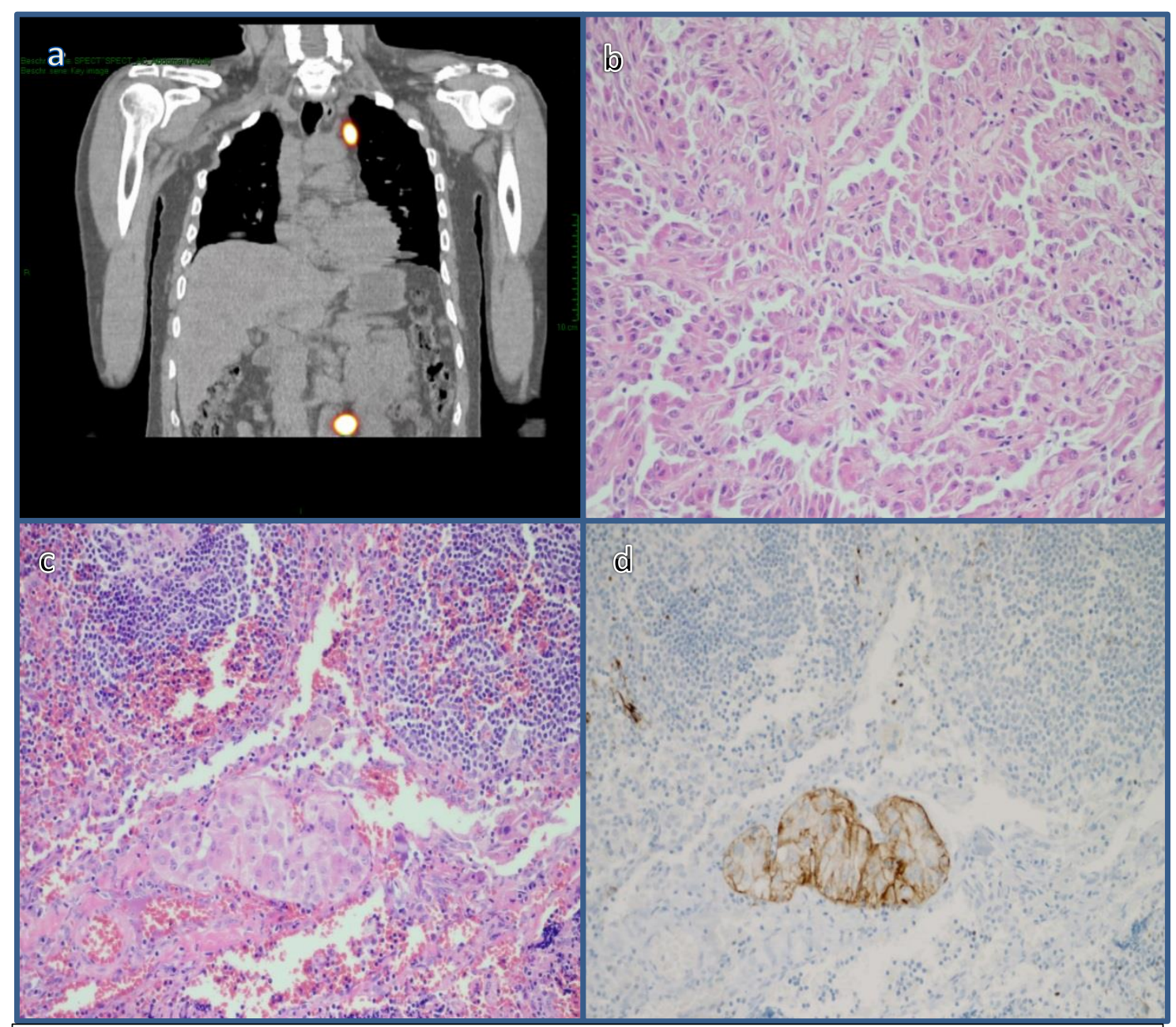

Figure $1 \mathrm{~A}$. Para-aortal and mediastinal sentinel lymph nodes on SPECT-CT imaging; B. Primary type II pRCC with eosinophilic cytoplasm, large nuclei and prominent nucleoli $(H \& E, \times 200) ; C$. Type II pRCC metastases in a lymph node $(H \& E, \times 200)$; D. Immunohistochemically positive sentinel lymph node for CK7 (x200). 


\section{CHAPTER 5}

Topographic distribution of first landing sites of lymphatic metastases from patients with renal cancer

Kuusk Teele, Zondervan Patricia, Lagerveld Brunolf, Rosenzweig Barak, Raman Avi, Blok Joost M, Roderick de Bruijn, Niels Graafland, Kees Hendricksen, Umberto Capitanio, Andrea Minervini, Borje Ljungberg, Simon Horenblas, Bex Axel

Urol Oncol. 2020 May;38(5):521-525. 


\section{Abstract}

Introduction \& Objectives

Adjuvant studies with checkpoint inhibitors have attracted new interest in accurate pathological lymph node (LN) staging in renal cell carcinoma (RCC). Sentinel lymph node (SN) studies in cNO patients revealed the pattern of lymphatic radiotracer drainage from renal tumours. The aim of this study was to describe the location of single- or oligo-metastatic $L N$ and analyze if the topography of these first landing sites matches the drainage pattern observed in SN studies of renal tumours.

Materials \& Methods

We collected data from 8 referral centers from 1990-2018 of all patients with pT1-4 cN0 or cN1 M0 RCC with pathologically confirmed single- or oligo-metastases in locoregional LN. The location of LN metastases, number, size of metastatic $L N$ and survival were analyzed using descriptive statistics with SPSS version 22 (IBM, Chicago, IL, USA).

\section{Results}

From 3794 patients with histologically confirmed pN1, a total of 76 patients (2\%) with single- or oligometastatic pN1 were identified, of whom $24(31.6 \%)$ and 52 (68.4\%) were cNO and cN1, respectively. On the left side, LN metastases were predominantly located in the paraaortal (48.0\%; 95\% $\mathrm{Cl} 29.22-63.12 \%)$ and hilar $(31.42 \%$; $95 \% \mathrm{Cl} 17.4-49.4 \%)$ area. On the right side, metastases located in retrocaval $(26.82 \%(95 \% \mathrm{Cl} 14.7-43.2 \%))$, hilar $(26.82 \%(95 \% \mathrm{Cl} 14.7-43.2 \%))$, interaortocaval $(26.82 \%(95 \% \mathrm{Cl} 14.7-43.2 \%))$ and paracaval $(17.07 \%(95 \% \mathrm{Cl} 7.6-32.6 \%))$ area. These landing sites exactly matched the lymphatic drainage pattern of intratumorally injected radiotracer reported in SN studies for both sides.

\section{Conclusions}

Single- or oligo-metastatic LNs in renal cancer are mainly located in the hilar, retro-, para and interaortocaval region on the right side and paraaortal region on the left side. These first landing sites match the drainage pattern reported in SN trials. 


\section{Introduction}

Lymph node dissection (LND) has not been shown to improve survival in renal cancer in a randomized controlled trial and large retrospective studies [1,2]. However, LND is regaining importance with novel checkpoint inhibitor and combination trials where staging and detection of micrometastatic disease is of interest. Yet, LND templates are poorly defined and recent lymphatic drainage studies after injection of radiotracer into renal tumours showed that lymphatic drainage does also occur outside previously suggested LND templates [3]. For these reasons it is of importance to accurately define the LND template for renal carcinoma or find other applicable means for LN staging. One technique adopted in many cancer types for $L N$ staging is a sentinel lymph node biopsy (SLNB), which provides an opportunity to investigate the resected sentinel LNs in micrometer sections in search of occult metastases [4]. Even though the clinical significance of occult LN metastases is not well defined, the information gained from SN imaging for locating or mapping the first landing sites may be of relevance for LND performed for staging purposes for adjuvant trials [4]. The rationale for $\mathrm{LN}$ staging in adjuvant trial settings is to accurately assess high-risk disease because even isolated LN metastases bear an extremely poor prognosis $[5,6,7,8]$.

For the purpose of accurate $\mathrm{pN}$-staging for adjuvant trials, standardization of a LND template for renal cancer based on the location of occult $L N$ metastases would be required. A prospective lymphatic drainage study was performed earlier but only very few patients harbored occult metastases in the sentinel nodes (SN) [3]. Likewise, publications on the pattern of single site LN metastases in renal cancer are rare.

Therefore, the aim of this study was to describe the location of single- or oligo-metastatic LNs and analyze if the topography of these first landing sites matches the drainage pattern observed after intratumoural radiotracer injection in SN studies of renal tumours [3].

\section{Materials \& Methods}

After institutional review board approval, we collected data from 8 different centers from 1990-2018 of all patients with pT1-4 MO RCC with pathologically confirmed single- or oligo-metastases in locoregional LNs, presenting as either occult LN metastasis (cNO) or a single $\mathrm{CN} 1$ of $>1$ and $<2 \mathrm{~cm}$ node on imaging. For the purpose of this study oligometastatic locoregional LN were defined as $</=3$ LN metastases at pathological staging (pN1) in the same cluster. Participating centers performed LND for $\mathrm{CNO}$ and $\mathrm{CN} 1$ on the right side from the renal hilum and artery, including the hilar paracaval and retrocaval nodes, down to the common iliac artery, including the interaortocaval nodes and on the left side from the renal artery including left paraaortal nodes either down to the common iliac artery or the inferior mesenteric artery, which was also the boundary for those centers including preaortal and interaortocaval lymph nodes in their left sided templates (supplementary table 1). Any cN1 nodes detected on imaging outside these boundaries were additionally resected. All LNDs were performed as open procedures. Collected data included location of the LN metastases, number, size of metastatic LNs and survival. The main objective of the study was to assess the first landing sites of primary LN metastases. Secondary objective of the study was the overall survival (OS) of a subgroup of patients with early occult LN metastases cNO-pN1.

We analyzed the data using descriptive statistics showing the location of metastatic LNs and KaplanMeier survival models for evaluating survival differences between cNO/pN1 and cN1/pN1 cases, using SPSS version 22 (IBM, Chicago, IL, USA). 


\section{Results}

From 3794 patients with histologically confirmed pN1 disease, we identified a total of 76 patients (2\%), with single- or oligometastatic LN, of whom 24 (31.6\%) and 52 (68.4\%) were cNO and cN1 at diagnosis, respectively (table 1 ). The majority $(60 ; 78.9 \%$ ) had clear cell RCC. The right side was involved in 41 (53.9\%) and the left side in 35 (46.1\%) patients. The median number of detected LN metastases at LND was 2.0 (Interquartile range [IQR] 1-3) with a median number of LNs removed of 4.0 (IQR 2-7). On the left side, LN metastases were predominantly located in the paraaortal (48.0\%; (95\% Cl 29.22-63.12\%)) and hilar (31.42\%; (95\% Cl 17.4-49.4\%)) area, matching the paraaortal and hilar radiotracer drainage observed in SN studies (figure and table 1$)$. In 6 patients $(17.1 \%(95 \% \mathrm{Cl}$ 6.6-33.6\%) LN metastasis crossed over to the right paraaortal side. This interaortocaval location was not associated with number of LN removed. On the right side, metastases located in retrocaval (26.82\% (95\% Cl 14.7-43.2\%)), hilar (26.82\% (95\% Cl 14.7-43.2\%)), interaortocaval (26.82\% (95\%Cl 14.7-43.2\%)) and paracaval $(17.07 \%(95 \% \mathrm{Cl} 7.6-32.6 \%))$ area, following the drainage pattern reported in SN studies on the right side (figure and table 2). One patient had a LN metastasis which crossed over to the left paraortic side which had been $\mathrm{cN} 1$ on imaging. The median OS for $\mathrm{CNO}$ was 58.0 months (HR 2.34 95\% Cl 53.40-62.59) and 32.0 months (HR $5.5595 \% \mathrm{Cl}$ 21.11-42.88) with $\mathrm{p}=0.12$ for $\mathrm{CN} 1$, respectively (figure 3 ).

\section{Discussion}

Following a disease-free survival advantage with sunitinib over placebo as an adjuvant treatment after nephrectomy for high risk renal cancer patients, the Food and Drug Administration (FDA) approved the drug for this indication in 2017, whereas the European Medicines Agency (EMA) did not [9]. In the study, high risk was defined by T stage, grade, performance status and LN status, provided LND was performed [9]. Recently, it has been suggested that knowledge of pathological LN status is required for more accurate staging and prediction of progression after surgery [10]. LN positive cases had a two-fold increased likelihood of progression and overall mortality compared to LN negative cases [10]. Therefore, adjuvant trials have attracted a new interest in LND for staging purposes in patients with clinically negative nodes ( $\mathrm{CNO}$ ) on imaging. However, a standardized and validated LND template for renal cancer does not yet exist. Ideally, LND templates for patients with cNO RCC cover areas including the LNs receiving direct lymphatic drainage from the tumour. A lymphatic drainage mapping study revealed recently that $65 \%$ of the first $L N$ s receiving intratumorally injected radiotracer are locoregionally inside the suggested historical templates $[3,11]$. However, in this SN study only very few patients had occult LN metastases in the SNs receiving the radiotracer. It is therefore unproven if these radiotracer-active SN would generally be identical with the first metastatic landing sites.

Unfortunately, most publications on the location of $L N$ metastases in the retroperitoneum are inappropriate to define first landing sites because they reported on patients with multiple LN metastases. Given the extensive lymphatic connections in the retroperitoneal space it is impossible to reiterate which of the multiple LN metastases was the first to receive direct lymphatic drainage from the tumour. We therefore hypothesized that isolated single- or oligometastatic LNs in patients with $\mathrm{cNO}$ or very limited $\mathrm{cN} 1$ staging on imaging represent the first landing sites and investigated in this retrospective cohort if their topography matches the location of the SNs in the radiotracer studies.

Although large series of patients with LN metastases have been published, isolated LN metastases especially in CNO RCC are rare. It required cooperation with 8 centres to collect sufficient cases for this analysis. Nevertheless, we demonstrated consistency in the pattern of isolated LN metastases 
with the location of SNs in the radiotracer mapping study [3]. We showed that locoregionally on the right side the majority of the single LN metastases were located in hilar, para-retro- and interaortocaval nodes and on the left side predominantly in hilar and paraaortic nodes, although isolated LN metastases were found on the right side of the aorta in the interaortocaval space in up to $17 \%$.

Only one other retrospective study described the location of isolated LN metastases from RCC [12]. Contrary to our analysis in patients with isolated synchronous LN metastases at the time of nephrectomy, the 35 patients had histologically proven metachronous isolated LN metastatic disease. Although this study has shown a similar location of LN metastases, some of the metastatic sites were outside the commonly used LND templates. Predominant locations were paraaortal and interaortocaval nodes on the left, and interaortocaval and paracaval nodes on the right side [12]. However, retroaortal LN metastases on the right and a substantial number of interaortoacaval LN metastases on the left side are worth noticing [12]. Another study showed that $29 \%$ of enlarged LNs on imaging are located in areas which are away from the primary landing sites and are therefore "skipped" LNs [13]. In our study, we also found LN metastases in areas beyond commonly recommended templates including para- and preaortal LNs on the right and interaortocaval LNs on the left side. These LNs are not always considered part of the LND templates in renal cancer [11] and this pattern confirms the unpredictability of lymphatic drainage in renal tumours. A LND template that covers all potential first lymphonodular metastatic landing sites would induce further surgical complexity which encourages using SN technology and SPECT/CT.

In addition to the absence of a survival benefit for LND in a randomized trial, available data regarding removal of isolated $L N$ metastases in renal cancer are retrospective and contradictory [2, $12,14,15,16,17]$. Some studies suggest a potential survival advantage in patients with cN0 tumour stages who had a single occult $L N$ removed $[5,12]$. However, in one of these studies only $30 \%$ were $\mathrm{MO}$ and the median size of the LN metastases was $2.6 \mathrm{~cm}$ (IQR 1.9-5), which does not support that these were early landing sites [12]. In our cohort, we compared the OS of cNO and cN1 patients because we hypothesized that patients with occult LN metastases (cNO-pN1) may have a better survival after LND of these isolated metastases. Interestingly, there was a trend towards longer OS in those isolated cases when compared to the cN1-pN1 patients but which was not significantly different. However, the number of patients and events were too small for drawing any ultimate conclusions. In addition, with only $2 \%$ of all patients with pN1 it is evident that isolated single- or oligometastatic LN in RCC is a rare event.

The study has several limitations. There is no universally accepted template for LND in renal cancer. The data is subject to retrospective bias as we did not prospectively control for a uniform and complete template-based LND. Nevertheless, these patients were specially selected because they had very early lymphonodular involvement either testified by a single cN1 lymph node on imaging < 2 $\mathrm{cm}$ or $\mathrm{CNO}$ pN1 disease in no more than 3 adjacent nodes. We cooperated with the centers which, contrary to EAU guideline recommendations, chose to perform LND in various templates for intermediate/high-risk cNO and cN1 renal cancer. In this retrospective, multicenter study, LND templates varied with loco regional dissection templates incorporating on the left side the hilar and paraaortic lymph nodes from the renal artery to the inferior mesenteric in 1 center and to the common iliac in 7 centers with additional inclusion of the preaortal and interaortocaval nodes down to the inferior mesenteric. However, on the right side the resection boundaries were more uniform including for all centers the hilar nodes along the renal artery (paracaval and retrocaval) and interaortocaval lymph nodes down to the common iliac. Owing to the retrospective nature of the analysis pathologists would not investigate lymph node status according to a universal protocol 
which may explain the low median number of LN removed at LND. This reduces the certainty that not more nodes were involved. However, most centers offered LND specimen to pathology in packages separated by anatomical regions (supplementary table 1 ).

\section{Conclusions}

Early single- or oligo-metastatic LNs in renal cancer are mainly located in the hilar, retro-para and interaortocaval region on the right side and the paraaortal region on the left side. These locations match with the drainage pattern reported in $\mathrm{SN}$ trials. If current adjuvant trials with immune checkpoint inhibition demonstrate a survival benefit over placebo, LND within these templates may regain an indication for proper risk- and prognosis assessment and techniques to identify first landing sites of occult LN metastases may reduce the need for extensive templates.

\section{References:}

1. Blom JH, van Poppel H, Maréchal JM, Jacqmin D, Schröder FH, de Prijck L, et al. Radical nephrectomy with and without lymph-node dissection: final results of European Organization for Research and Treatment of Cancer (EORTC) randomized phase 3 trial 30881.Eur Urol. 2009;55(1):2834.

2. Bhindi B, Wallis CJD, Boorjian SA, Thompson RH, Farrell A, Kim SP, et al. The role of lymph node dissection in the management of renal cell carcinoma: a systematic review and meta-analysis. BJU Int. 2018;121(5):684-698.

3. Kuusk T, De Bruijn R, Brouwer RO, De Jong J, Donswijk M, Grivas N, et al (2018) Lymphatic drainage from renal tumors in vivo: a prospective sentinel node study using SPECT/CT imaging. J Urol 199(6):1426-1432

4. Mamounas EP, Kuehn T, Rutgers EJT, Von Minckwitz G. Current approach of the axilla in patients with early-stage breast cancer. The Lancet 2017 DOI: 10.1016/S0140-6736(17)31451-4

5. Gershman B, Moreira DM, Thompson RH, Boorjian SA, Lohse CM, Costello BA, Cheville JC, Leibovich BC. Renal Cell Carcinoma with Isolated Lymph Node Involvement: Long-term Natural History and Predictors of Oncologic Outcomes Following Surgical Resection. Eur Urol.

2017;72(2):300-306.

6. Babaian KN, Kim DY, Kenney PA, Wood CG Jr, Wong J, Sanchez C, et al. Preoperative predictors of pathological lymph node metastasis in patients with renal cell carcinoma undergoing retroperitoneal lymph node dissection. J Urol. 2015;193(4):1101-7.

7. Tilki D, Chandrasekar T, Capitanio U, Ciancio G, Daneshmand S, Gontero P, et al. Impact of lymph node dissection at the time of radical nephrectomy with tumor thrombectomy on oncological outcomes: Results from the International Renal Cell Carcinoma-Venous Thrombus Consortium (IRCCVTC). Urol Oncol. 2018;36(2):79.e11-79.e17.

8. Gershman B, Thompson RH, Boorjian SA, Larcher A, Capitanio U, Montorsi F, et al. Radical Nephrectomy with or without Lymph Node Dissection for High Risk Nonmetastatic Renal Cell Carcinoma: A Multi-Institutional Analysis.J Urol. 2018;199(5):1143-1148. 
9. Ravaud A, Motzer RJ, Pandha HS, et al: Adjuvant sunitinib in high-risk renal-cell carcinoma after nephrectomy. N Engl J Med 2016; 375: pp. 2246-2254

10. Capogrosso P, Larcher A, Nini A, Muttin F, Cianflone F, Ripa F, Briganti A, et al. The critical role of lymph node dissection in selecting high-risk nonmetastatic renal cancer candidates for adjuvant therapy after nephrectomy.Urol Oncol. 2019 doi: 10.1016/j.urolonc.2019.01.009.

11. Karmali RJ, Suami H, Wood CG, Karam JA. Lymphatic drainage in renal cell carcinoma: back to the basics. BJU Int. 2014 Dec;114(6):806-17.

12. Russell CM, Espiritu PN, Kassouf W, Schwaab T, Buethe DD, Dhilon J, et al. Surgical Outcomes in the Management of Isolated Nodal Recurrences: A Multicenter, International Retrospective Cohort. J Urol. 2014;192(2):350-6.

13. Hadley DA, Stephenson RA, Samlowski WE, Dechet CB. Patterns of enlarged lymph nodes in patients with metastatic renal cell carcinoma. Urol Oncol. 2011;29(6):751-5.

14. Ristau BT, Manola J, Haas NB, et al: Retroperitoneal Lymphadenectomy for high risk, nonmetastatic renal cell carcinoma: an analysis of the ASSURE (ECOG-ACRIN 2805) adjuvant trial. J Urol 2018;199:53-59.

15. Russell CM, Lue K, Fisher J, Kassouf W, Schwaab T, Sexton WJ, et al. Oncological control associated with surgical resection of isolated retroperitoneal lymph node recurrence of renal cell carcinoma. BJU Int. 2016;117(6B):E60-6.

16. Boorjian SA, Crispen PL, Lohse CM, Leibovich BC, Blute ML. Surgical resection of isolated retroperitoneal lymph node recurrence of renal cell carcinoma following nephrectomy. J Urol. 2008;180(1):99-103;

17. Nini A, Larcher A, Cianflone F, Trevisani F, Terrone C, Volpe A, et al. The Effect of Anatomical Location of Lymph Node Metastases on Cancer Specific Survival in Patients with Clear Cell Renal Cell Carcinoma. Front Surg. 2018; 28;5:26. 
cNO-pN1

cN1-pN1

Table 1. Patient and tumor characteristics

\begin{tabular}{|c|c|c|c|}
\hline Number of patients (\%) & 76 & $24(31.6 \%)$ & $52(68.4 \%)$ \\
\hline Median age (IQR) & $62(50-71)$ & $54(44-72)$ & $65(54-71)$ \\
\hline Median tumour size in cm (IQR) & $9(5-12)$ & $9(7.1-12)$ & $9(4.9-12)$ \\
\hline \multicolumn{4}{|l|}{ pT stage (number of patients) } \\
\hline T1a & $7(9.21 \%)$ & 1 & 6 \\
\hline $\mathrm{T} 1 \mathrm{~b}$ & $10(13.15 \%)$ & 4 & 6 \\
\hline $\mathrm{T} 2 \mathrm{a}$ & $6(7.89 \%)$ & 4 & 2 \\
\hline $\mathrm{T} 2 \mathrm{~b}$ & $12(15.78 \%)$ & 6 & 6 \\
\hline T3a & $14(18.42 \%)$ & 3 & 11 \\
\hline $\mathrm{T} 3 \mathrm{~b}$ & $14(18.42 \%)$ & 3 & 11 \\
\hline T4 & $7(9.21 \%)$ & 2 & 5 \\
\hline Right side & $41(53.94 \%)$ & 10 & 31 \\
\hline Left side & $35(46.05 \%)$ & 14 & 21 \\
\hline \multicolumn{4}{|l|}{ Histology (number of patients, \%) } \\
\hline Clear cell RCC & $59(77.63 \%)$ & 18 & 41 \\
\hline \multicolumn{4}{|c|}{ Fuhrman grade (number of patients) } \\
\hline II & 13 & 7 & 6 \\
\hline III & 25 & 8 & 17 \\
\hline IV & 11 & 3 & 8 \\
\hline Missing data & 10 & 4 & 6 \\
\hline Papillary type 1 RCC & $6(7.89 \%)$ & 2 & 4 \\
\hline Papillary type 2 RCC & $4(5.26 \%)$ & 1 & 3 \\
\hline Chromophobe RCC & $3(3.94 \%)$ & 2 & 1 \\
\hline NOS & $3(3.94 \%)$ & 1 & 2 \\
\hline Medullary & $1(1.31 \%)$ & 0 & 1 \\
\hline
\end{tabular}




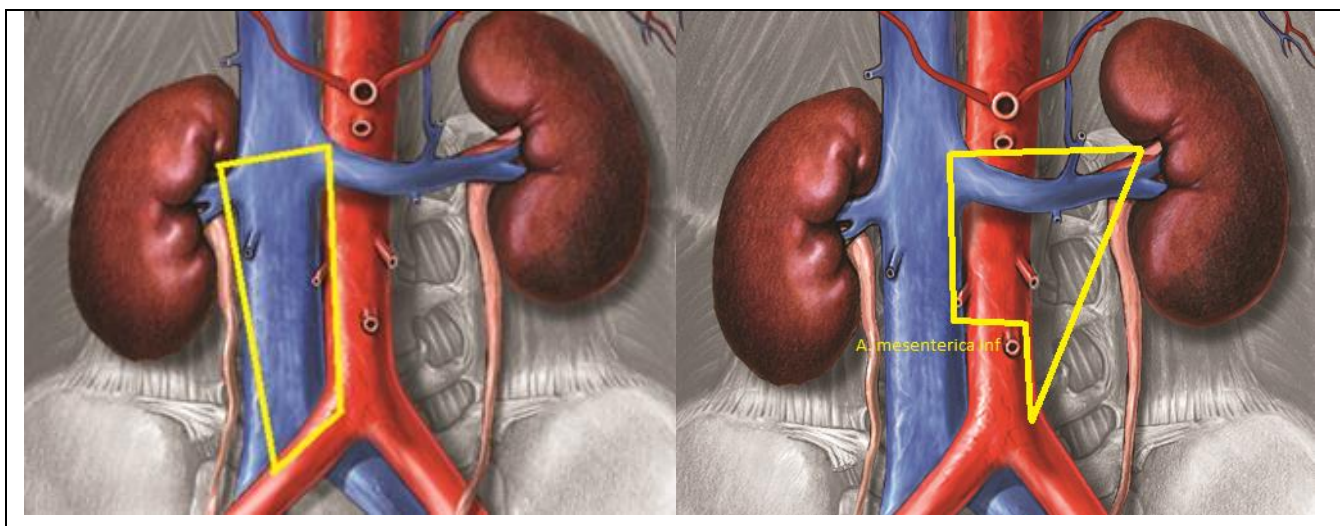

Hospitals using LND templates including on the right side the LN from the hilum, along the renal artery (hilar paracaval and retrocaval) and interaortocaval down to the common iliac artery and on the left side hilar, preaortal and interaortocaval down to the inferior mesenteric and paraaortal down to the common iliac*:

1. San Raffaele Scientific Institutute, Milan, Italy

2. Umeå University Hospital, Umeå, Sweden

3. OLVG, Amsterdam, The Netherlands

4. Careggi Hospital, Florence, Italy

5. Academic Medical Center, Amsterdam, The Netherlands

6. AvL Netherlands Cancer Institute, Amsterdam, The Netherlands

7. John Hunter Hospital, Newcastle, Australia

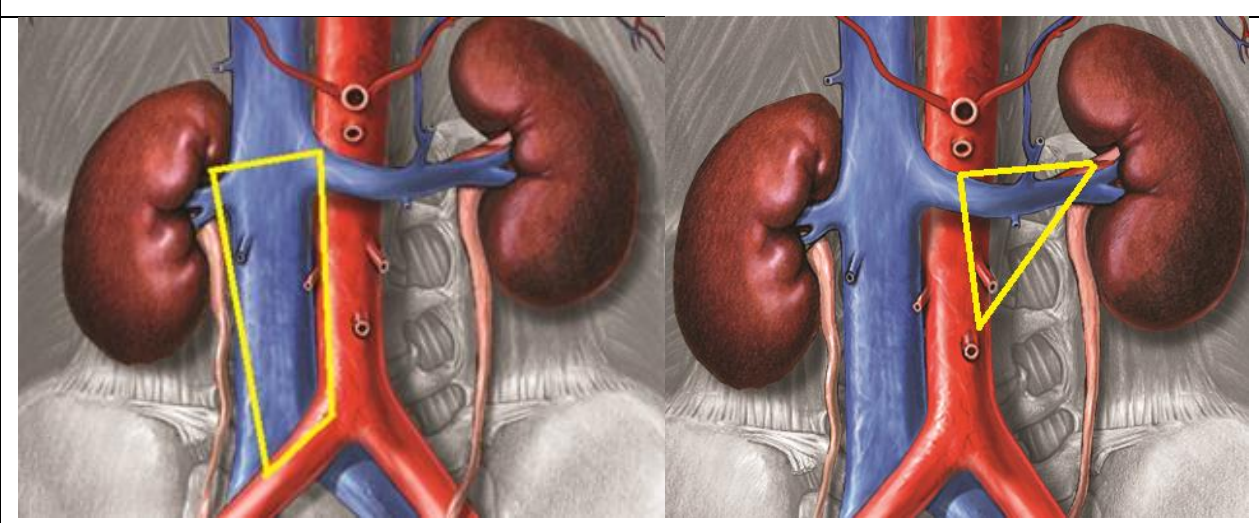

Hospital using LND templates including on the right side the LN from the hilum, along the renal artery, (hilar paracaval and retrocaval) and interaortocaval down to the common iliac artery and on the left side hilar and paraaortal down to the inferior mesenteric artery*:

1. The Chaim Sheba Medical Center, Ramat Gan, Israel 
LN sent en bloc to pathologist

1. Careggi Hospital, Florence, Italy

2. OLVG, Amsterdam, The Netherlands

LN sent to pathologist separated by anatomical regions (on the right:

hilar/retro/paracaval or interaortocaval; on the left: hilar or paraaortal)

1. San Raffaele Scientific Institutute, Milan, Italy

2. Umeå University Hospital, Umeå, Sweden

3. The Chaim Sheba Medical Center, Ramat Gan, Israel

4. OLVG, Amsterdam, The Netherlands

5. Academic Medical Center, Amsterdam, The Netherlands

6. AvL Netherlands Cancer Institute, Amsterdam, The Netherlands

7. John Hunter Hospital, Newcastle, Australia

LND = lymph node dissection; $L N=$ lymph nodes

* Any $\mathrm{cN} 1$ nodes detected on imaging outside these boundaries prior to surgery were additionally resected.

Figure 1

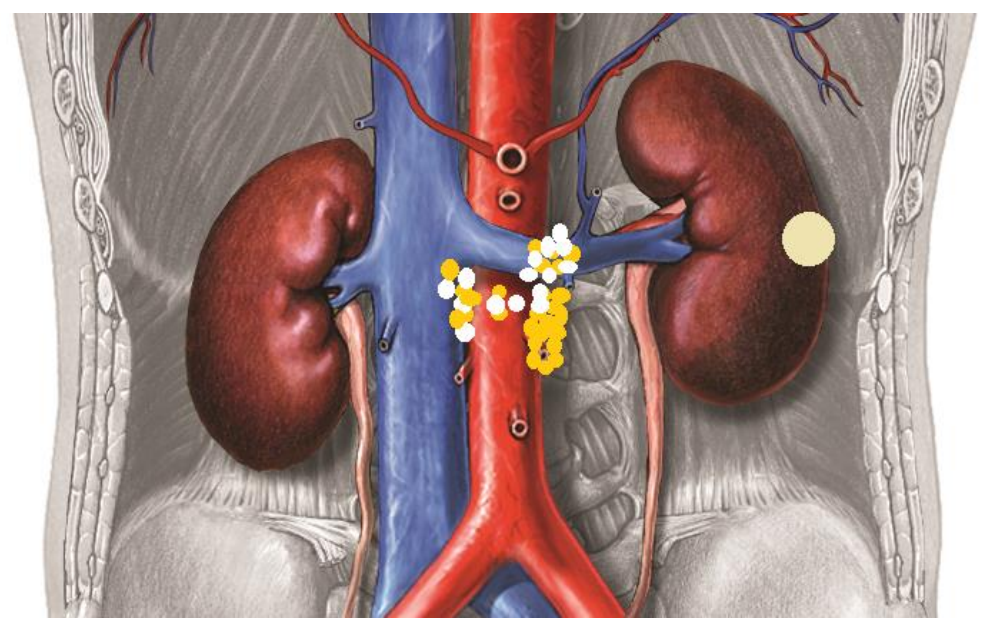




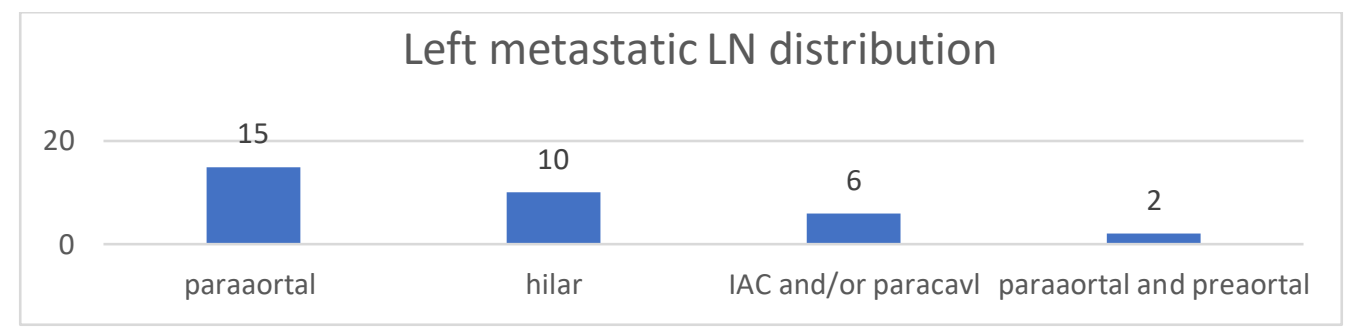

Legend: Left renal cancer sites of cNO (white) and cN1 (yellow) metastatic lymph nodes. Some patients had several lymph nodes and the dots represent lymph nodes, therefore there are more dots than represented in the table. Yellow lymph nodes without filling are dorsally located, retrocaval lymph nodes. Bar chart showing pN1 metastatic LN locations on left side.

Figure 2
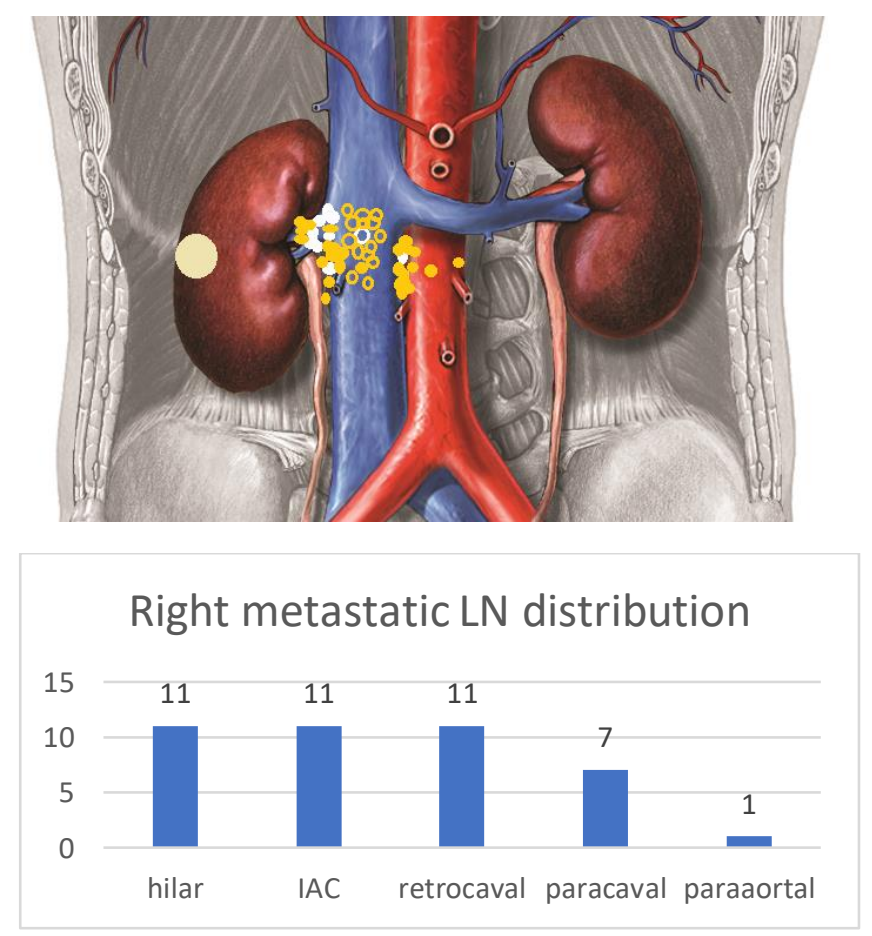

Legend: Right renal cancer sites of cNO (white) and cN1 (yellow) metastatic lymph nodes. Some patients had several lymph nodes and the dots represent lymph nodes, therefore there are more dots than represented in the table. Yellow lymph nodes without filling are dorsally located, retrocaval lymph nodes. Bar chart showing pN1 metastatic LN locations on right side. 


\section{CHAPTER 6}

An analysis of SPECT/CT non-visualization of sentinel lymph nodes in renal tumors

Teele Kuusk, Maarten L. Donswijk, Renato A. Valdés Olmos, Roderick E. De Bruijn, Oscar R. Brouwer, Kees Hendricksen, Simon Horenblas, Katarzyna Jóźwiak, Warner Prevoo, Henk G. Van Der Poel, Bas W. G. Van Rhijn,Esther M. Wit, and Axel Bex

EJNMMI Res. 2018 Dec;8(1):105. 


\section{ABSTRACT}

\section{Background}

Sentinel lymph node biopsy (SLNB) after intratumoral injection of $99 \mathrm{mTc}$ labeled nanocolloid and imaging with scintigraphy and SPECT/CT in renal tumors is feasible. However, sentinel lymph node (SN) non-detection rate with scintigraphy and SPECT/CT is high. The aim of the study was to determine factors affecting non-visualization (NV) of SN imaging in renal tumors. Seventy-eight patients with cT1-3 renal tumors received intratumoral injection of $225 \mathrm{MBq} 99 \mathrm{mTc}$-labeled nanocolloid 1 day before (partial) nephrectomy. Radiotracer injection was followed by anterioposterior and lateral scintigraphy in combination with SPECT/CT 20 min and 2-4 h after. Surgical treatment of the tumor with sentinel lymph node biopsy by aid of $\psi$-probe and-camera was performed the next day. Scintigraphy and SPECT/CT images were evaluated and patient, tumor, and procedure characteristics were collected for 73 eligible patients used in uni- and multivariable analysis of a potential association with NV.

\section{Results}

A total of 80 (mean 1.1, IQR 0-2, max 6) sentinel lymph nodes in 46 patients were detected with scintigraphy and SPECT/CT. Preoperative visualization rate and intraoperative detection rate was $63 \%$ [95\% $\mathrm{Cl} 50-73 \%$ ] and $61 \%$ [95\% $\mathrm{Cl} 49-72 \%]$, respectively. In uni- and multivariable analysis, the only factor associated with non-visualization was age, showing higher odds of non-visualization with higher age.

\section{Conclusion}

Our study demonstrated that non-visualization of SNs in renal tumors is relatively high and is associated with patient age. Furthermore, kidneys and also its tumors are highly vascularized which may cause a wash-out effect that could be identified with decreased kidney-liver ratios. However, in our data, the effect was statistically inconclusive. Further studies are needed to improve visualization and standardize the procedure of SLNB in renal tumors. The percentage of NV limits the use of SLNB for research and clinical purposes in renal cancer. 


\section{Background}

Despite low incidence of lymph node (LN) metastases in renal cancer (RCC), dissemination into lymph nodes portends extremely poor prognosis $[1,2]$. In low risk and high risk cancers, the detection rate with a routine pathohistological staining is only $4 \%$ and $10 \%$, respectively and lymph node dissection (LND) in low risk cancers has no proven survival benefit $[3,4]$. Furthermore, lymphatic drainage in renal tumors is unpredictable and drainage outside local retroperitoneal lymph nodes may be one of the reasons for the lack of a survival advantage with conventional LND [5-7]. Sentinel lymph node biopsy (SLNB) typically consists of a preoperative lymphoscintigraphy to indicate the anatomical location of the first tumor-draining lymph nodes, i.e., the sentinel node(s), combined with a $\mathrm{\gamma}$-probe guided surgical biopsy of these nodes. SLNB may improve staging of RCC especially in patients who have aberrant drainage within or outside retroperitoneal lumbar lymph node basins and might have a role in future translational research of tumor immunology and biology of early metastasis. It has been suggested in prostate cancer that extended pelvic lymph node dissection combined with SLNB increases the yield of nodal lymph node metastases, especially in high risk disease [8]. Due to proven efficacy in staging procedures in many cancer types and less associated morbidity of SLNB compared to LND, SLNB is routinely used in melanoma, breast, head-neck, vulvar, cervical, and penile cancer and actively studied in many other tumor types $[9,10]$. Feasibility of SLNB procedure in renal tumors has been studied and confirmed earlier by our group and others [11, 12]. However, both groups had high rate of non-visualization of sentinel lymph node (SN) on scintigraphy and single-photon emission computed tomography with computed tomography (SPECT/CT). Also, the procedure is currently lacking standardization. These factors may hamper widespread applicability and adoption to further study its use in clinical practice. Therefore, the aim of our study was to determine incidence and predictive factors of non-visualization of SN on scintigraphy and SPECT/CT in renal tumors, evaluate detection rate at surgery, and propose a standardized protocol for future studies.

\section{Methods}

Patients

From 2008 to 2017, 78 patients were enrolled into a feasibility and a single-arm phase II prospective study to investigate lymphatic drainage and the distribution of SN in renal tumors (N06SNR and N08SNR registered under NL26406.031.08 Fig. 1). The studies had medical ethics committee approval and all patients signed written informed consent. Inclusion criteria were CT-based cT1-3 renal tumors $\leq 10 \mathrm{~cm}$ of any subtype, and clinically and radiologically non-metastatic disease (cNOcM0), assessed with pelvic, abdominal, and thoracic contrast-enhanced $\mathrm{CT}$, age $>18$ years, life expectancy $>3$ months, WHO performance status $0-1$, and no prior systemic therapy. Primary endpoint was the percentage of SNs located at any site outside the left or right locoregional retroperitoneal template (LRT) on lymphoscintigraphy and subsequent SPECT/CT imaging as described below. The sample size of the phase II study was based on a Simon two-stage design including 40 patients with SN on SPECT/CT imaging in the final analysis. Patients without visualization of SN were recorded to analyze the failure rate. For this analysis, our objective was to assess the rate and factors contributing to nonvisualization in both studies. 
Sentinel node imaging

One day prior to surgery, a dose of approximately $225 \mathrm{MBq}$ of $99 \mathrm{mTc}$ nanocolloid (Nanocoll C; GE Healthcare, Eindhoven, Netherlands) in a volume of $0.4 \mathrm{ml}$ was injected percutaneously under ultrasound or CT guidance into the tumor. Primary tumors $\leq 4 \mathrm{~cm}$ were injected centrally with a volume of $0.4 \mathrm{ml}$ and $4-10 \mathrm{~cm}$ tumors were injected with 2-3 depots of $0.4 \mathrm{ml}$ around the center avoiding necrotic areas. Following injection, lymphoscintigraphy based on anterioposterior and lateral 5-min planar static images after $20 \mathrm{~min}$ and $2-4 \mathrm{~h}$ was acquired. Subsequently, SPECT/CT was acquired using a hybrid system (SymbiaT, Siemens, Erlangen, Germany). After correction for scatter and tissue attenuation, SPECT and CT images were fused. Multiplanar reconstruction enabled comparison of fused images with concomitant CT images to determine the anatomical location of the SNs (Osirix Dicom viewer with medical imaging software, Pixmeo, Geneva, Switzerland). The nodes draining directly from the tumor on planar lymphoscintigraphy were considered to be SNs and were localized using SPECT/CT. In case of multiple visualized lymph nodes in a basin, the early appearing nodes were considered to be the SNs. Aspects related to tracer injection and activity (spillage, anterior/posterior injection, number of depots, properly injected depot according to SPECT/CT image, kidney/liver activity, depot location) were prospectively evaluated from lymphoscintigraphy and SPECT/CT images by an experienced nuclear medicine physician. Data on injected radiotracer dosage, number of injections, and modality of injection (US or CT) were collected retrospectively from patients' electronic files. All other factors as described in Tables 1 and and 2 were retrieved from medical records.

Sentinel lymph node biopsy

The following day, surgical treatment of the primary tumor combined with SN mapping was performed. In both studies, the boundaries of the locoregional templates were defined as described previously $[5,13]$. Surgical approach (open, laparoscopic, robot-assisted) was decided per case depending on the primary tumor. At surgery, SNs were located in the areas indicated by preoperative SPECT/CT images and detected intraoperatively with a $p$-probe (Neoprobe, Johnson\&Johnson Medical, Hamburg, Germany) in combination with a portable $\gamma$-camera (Sentinella, S102, GEM imaging, Valencia, Spain). Harvested SNs were also measured ex situ with both $\gamma$-probe and $\gamma$ camera. After SN excision, the surgical area was scanned using the portable $\gamma$-camera to verify complete SN removal. For ethical reasons, only SNs accessible through the chosen surgical approach were removed. Sentinel nodes were formalin fixed, paraffin embedded, and cut into $3 \mu \mathrm{m}$ sections according to our institute SLNB protocol. Paraffin sections were stained and examined with hematoxylin and eosin.

Non-sentinel lymph node dissection

Additionally, non-SNs within the retroperitoneal lymph node dissection template were resected for further standard hematoxylin-eosin staining. 


\section{Statistical analysis}

Patient and SLNB procedure characteristics were analyzed with descriptive statistics. Factors associated with the $\mathrm{SN}$ visualization were analyzed using a logistic regression. The analysis included patient data, such as age, gender, BMI (body mass index); tumor characteristics such as tumor size, PT stage, side, polarity, RENAL score, which categorizes renal masses by complexity for surgical decision making, Leibovich score, which predicts metastases free survival after surgical therapy, tumor posteriorly located (yes/no), histology, lymphovascular invasion, necrosis on imaging, necrosis in histology; and procedural and injection techniques characteristics such as volume of the isotope, number of injections, spillage on imaging, defined by a spillage of the tracer $>25 \%$ outside of the tumor seen on SPECT/CT images, depot properly injected, defined as having a depot of the tracer $>75 \%$ inside the tumor (yes/no), depot located anteriorly (yes/no), whereas kidney was divided anterior and posterior on coronal plane at the level of hilar vessels, to determine the complexity of injection because injection anteriorly is more challenging, actual time between injection and early scintigraphic imaging, time between injection and late scintigraphic imaging, kidney/liver activity ratio (calculated by drawing a region of interest (ROI) over the depot in the kidney, then dividing the maximum number of counts in this ROI by the maximum number of counts in a second ROI over the liver) at both early and late planar scintigraphy as a potential indicator of wash-out of the tracer; and outcome parameters such as any distant metastases (yes/no), death (yes/no) (Table 1). Additional logistic regression was performed with age categories split at median age of the patients to test whether this cut-off can be used to define a group of patients with a non-visualization. For testing wash-out association with non-visualization, we calculated the ratio (activity in the kidney) / (activity in the liver), whereas higher grades of radiotracer wash-out decrease this ratio. P value $\leq 0.05$ was considered statistically significant and all tests were two-sided. Odds ratios are presented with their $95 \%$ confidence intervals $(\mathrm{Cl})$. Factors for which $p$ value reached $<0.1$ in a univariable analysis were included in a multivariable model. Statistical analyses were performed using SPSS version 22 (IBM, Chicago, IL, USA).

\section{Results}

Of the 78 patients, 5 patients were excluded because of ineligibility. Therefore, 73 patients were available for final analysis (Fig. (Fig.1).1). The characteristics of the patients and the procedure are shown in Table 2. The majority of patients (68.5\%) had low or intermediate risk clear cell RCC. Median time between the injection and early and late imaging with visualization and nonvisualization was $33 \mathrm{~min}$ (IQR 19-51 $\mathrm{min}$ ), $28 \mathrm{~min}$ (IQR 15-65 min) and $149 \mathrm{~min}$ (IQR 175-188), 182 min (IQR 139-194) respectively. Median tracer dose was 209 mBq (IQR 187-222) and 212 (IQR 196218) with visualization and non-visualization, respectively. Visualization of SNs on imaging was $63 \%$ [95\% $\mathrm{Cl} 50-73 \%$ ]. All SNs visualized on planar images were also visualized on SPECT/CT. A total of 80 SNs in 46 patients were visualized, and the mean number of SNs on imaging was 1.1 (IQR 0-2).

In six patients, no SN could be harvested. In three patients, there was no activity detected with a $\mathrm{Y}$ probe in vivo nor ex vivo and a selective dissection of lymph nodes was performed in the area of the SNs as visualized on SPECT/CT. In three patients, radioactive SNs were detected with a $\gamma$-probe but these were not harvested due to severe obesity and risky dissection or non-accessibility through the exposure. On the contrary, in five patients with non-visualization on pre-operative imaging, 
radioactive SNs were detected with a $\mathrm{\gamma}$-probe the next day at surgery. Therefore, SNs were detected and harvested in 45 patients $(61 \%$ [95\% $\mathrm{Cl} 49-72 \%])$. The mean number of harvested SNs was 2.12 (IQR 0-3) and mean number of non-SNs was 3.01 (IQR 0-4). A total of 155 SNs were removed. Two patients had occult SN metastases (one was with visualized SN and the other had non-visualization).

Factors associated with non-visualization

Table 1 shows factors for visualization, univariable and multivariable analysis. In univariable and multivariable analysis, an increased risk of non-visualization was associated with patient age, showing a trend toward older patients having higher risk of non-visualization ( $p=0.008$ and 0.002 , respectively). Whereas including age categories with below and above median age of 59 years did not show statistical significance in univariable analysis $(p=0.11)$. Other factor associated with nonvisualization in univariable analysis was kidney/liver activity on scintigraphy at late planar imaging; however, this factor did not reach statistical significance in multivariable model.

\section{Discussion}

SLNB in general has a role in multiple tumors in studying lymphatic drainage as well as having importance in staging with an advantage of being a more sensitive and less aggressive procedure than LND [9, 14-17]. SLNB has proven efficacy in breast, head-neck, vulvar, cervical and penile cancer, and melanoma and is actively studied in many other tumor types [9, 18, 19]. The first feasibility and outcome studies of SLNB in renal tumors have been published showing that the procedure is safe; however, further studies in a larger cohort for clinical or research utility in high risk cancer may be limited due to a significant rate of non-visualization. Two groups that studied feasibility of renal SN with lymphoscintigraphy and SPECT/CT reported non-visualization rates varying between 25 and 73\% [11, 12]. Although inclusion criteria, timing of imaging, imaging, and mapping techniques were comparable, distinct methods for radiotracer injection were used and also radiotracer dose, imaged area, and modalities for SN detection varied (Additional file 1: Table S1). A few factors of Sherif et al.'s group that could theoretically have lowered their visualization rate were low volume of injected radiotracer and imaging only the abdominal area, whereas our group included thoracic lymphoscintigraphy, where $20 \%$ of SNs were found. Although, Sherif et al. also used blue dye intraoperatively, they detected only $12.5 \%$ of SN with this technique, which is in line with earlier reported significantly lower sensitivity of blue dye (50-70\%) compared to radiotracer [12, 20]. Today, our study has the largest renal tumor SLNB cohort with 73 patients presenting $37 \%$ of non-visualization on scintigraphy and SPECT/CT imaging with intratumoral injection. In the study of Sherif et al. [12] consisting of 13 patients, the radioisotope was injected peritumorally. Nonvisualization on scintigraphy and SPECT/CT was reported in 8 out of 11 (73\%) of cases. They detected most of the SNs using a $y$-probe, whereas in our study most of the SNs were visualized by scintigraphy combined with SPECT/CT, and the minority was detected with $\gamma$-probe only ( $n=5(18 \%)$. Compared to SLNB in other primary tumor sites, which have scintigraphy and SPECT/CT visualization rates of $82-100 \%$, SLN visualization in renal tumors is lower $[9,21]$.

It has been suggested in other tumor types that non-visualization of lymphatic drainage can be related to patient age, BMI, size of the tumor, high nodal tumor load, neoadjuvant therapy, occlusion of lymphatics, radioisotope type, dose and volume, and lymphovascular invasion [9, 14-16, 22]. Including all the possible factors (Table 2 ), we acknowledged some specific aspects that might be 
associated with non-visualization regarding kidney physiology. Due to high vascularity of the kidney and vascular tumors, inappropriate radiotracer injection into a highly vascularized area of the renal tumor may cause a wash-out phenomenon which might be related to non-visualization. However, our results were not conclusive about the association between non-visualization and wash-out phenomena in multivariable analysis.

The only aspect which demonstrated association with non-visualization in our group was age with a tendency toward having more non-visualization with older age. Age has been associated with false negative rate in melanoma and breast cancer patients and it has been speculated that it may be caused by degeneration of the lymphatic system resulting in decreased rate of lymph flow with increasing age or secondary to variable or sluggish lymphatics in older patients [14, 23-25]. In fact, change in lymphatic function of SNs in older patients and lymphatic aging have been demonstrated earlier $[14,25]$. This has been shown by decline in radiotracer transit with older age in melanoma patients with intraoperative lower counts of radioactivity measured with a $\gamma$-probe. In addition, there has also been association between age and lower number of metastatic SNs which further suggests an alteration of lymphatic function with aging [14]. However, due to lacking records of radioactivity count measurements and low number of $\mathrm{SN}$ metastatic cases, further analysis to substantiate an association in our cohort is unavailable.

Other accepted determinants that have shown association with non-visualization in different tumor types are tumor location in the organ and method of injection (intratumorally, peritumorally) [2628]. Comparison of intra-and peritumoral hybrid isotope injection in prostate cancer was studied recently, showing more non-visualization with intratumoral injection than peritumoral; however, more $\mathrm{pN}$ positive patients were detected with intratumoral method [26]. Detection of SNs has also been better with periareolar compared to intratumoral radiotracer injection in breast cancer [29]. However, considering the kidney's high vascularity, these tumor-bearing organs are not comparable and in fact injecting radiotracer into both the kidney and tumor could cause substantial radiotracer wash-out. Aspects of injection, e.g., injection site, number of depots, etc., were not associated with non-visualization in our study; however, it has been described that most of the lymphatics in kidney cancers with sinus vein invasion locate peritumorally and less are seen within the tumors [30]. This would suggest that Sherif et al. with peritumoral injection [12] should have had higher rate of visualization; however, they detected most of the SNs by $\mathrm{\gamma}$-probe only and non-visualization rate with SPECT/CT was even higher than in our series.

Another important consideration regarding radiotracer injection is tumor location in the kidney (anterior, posterior, upper, intermedial, lower pole). Even though our results did not demonstrate difference between the tumor location and visualization, ultrasound-guided intratumoral injection can be technically challenging especially for anterior location. In addition, dynamics of the kidney caused by breathing can challenge the precision of the injection.

Non-visualization could also be related to lymphatic drainage directly into the thoracic duct without interfering retroperitoneal lymph nodes, whereas posterior lumbar lymphatics are more inclined to this route [5, 13, 30-32]. Thoracic duct might be also the path for a lympho-venous connection and subsequent hematogenic metastases mainly into lungs which is one of the most common metastatic sites in renal cancer and may occur without concurrent retroperitoneal lymph node metastatic involvement. An indication for the alternative drainage from the posterior lumbar lymphatics directly into the thoracic duct would be an association of non-visualization with posterior radiotracer depots, 
multiple injections, or larger tumors, because in all these occasions probability of radiotracer drainage into the posterior lumbar lymphatics are theoretically higher [13]. However, due to methodology used, we were not able to confirm this in our study. We were also unable to confirm the route on imaging in the cases with non-visualization because scintigraphy and SPECT/CT imaging probably are not sensitive enough to detect minimal amounts of radioactivity in the thoracic duct.

In view of the experience from the studies performed by the two different groups [11, 12], it appears that the majority of SNs are visualized 2-4 $\mathrm{h}$ after tracer injection and in case of non-visualization, delayed imaging can be considered. Imaging should consist of planar scintigraphy of the trunk including the thoracic cavity because $20 \%$ of sentinel lymph nodes are visualized in this area [5], combined with SPECT/CT of the area of interest. Intraoperative SN detection rates with $\gamma$-probe and camera were comparable to preoperative lymphoscintigraphy and SPECT/CT, indicating that timing between imaging and operation in our study was optimal. $99 \mathrm{mTc}$ nanocolloid was used as a radiotracer in both studies; however, whether its combination with ICG could improve intraoperative detection rate with an impact to overall detection rate and also reduce the time spent on SN harvesting remains to be studied. Concerning the dosage, according to depot activity measurements, $225 \mathrm{MBq} 99 \mathrm{mTc}$ appears to be adequate. Injection site peri- or intratumorally is debatable; however, we believe that injection into periphery of the tumor avoiding necrotic areas could result in better distribution of the tracer because high vascularization of the kidneys may cause a wash-out phenomenon. Nevertheless, when high-risk tumors are studied, additional peritumoral injection can be considered due to a more peri- than intratumoral lymphatic distribution [30].

Our study has a number of limitations. We enrolled mostly low risk patients, who have low incidence of lymph node metastatic disease. In addition, the study was not primarily designed to detect nonvisualization, thus it has the limitations inherent to any retrospective data analysis. Besides, this study is not able to explain a predominant cause for non-visualization. Finally, the number of patients is low which may have an effect on the overall statistical power in our study.

\section{Conclusion}

Non-visualization with scintigraphy and SPECT/CT in renal tumors is high and older age is the only factor associated with non-visualization. Determining whether peritumoral radiotracer injection in high-risk tumors or hybrid radiotracer with ICG could improve the detection rate requires another prospective study with primarily detection rate as a primary endpoint.

\section{Acknowledgements}

We are grateful for Antoni Van Leeuwenhoek Netherlands Cancer Institute team members for their contribution to the study.

\section{Funding None.}

\section{Abbreviations}

LN Lymph node

LND Lymph node dissection 
RCC Renal cancer

SLNB Sentinel lymph node biopsy

SN Sentinel lymph node

SPECT/CT Single-photon emission computed tomography with computed tomography

\section{Ethics approval and consent to participate}

All procedures performed in studies involving human tissue were in accordance with the ethical standards of the institutional and/or national research committee and with the principles of the 1964 Declaration of Helsinki and its later amendments or comparable ethical standards.

\section{Consent for publication}

All authors read the manuscript and consented for its publication.

\section{Competing interests}

The authors declare that they have no competing interests.

\section{References}

1. Gershman B, Moreira DM, Thompson RH, Boorjian SA, Lohse CM, Costello BA, Cheville JC, et al. Renal cell carcinoma with isolated lymph node involvement: long-term natural history and predictors of oncologic outcomes following surgical resection. Eur Urol. 2017;72:300-306. doi: 10.1016/j.eururo.2016.12.027.

2. Blute ML, Leibovich BC, Cheville JC, Lohse CM, Zincke H. A protocol for performing extended lymph node dissection using primary tumor pathological features for patients treated with radical nephrectomy for clear cell renal cell carcinoma. J Urol. 2004;172:465-469. doi: 10.1097/01.ju.0000129815.91927.85.

3. Blom JHM, Van Poppel H, Maréchal JM, Jacqmin D, Schröder FH, de Prijck L, et al. Radical nephrectomy with and without lymph-node dissection: final results of European Organization for Research and Treatment of Cancer (EORTC) randomized phase 3 trial 3088. Eur Urol. 2009;55:28-34. doi: 10.1016/j.eururo.2008.09.052.

4. Tilki D, Chandrasekar T, Capitanio U, Ciancio G, Daneshmand S, Gontero P, et al. Impact of lymph node dissection at the time of radical nephrectomy with tumor thrombectomy on oncological outcomes: results from the International renal cell carcinoma-venous Thrombus consortium (IRCCVTC) Urol Oncol. 2018;36(2):79.e11-79.e17. doi: 10.1016/j.urolonc.2017.10.008.

5. Kuusk T, De Bruijn R, Brouwer RO, De Jong J, Donswijk M, Grivas N, et al. Lymphatic drainage from renal tumors in vivo: a prospective sentinel node study using SPECT/CT imaging. J Urol. 2018;199(6):1426-1432. doi: 10.1016/j.juro.2017.11.112.

6. Moschini M, Dell'Oglio P, Larcher A, Capitanio U. Lymph node dissection for renal cell carcinoma: what are we missing? Curr Opin Urol. 2016;26(5):424-431. doi: 10.1097/MOU.0000000000000312. 
7. John NT, Blum KA, Hakimi AA. Role of lymph node dissection in renal cell cancer. Urol Oncol. 2018. 10.1016/j.urolonc.2018.03.006.

8. Wit EMK, Acar C, Grivas N, Yuan C, Horenblas S, Liedberg F, et al. Sentinel node procedure in prostate cancer: a systematic review to assess diagnostic accuracy. Eur Urol. 2017;71(4):596-605. doi: 10.1016/j.eururo.2016.09.007.

9. INTERNATIONAL ATOMIC ENERGY AGENCY . Guided intraoperative Scintigraphic tumour targeting (GOSTT), IAEA human health series no. 29. Vienna: IAEA; 2014. pp. 67-262.

10. Brouwer OR, van der Poel HG, Bevers RF, van Gennep EJ, Horenblas S. Beyond penile cancer, is there a role for sentinel node biopsy in urological malignancies? Clin Transl Imaging. 2016;4(5):395410. doi: 10.1007/s40336-016-0189-4.

11. Bex A, Vermeeren L, de Windt G, Prevoo W, Horenblas S, Olmos RA. Feasibility of sentinel node detection in renal cell carcinoma: a pilot study. Eur J Nucl Med Mol Imaging. 2010;37:1117-1123. doi: 10.1007/s00259-009-1359-7.

12. Sherif AM, Eriksson E, Thörn M, Vasko J, Riklund K, Ohberg L, Ljungberg BJ. Sentinel node detection in renal cell carcinoma. A feasibility study for detection of tumour-draining lymph nodes. BJU Int. 2012;109(8):1134-1139. doi: 10.1111/j.1464-410X.2011.10444.x.

13. Karmali RJ, Suami H, Wood CG, Karam JA. Lymphatic drainage in renal cell carcinoma: back to the basics. BJU Int. 2014;114(6):806-817. doi: 10.1111/bju.12814.

14. Conway WC, Faries MB, Nicholl MB, Terando AM, Glass EC, Sim M, et al. Age-related lymphatic dysfunction in melanoma patients. Ann Surg Oncol. 2009;16(6):1548-1552. doi: 10.1245/s10434009-0420-x.

15. Brenot-Rossi I, Houvenaeghel G, Jacquemier J, Bardou VJ, Martino M, Hassan-Sebbag N, Pasquier J. Nonvisualization of axillary sentinel node during lymphoscintigraphy: is there a pathologic significance in breast cancer? J Nucl Med. 2003;44(8):1232-1237.

16. Kroon BK, Valdes Olmos R, Nieweg OE, Horenblas S. Non-visualization of sentinel lymph nodes in penile carcinoma. Eur J Nucl Med Mol Imaging. 2005;32:1096-1099. doi: 10.1007/s00259-005-1822$z$.

17. Madu MF, Wouters MW, van Akkooi AC. Sentinel node biopsy in melanoma: current controversies addressed. Eur J Surg Oncol. 2017;43(3):517-533. doi: 10.1016/j.ejso.2016.08.007.

18. Acar C, Kleinjan GH, van den Berg NS, Wit EM, van Leeuwen FW, van der Poel HG. Advances in sentinel node dissection in prostate cancer from a technical perspective. Int J Urol. 2015;22:898-909. doi: 10.1111/iju.12863.

19. Blok JM, Kerst JM, Vegt E, Brouwer OR, Meijer RP, Bosch JLHR, et al. Sentinel Node Biopsy in Clinical Stage I Testicular Cancer Enables Early Detection of Occult Metastatic Disease. BJU Int. 2018;10.1111/bju.14618.

20. Brouwer OR, van den Berg NS, Matheron HM, van der Poel HG, van Rhijn BW, Bex A, et al. A hybrid radioactive and fluorescent tracer for sentinel node biopsy in penile carcinoma as a potential replacement for blue dye. Eur Urol. 2014;65(3):600-609. doi: 10.1016/j.eururo.2013.11.014. 
21. Vermeeren L, Muller SH, Meinhardt W, Valdes Olmos RAE. Optimizing the colloid particle concentration for improved preoperative and intraoperative image-guided detection of sentinel nodes in prostate cancer. J Nucl Med Mol Imaging. 2010;37(7):1328-1334. doi: 10.1007/s00259-0101410-8.

22. Leijte JA, Kroon BK, Valdes Olmos RA, Nieweg OE, Horenblas S. Reliability and safety of current dynamic sentinel node biopsy for penile carcinoma. Eur Urol. 2007;52:170-177. doi: 10.1016/j.eururo.2007.01.107.

23. Sinnamon AJ, Neuwirth MG, Bartlett EK, Zaheer S, Etherington MS, Xu X, et al. Predictors of false negative sentinel lymph node biopsy in trunk and extremity melanoma. J Surg Oncol. 2017;116(7):848-855. doi: 10.1002/jso.24743.

24. Tanis PJ, van Sandick JW, Nieweg OE, Valdés Olmos RA, Rutgers EJ, Hoefnagel CA, et al. The hidden sentinel node in breast cancer. Eur J Nucl Med Mol Imaging. 2002;29:305-311. doi: 10.1007/s00259-001-0732-y.

25. Luscieti P, Hubschmid T, Cottier H, Hess MW, Sobin LH. Human lymph node morphology as a function of age and site. J Clin Pathol. 1980;33(5):454-461. doi: 10.1136/jcp.33.5.454. [PMC free article]

26. Wit E, Kleinjan G, Donswijk M, Leeuwen Van F, Van Der Poel H. The influence of tracer injection into the prostate for the detection of lymph node metastases in prostate cancer during robot assisted radical prostatectomy with sentinel node procedure: a phase II randomized comparison. Eur Urol Suppl. 2018;17(2):e1161. doi: 10.1016/S1569-9056(18)31648-8.

27. KleinJan G. H., van Werkhoven E., van den Berg N. S., Karakullukcu M. B., Zijlmans H. J. M. A. A., van der Hage J. A., van de Wiel B. A., Buckle T., Klop W. M. C., Horenblas S., Valdés Olmos R. A., van der Poel H. G., van Leeuwen F. W. B. The best of both worlds: a hybrid approach for optimal pre- and intraoperative identification of sentinel lymph nodes. European Journal of Nuclear Medicine and Molecular Imaging. 2018;45(11):1915-1925. doi: 10.1007/s00259-018-4028-x.

28. Bodurtha Smith AJ, Fader AN, Tanner EJ. Sentinel lymph node assessment in endometrial cancer: a systematic review and meta-analysis. Am J Obstet Gynecol. 2017;216(5):459-476.e10. doi: 10.1016/j.ajog.2016.11.1033.

29. Garcia-Manero M, Olartecoechea B, Royo P. Different injection sites of radionuclide for sentinel lymph node detection in breast cancer: single institution experience. Eur J Obstet Gynecol Reprod Biol. 2010;153(2):185-187. doi: 10.1016/j.ejogrb.2010.06.024.

30. Bonsib SM. Renal lymphatics, and lymphatic involvement in sinus vein invasive (pT3b) clear cell renal cell carcinoma: a study of 40 cases. Mod Pathol. 2006;19(5):746-753. doi: 10.1038/modpathol.3800589.

31. Brouwer OR, Noe A, Olmos RA, Bex A. Lymphatic drainage from renal cell carcinoma along the thoracic duct visualized with SPECT/CT. Lymphat Res Biol. 2013;11:233-238. doi:

10.1089/Irb.2013.0017.] 
32. Bex A, Vermeeren L, Meinhardt W, Prevoo W, Horenblas S, Valdés Olmos RA. Intraoperative sentinel node identification and sampling in clinically node-negative renal cell carcinoma: initial experience in 20 patients. World J Urol. 2011;29:793-799. doi: 10.1007/s00345-010-0615-6. 
Diagram 1. Consort diagram for study participants

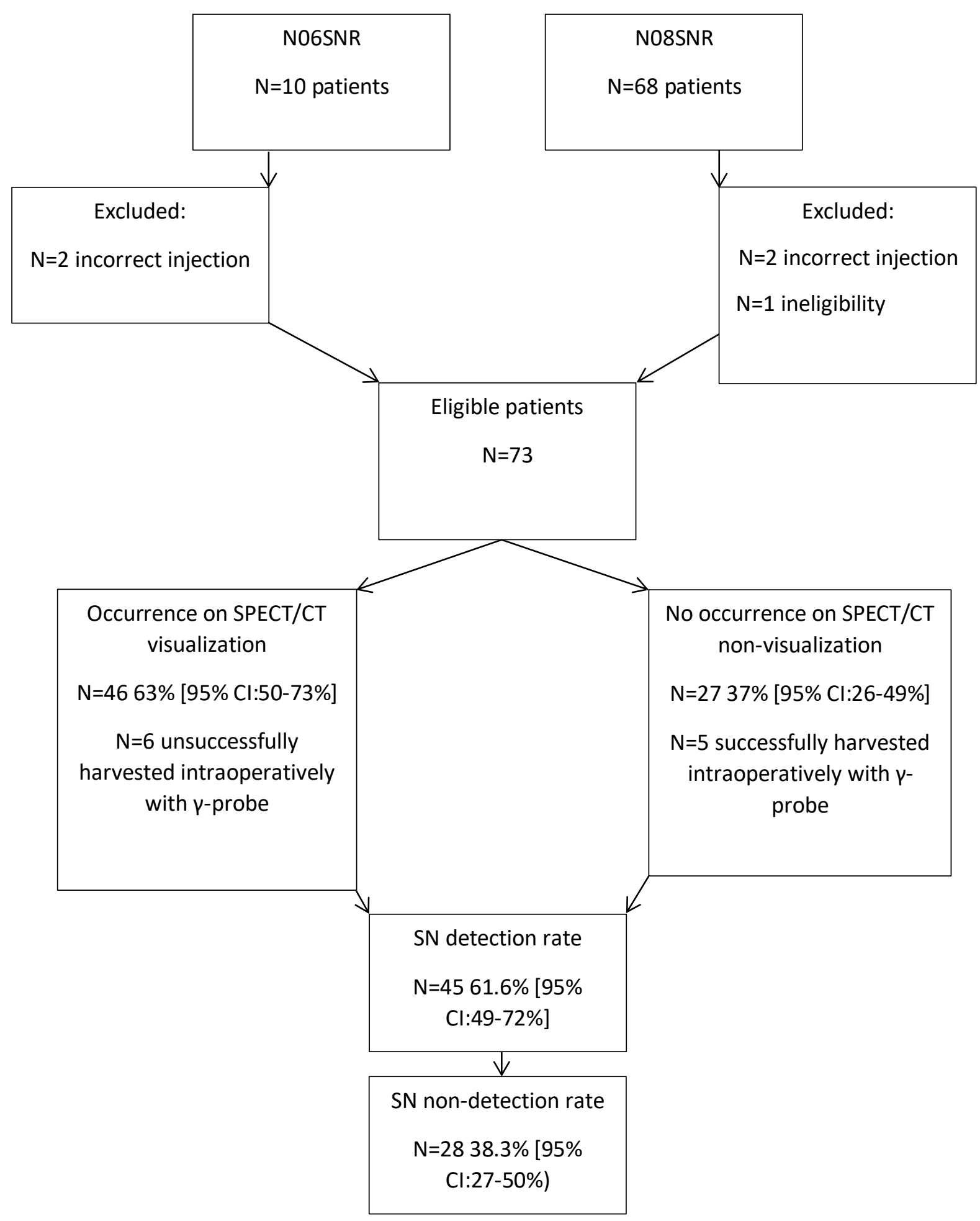


Table 1. Factors for visualization, univariable and multivariable analysis

\begin{tabular}{|c|c|c|c|c|}
\hline & Univariable analy & & Multivariable & analysis \\
\hline Factor & OR [95\% Cl] & $\begin{array}{l}P \\
\text { value }\end{array}$ & OR $[95 \% \mathrm{Cl}]$ & $P$ value \\
\hline $\begin{array}{l}\text { Number of } \\
\text { patients }\end{array}$ & 73 & & & \\
\hline Age (years) & $0.92[0.87-0.97]$ & 0.008 & $\begin{array}{l}0.88 \text { [0.81- } \\
0.95]\end{array}$ & 0.002 \\
\hline $\begin{array}{l}\text { Age above } 59 \\
\text { (years) } \\
\text { Yes } \\
\text { No }\end{array}$ & $\begin{array}{l}1.0 \\
0.44[0.15-1.22]\end{array}$ & 0.11 & & \\
\hline $\begin{array}{l}\text { Gender } \\
\text { Female } \\
\text { Male }\end{array}$ & $\begin{array}{l}1.0 \\
0.85[0.32-2.20]\end{array}$ & 0.74 & & \\
\hline BMI & 0.99 [0.92-1.06] & 0.99 & & \\
\hline $\begin{array}{l}\text { Tumour size } \\
\text { (cm) }\end{array}$ & 0.89 [0.71-1.12] & 0.12 & & \\
\hline $\begin{array}{l}\text { Side } \\
\text { Right } \\
\text { Left }\end{array}$ & $\begin{array}{l}1.0 \\
0.92[0.35-2.40]\end{array}$ & 0.87 & & \\
\hline $\begin{array}{l}\text { Polarity } \\
\text { Upper pole } \\
\text { Intermedial pole } \\
\text { Lower pole }\end{array}$ & $\begin{array}{l}1.0 \\
2.35[0.53-10.4] \\
0.71[0.24-2.09]\end{array}$ & $\begin{array}{l}0.25 \\
0.53\end{array}$ & & \\
\hline $\begin{array}{l}\text { Tumour } \\
\text { location } \\
\text { Posterior } \\
\text { Yes } \\
\text { No }\end{array}$ & $\begin{array}{l}1.0 \\
0.65[0.25-1.70]\end{array}$ & 0.38 & & \\
\hline $\begin{array}{l}\text { RENAL score } \\
\text { Low } \\
\text { Moderate } \\
\text { High }\end{array}$ & $\begin{array}{l}1.0 \\
0.75[0.20-2.77] \\
0.84[0.27-2.62]\end{array}$ & $\begin{array}{l}0.66 \\
0.76\end{array}$ & & \\
\hline $\begin{array}{l}\text { pT stage } \\
\text { T1a } \\
\text { T1b } \\
\text { T2a } \\
\text { T2b } \\
\text { T3a }\end{array}$ & $\begin{array}{l}1.0 \\
2.7[0.33-21.9] \\
0.91[0.19-4.35] \\
0.60[0.08-4.40] \\
1.20[0.07-19.6]\end{array}$ & $\begin{array}{l}0.35 \\
0.91 \\
0.61 \\
0.89\end{array}$ & & \\
\hline $\begin{array}{l}\text { ccRCC } \\
\text { Yes }\end{array}$ & 1.0 & & & \\
\hline
\end{tabular}




\begin{tabular}{|c|c|c|c|c|}
\hline No & $0.42[0.13-1.33]$ & 0.14 & & \\
\hline $\begin{array}{l}\text { Non ccRCC } \\
\text { Yes } \\
\text { No }\end{array}$ & $\begin{array}{l}1.46[0.44- \\
4.86] \\
1.0\end{array}$ & 0.53 & & \\
\hline $\begin{array}{l}\text { Lymphovascular } \\
\text { invasion } \\
\text { Yes } \\
\text { No }\end{array}$ & $\begin{array}{l}1.0 \\
1.19[0.20-6.97]\end{array}$ & 0.84 & & \\
\hline $\begin{array}{l}\text { Necrosis on } \\
\text { imaging } \\
\text { Yes } \\
\text { No }\end{array}$ & $\begin{array}{l}1.0 \\
0.41[0.10-1.72]\end{array}$ & 0.22 & & \\
\hline $\begin{array}{l}\text { Necrosis in } \\
\text { histology } \\
\text { Yes } \\
\text { No }\end{array}$ & $\begin{array}{l}1.0 \\
0.42[0.16-1.12]\end{array}$ & 0.08 & $\begin{array}{l}1.0 \\
1.63[0.44- \\
6.05]\end{array}$ & 0.46 \\
\hline $\begin{array}{l}\text { Leibovich score } \\
\text { Low } \\
\text { Moderate } \\
\text { High }\end{array}$ & $\begin{array}{l}1.0 \\
0.65[0.16-2.60] \\
0.52[0.12-2.18]\end{array}$ & $\begin{array}{l}0.54 \\
0.37\end{array}$ & & \\
\hline $\begin{array}{l}\text { High risk } \\
\text { Yes } \\
\text { No }\end{array}$ & $\begin{array}{l}0.58[0.13-2.65] \\
1.0\end{array}$ & 0.48 & & \\
\hline Dose of isotope & $0.99[0.97-1.01]$ & 0.83 & & \\
\hline $\begin{array}{l}\text { Number of } \\
\text { injections }\end{array}$ & $1.47[0.57-3.80]$ & 0.46 & & \\
\hline $\begin{array}{l}\text { Spillage } \\
\text { Yes } \\
\text { No }\end{array}$ & $\begin{array}{l}1.0 \\
1.84[0.51-6.55]\end{array}$ & 0.34 & & \\
\hline $\begin{array}{l}\text { Depot properly } \\
\text { injected } \\
\text { Yes } \\
\text { No }\end{array}$ & $\begin{array}{l}1.0 \\
0.96[0.35-2.61]\end{array}$ & 0.93 & & \\
\hline $\begin{array}{l}\text { Anterior depot } \\
\text { Yes } \\
\text { No }\end{array}$ & $\begin{array}{l}1.0 \\
1.15[0.38-3.45]\end{array}$ & 0.79 & & \\
\hline $\begin{array}{l}\text { Time between } \\
\text { injection and } \\
\text { early imaging }\end{array}$ & $1.0[1.0-1.0]$ & 0.69 & & \\
\hline
\end{tabular}




\begin{tabular}{|l|l|l|l|l|}
\hline $\begin{array}{l}\text { Time between } \\
\text { injection and } \\
\text { late imaging }\end{array}$ & $1.0[1.0-1.0]$ & 0.25 & & \\
\hline $\begin{array}{l}\text { Kidney/liver } \\
\text { activity ratio at } \\
\text { early } \\
\text { scintigraphy }\end{array}$ & $1.0[0.99-1.00]$ & 0.08 & $\begin{array}{l}0.98[0.95- \\
1.01]\end{array}$ & 0.45 \\
\hline $\begin{array}{l}\text { Kidney/liver } \\
\text { activity ratio at } \\
\text { late } \\
\text { scintigraphy }\end{array}$ & $1.0[0.99-1.00]$ & 0.05 & $\begin{array}{l}1.02[0.97- \\
1.07]\end{array}$ & 0.37 \\
\hline $\begin{array}{l}\text { Metastases } \\
\text { Yes } \\
\text { No }\end{array}$ & 1.0 & $0.96[0.30-3.05]$ & 0.96 & \\
\hline $\begin{array}{l}\text { Death } \\
\text { Yes } \\
\text { No }\end{array}$ & $0.52[0.22-1.23]$ & 0.13 & & \\
\hline
\end{tabular}

Legend: numbers in bold are statistically significant $p \leq 0.05$. BMI body mass index; $S N$ sentinel node; ccRCC clear cell renal cell carcinoma; OR odds ratio

Table 2. Patient and procedure characteristics for visualization and non-visualization

\begin{tabular}{|l|l|l|}
\hline & $\begin{array}{l}\text { Visualization } \\
\text { N and \% or } \\
\text { median and IQR }\end{array}$ & $\begin{array}{l}\text { Non-visualization } \\
\text { N and \% or } \\
\text { median and IQR }\end{array}$ \\
\hline $\begin{array}{l}\text { Number of } \\
\text { patients }\end{array}$ & $46(63 \%)$ & $27(37 \%)$ \\
\hline $\begin{array}{l}\text { Gender } \\
\text { Memale }\end{array}$ & $22(47.8 \%)$ & $14(51.9 \%)$ \\
\hline $\begin{array}{l}\text { Age, years } \\
\text { (median, IQR) }\end{array}$ & $24(52.2 \%)$ & $13(48.1 \%)$ \\
\hline $\begin{array}{l}\text { Age above 59 } \\
\text { years } \\
\text { Age below 59 } \\
\text { years }\end{array}$ & $21(45.7 \%)$ & $62(54-70)$ \\
\hline $\begin{array}{l}\text { BMI (median, } \\
\text { IQR) }\end{array}$ & $27(23-30)$ & $17(63 \%)$ \\
\hline
\end{tabular}




\begin{tabular}{|c|c|c|}
\hline $\begin{array}{l}\text { Size of the } \\
\text { tumour }(\mathrm{cm}) \\
\text { (median, IQR) }\end{array}$ & $5.6 \mathrm{~cm}(4.1-7.0)$ & $6.5 \mathrm{~cm}(5.0-8.0)$ \\
\hline $\begin{array}{l}\text { Tumour location } \\
\text { Side }\end{array}$ & & \\
\hline Right & 23 (50\%) & 13 (48.1\%) \\
\hline $\begin{array}{l}\text { Left } \\
\text { Polarity }\end{array}$ & $23(50 \%)$ & 14 (51.9\%) \\
\hline Upper pole & $12(26.1 \%)$ & 3 (11.1\%) \\
\hline Intermedial pole & $17(37 \%)$ & 14 (51.9\%) \\
\hline $\begin{array}{l}\text { Lower pole } \\
\text { Axial position }\end{array}$ & $17(37 \%)$ & $10(37.1 \%)$ \\
\hline Posterior & 19 (41.3\%) & 14 (51.9\%) \\
\hline Anterior & 27 (58.7\%) & 13 (48.1\%) \\
\hline \multicolumn{3}{|l|}{ RENAL score } \\
\hline Low risk & 7 (15.2\%) & 5 (18.5\%) \\
\hline Moderate risk & $11(23.9 \%)$ & 7 (25.9\%) \\
\hline High risk & $28(60.9 \%)$ & 15 (55.6\%) \\
\hline \multicolumn{3}{|l|}{ pTstage } \\
\hline T1a & 9 (19.6\%) & $2(7.4 \%)$ \\
\hline T1b & $26(56.5 \%)$ & 17 (63\%) \\
\hline T2a & $4(8.7 \%)$ & $4(14.8 \%)$ \\
\hline $\mathrm{T} 2 \mathrm{~b}$ & $2(4.3 \%)$ & $1(3.7 \%)$ \\
\hline T3a & 5 (10.9\%) & 3 (11.1\%) \\
\hline \multicolumn{3}{|l|}{ pNstage } \\
\hline NO & 44 (95.7\%) & $21(77.8 \%)$ \\
\hline N1 & 1 (2.2\%) & 1 (3.7\%) \\
\hline
\end{tabular}




\begin{tabular}{|c|c|c|}
\hline$N x$ & $1(2.2 \%)$ & 5 (18.5\%) \\
\hline \multicolumn{3}{|l|}{ Histology } \\
\hline $\operatorname{ccRCC}$ & 30 (65.2\%) & 22 (81.5\%) \\
\hline Pap I & 5 (10.9\%) & $2(7.4 \%)$ \\
\hline Pap II & $3(6.5 \%)$ & $1(3.7 \%)$ \\
\hline Chromophobe & $4(8.7 \%)$ & $1(3.7 \%)$ \\
\hline Oncocytoma & $3(6.5 \%)$ & 0 \\
\hline Fibrous tumour & $1(2.2 \%)$ & 0 \\
\hline NOS & 0 & $1(3.7 \%)$ \\
\hline \multicolumn{3}{|l|}{ Fuhrman grade } \\
\hline I & $4(8.9 \%)$ & $4(14.8 \%)$ \\
\hline II & $12(26.7 \%)$ & $12(44.4 \%)$ \\
\hline III & $13(28.9 \%)$ & $3(11.1 \%)$ \\
\hline IV & 0 & 4 (14.8\%) \\
\hline NA & $17(37.8 \%)$ & $4(14.8 \%)$ \\
\hline \multicolumn{3}{|l|}{ Leibovich score } \\
\hline Low & 18 (39\%) & $10(37 \%)$ \\
\hline Intermediate & $13(28.3 \%)$ & 9 (33.3\%) \\
\hline High & $4(8.7 \%)$ & $4(14.8 \%)$ \\
\hline NA & 11 (23.9\%) & $4(14.8 \%)$ \\
\hline $\begin{array}{l}\text { Lymphovascular } \\
\text { invasion } \\
\text { Yes } \\
\text { No }\end{array}$ & $\begin{array}{l}4(8.7 \%) \\
42(91.3 \%)\end{array}$ & $\begin{array}{l}2(7.4 \%) \\
25(92.6 \%)\end{array}$ \\
\hline $\begin{array}{l}\text { Necrosis in } \\
\text { histology } \\
\text { Yes } \\
\text { No }\end{array}$ & $\begin{array}{l}16(34.8 \%) \\
30(65.2 \%)\end{array}$ & $\begin{array}{l}15(55.6 \%) \\
12(44.4 \%)\end{array}$ \\
\hline $\begin{array}{l}\text { Necrosis on } \\
\text { imaging }\end{array}$ & & \\
\hline
\end{tabular}




\begin{tabular}{|c|c|c|}
\hline $\begin{array}{l}\text { Yes } \\
\text { No }\end{array}$ & $\begin{array}{l}4(8.7 \%) \\
42(91.3 \%)\end{array}$ & $\begin{array}{l}5(18.5 \%) \\
22(81.5 \%)\end{array}$ \\
\hline $\begin{array}{l}\text { SN radioactivity } \\
\text { detected with } y \text { - } \\
\text { probe and } \\
\text { camera } \\
\text { Yes } \\
\text { No }\end{array}$ & $\begin{array}{l}40(87.0 \%) \\
6(13.0 \%)\end{array}$ & $\begin{array}{l}5(18 \%) \\
22(81.5 \%)\end{array}$ \\
\hline $\begin{array}{l}\text { Injection } \\
\text { modality } \\
\text { UH } \\
\text { CT }\end{array}$ & $\begin{array}{l}45(97.8 \%) \\
1(2.2 \%)\end{array}$ & $\begin{array}{l}27(100 \%) \\
0\end{array}$ \\
\hline $\begin{array}{l}\text { }{ }^{99 m} \text { Tc dose, } \mathrm{MBq} \\
\text { (median, IQR) }\end{array}$ & $209(187-222)$ & $212(196-218)$ \\
\hline $\begin{array}{l}\text { Number of } \\
\text { injections } \\
1 \text { injection } \\
2 \text { injections } \\
3 \text { injections }\end{array}$ & $\begin{array}{l}27(58.7 \%) \\
18(39.1 \%) \\
1(2.2 \%)\end{array}$ & $\begin{array}{l}18(66.7 \%) \\
9(33.3 \%) \\
0\end{array}$ \\
\hline $\begin{array}{l}\text { Spillage } \\
\text { Yes } \\
\text { No } \\
\text { NA }\end{array}$ & $\begin{array}{l}13(28.3 \%) \\
30(65.2 \%) \\
3(6.5 \%)\end{array}$ & $\begin{array}{l}4(14.8 \%) \\
17(63 \%) \\
6(22.2 \%)\end{array}$ \\
\hline $\begin{array}{l}\text { Depot properly } \\
\text { Injected } \\
\text { Yes } \\
\text { No } \\
\text { NA }\end{array}$ & $\begin{array}{l}30(65.2 \%) \\
2(4.3 \%) \\
14(30.4 \%)\end{array}$ & $\begin{array}{l}14(51.9 \%) \\
1(3.7 \%) \\
12(44.4 \%)\end{array}$ \\
\hline
\end{tabular}




\begin{tabular}{|c|c|c|}
\hline $\begin{array}{l}\text { Anterior depot } \\
\text { Posterior depot } \\
\text { Not anterior nor } \\
\text { posterior }\end{array}$ & $\begin{array}{l}19(41.3 \%) \\
18(39.1 \%) \\
9(19.6 \%)\end{array}$ & $\begin{array}{l}11(40.7 \%) \\
9(33.3 \%) \\
7(25.9 \%)\end{array}$ \\
\hline $\begin{array}{l}\text { Number of } \\
\text { depots } \\
1 \\
2 \\
3 \\
\text { Data not available }\end{array}$ & $\begin{array}{l}26(59.1 \%) \\
17(38.6 \%) \\
0 \\
3(6.5 \%)\end{array}$ & $\begin{array}{l}13(48.1 \%) \\
5(18.5 \%) \\
2(7.4 \%) \\
7(25.9 \%)\end{array}$ \\
\hline $\begin{array}{l}\text { SN Visualization: } \\
\text { On early planar } \\
\text { scintigraphy } \\
\text { On late planar } \\
\text { scintigraphy } \\
\text { On early planar } \\
\text { scintigraphy } \\
\text { combined with } \\
\text { SPECT/CT }\end{array}$ & $\begin{array}{l}5(10.9 \%) \\
9(19.6 \%) \\
32(69.6 \%)\end{array}$ & NA \\
\hline $\begin{array}{l}\text { SN number on } \\
\text { imaging (mean, } \\
\text { IQR, sum) }\end{array}$ & $1.1(0-2,80)$ & NA \\
\hline $\begin{array}{l}\text { SN number } \\
\text { harvested at } \\
\text { surgery (mean, } \\
\text { IQR, sum) }\end{array}$ & $2.1(0-3,155)$ & NA \\
\hline $\begin{array}{l}\text { Non SN number } \\
\text { harvested at } \\
\text { surgery (mean, } \\
\text { IQR, sum) }\end{array}$ & $3.0(0-4,220)$ & NA \\
\hline $\begin{array}{l}\text { Time between } \\
\text { injection and } 1^{\text {st }} \\
\text { imaging in } \\
\text { minutes (median, } \\
\text { IQR) }\end{array}$ & $\begin{array}{l}33 \mathrm{~min}(19 \mathrm{~min}- \\
51 \mathrm{~min})\end{array}$ & $\begin{array}{l}28 \mathrm{~min}(15 \mathrm{~min}-1 \mathrm{~h} \\
5 \mathrm{~min})\end{array}$ \\
\hline
\end{tabular}




\begin{tabular}{|l|l|l|}
\hline $\begin{array}{l}\text { Time between } \\
\text { injection and } \mathbf{2}^{\text {nd }}\end{array}$ & $\begin{array}{l}149 \mathrm{~min}(175 \mathrm{~min}- \\
188 \mathrm{~min})\end{array}$ & $\begin{array}{l}182 \mathrm{~min} \text { (139 } \\
\mathrm{min}-194 \mathrm{~min})\end{array}$ \\
$\begin{array}{l}\text { Imaging in } \\
\text { minutes (median, } \\
\text { IQR) }\end{array}$ & $22.4(4.0-51.6)$ & $6.6(1.5-17.6)$ \\
\hline $\begin{array}{l}\text { Kidney/liver } \\
\text { activity ratio at } \\
\text { early scintigraphy } \\
\text { (median, IQR) }\end{array}$ & $10.7(3.7-26.9)$ & $3.6(1.5-11.4)$ \\
\hline $\begin{array}{l}\text { Kidney/liver } \\
\text { activity ratio at } \\
\text { late scintigraphy } \\
\text { (median, IQR) }\end{array}$ & & \\
\hline
\end{tabular}

Legend: BMI body mass index; IQR interquartile range; SN sentinel node; NA not applicable 


\section{CHAPTER 7}

Sentinel Lymph Node Biopsy in Renal Tumors: Surgical Technique and Safety

Teele Kuusk, Oscar Brouwer, Niels Graafland, Kees Hendricksen, Maarten Donswijk, and Axel Bex

Urology. 2019 Aug;130:186-190. 


\section{ABSTRACT}

OBJECTIVE: To understand uncertainties and knowledge gaps regarding lymphatic drainage in renal tumors, we performed 2 prospective studies to demonstrate regional lymph node (LN) drainage with sentinel lymph node (SN) imaging and biopsy. Here, we report the technique and perioperative safety of retroperitoneal SN dissection with different surgical approaches.

METHODS: Seventy three patients from the 2 trials were included in the analysis. Patients had cT12 NOMO renal tumors $(</=10 \mathrm{~cm})$ and underwent nephrectomy $(46 / 63 \%)$ or partial nephrectomy (27/37\%) with SN dissection after intraoperative detection with a g-probe, and locoregional LND. Twenty-nine of 73 patients had open surgery, 27 of 73 laparoscopic, and 17 of 73 robot-assisted laparoscopic (partial) nephrectomy. Surgery time, intraoperative adverse events (AE) according to CTCAE 5.0, and postoperative AE according to Clavien-Dindo (CD) were retrospectively assessed. RESULTS: There were no grade $\geq 3$ intraoperative CTCAE 5.0 AEs. Postoperative AE rate was $16.4 \%$ of which 7 (9.6\%) were CD grade 1-2 and 5 (6.8\%) were 3a grade complications. There were no statistically significant differences between presence of $A E, C D$ grade, and surgical modality $(P=.27$ and $\mathrm{P}=.13$, respectively). Blood loss was a median of $550 \mathrm{ml}$ (IQR 200-900 $\mathrm{ml}$ ) and 225 (IQR 42-751 ml) for partial nephrectomy (PN) and radical nephrectomy, respectively. Length of the procedure was 170 minutes (IQR 149-184 minutes), 155 minutes (IQR 130-177 minutes) 180 minutes (IQR 162-202 minutes) in open, laparoscopic, and robot-assisted procedures, respectively. CONCLUSION: The addition of retroperitoneal SN dissection combined with locoregional LND during (partial) nephrectomy is surgically safe. Complication rate is low and does not differ between surgical approaches. 


\section{INTRODUCTION}

Decreased morbidity compared to full template lymph node dissection (LND) and locating the first lymphatic landing sites are the key elements of lymph node mapping and biopsy [1]. Sentinel lymph node biopsy (SLNB) has been studied with promising results in different urological cancers with the longest history and indication established in penile cancer [2]. Even though SLNB is experimental in renal cancer, it has shown to identify lymphatic drainage from renal tumours $[3,4]$ and may gain importance for improved staging of high-risk tumors eligible for adjuvant immunotherapy trials as well as for translational studies of early metastasis and immune response. The feasibility of sentinel lymph node detection in renal cancer has previously been published; however the surgical safety of retroperitoneal SLNB has not been reported $[5,6,7]$. Furthermore, there is lacking comprehensive description of the surgical procedure. Our study combined SLNB with locoregional retroperitoneal LND, therefore surgical morbidity could be expected to be the same or even higher than in partial (nephrectomies) with LND alone. The aim of this study was to analyze intra- and postoperative adverse events of SLNB combined with locoregional non-SN LND and describe the procedure in detail.

\section{PATIENTS AND METHODS}

We performed a prospective single center feasibility and phase 2 imaging study of sentinel lymph node (SN) detection and biopsy to investigate the pattern of lymphatic drainage and a rate of LN metastases in renal tumors. The studies were conducted after institutional ethics board approval between 2008 and 2017 (N06SNR and N08SNR; registered under NL26406.031.08 at www.ccmo.nl). All patients signed an informed consent form. Inclusion criteria were cT1-2 renal tumors $\leq 10 \mathrm{~cm}$ of any subtype, clinically and radiologically nonmetastatic disease (cNOMO), age $\geq 18$ years, life expectancy $>3$ months, WHO performance status $0-1$, and no prior systemic therapy. The procedure of SLNB was performed as described earlier[3,7]. Briefly, one day prior to surgery, $225 \mathrm{MBq}$ of $99 \mathrm{mTC}$ nanocolloid (Nanocoll; GE Healthcare, Eindhoven, the Netherlands) in a volume of $0.4 \mathrm{ml}$ was injected into the tumor percutaneously by ultrasound guidance. After 20 minutes and 2-4 hours planar lymphoscintigraphy of the thorax and abdomen was performed, followed by combined SPECT and low dose CT (SymbiaT, Siemens, Erlangen, Germany) of the site of interest. The following day, resection of the primary tumor and the SN was performed. Surgical approach (open, transperitoneal laparoscopic, robotassisted) was decided per case depending on the primary tumor. At surgery, $\mathrm{SN}(\mathrm{s})$ were located by preoperative SPECT/CT images and detected intraoperatively with a g-probe (Neoprobe, Johnson\& Johnson Medical, Hamburg, Germany) in combination with a mobile g-camera (Sentinella, S102,GEM imaging, Valencia, Spain) (Fig. 1,2). After SN excision, the surgical area was scanned using the mobile g-camera to verify complete $\mathrm{SN}$ removal. For ethical reasons, only SNs accessible through the chosen surgical approach were removed. Additionally, non-SNs within the locoregional retroperitoneal LND area were resected to study the false-negative rate. Harvested SNs and non-SNs were measured ex vivo with both g-probe and camera. Surgery time, intraoperative adverse events (AE) according to CTCA 5.0 and blood loss were documented on surgical and anesthesia reports. Postoperative 30-day and the 90-day morbidity and mortality and Aes were 
collected from the patient files. All the procedures were performed by an experienced uro-oncology surgeon (A.B).

\section{INTRAOPERATIVE SURGICAL TECHNIQUE OF SENTINEL LYMPH NODE PROCEDURE}

The renal tumor was removed by open transperitoneal, transperitoneal laparoscopic, and robotassisted laparoscopic (da Vinci S[i] Surgical system [Intuitive Surgical Inc., Sunnyvale, CA, ]) approaches as partial or radical nephrectomy was decided on per case based on individual tumor location and complexity. Independent of the surgical approach, the procedure of SLNB took place after mobilization of the kidney. First, the g-probe wasused to detect the $\mathrm{SN}(\mathrm{s})$ in the anatomical location assessed on SPECT/CT. For the laparoscopic (robotassisted) approach, the laparoscopic probe was inserted through the assistant port or any other of convenience (Fig. 1A, B). In open surgery, in case of activity with a g-probe, we reconfirmed the location of the SNs with a mobile gcamera in vivo (Fig. 1C, D). With the laparoscopic approach, the camera was used ex vivo only. LN which showed any activity with one or the other SN detection methods were harvested and radionuclide activity was reconfirmed with a g-probe and camera ex vivo. Subsequently, excision of non-SNs was performed in accordance with the suggested LND template: in the right side hilar, prepararetrocaval and interaortocaval nodes and from left side hilar, prepara-aortal, and interaortocaval nodes cranially from the crus of diaphragm and distally to the bifurcation of the aorta. Hemostasis and lymph leakage was controlled by bipolar cautery/dissector or metallic clips.

\section{STATISTICAL ANALYSIS}

Statistical analysis was performed using descriptive statistics reporting continues variables with medians and IQRs and categorical variables with proportions. Characteristics of patients and procedures were compared between patients who did and did not develop AE using Fisher's exact, Chi-Square or Mann-Whitney $U$ test. All tests were two-sided and $P$ value $\leq .05$ was considered statistically significant. Data was analyzed using SPSS version 22 (IBM, Chicago, IL).

\section{RESULTS}

Seventy-three patients with a median age of 59 years (IQR 52-65) were included in the final analysis (Table 1), from which 45 patients $(61.6 \%)$ had SN detection either with SPECT/CT or intraoperatively with a g-probe, the remaining patients had locoregional LND. Median size of the tumors was $6 \mathrm{~cm}$ (IQR 4.9-8 cm) and more than half of the cases $43(58.9 \%)$ had high RENAL complexity score. The majority underwent nephrectomy $46(62.8 \%)$ and laparoscopic surgery outweighed the other surgical approaches. Median operating time was 170 minutes (IQR 149-187) and median blood loss was 300 $\mathrm{ml}$ (IQR 100-752). The highest rate of blood loss occurred with open surgery, median $865 \mathrm{ml}$ (IQR 600-1505 ml), laparoscopic 50 (IQR 0-200 ml), and robot-assisted laparoscopic $250 \mathrm{ml}$ (IQR 151-575 $\mathrm{ml}$ ). Length of the procedure was 170 minutes (IQR 149-184 minutes), 155 minutes (IQR 130-177 minutes) 180 minutes (IQR 162-202 minutes) for open, laparoscopic, and robotassisted procedure, respectively. This includes the additional time for SN detection and regional LND which was a median of 30 minutes (IQR 12-52 minutes) and did not statistically differ between the surgical approaches. 
Except blood loss, no intraoperative complications occurred. Blood loss was unrelated to SN and LND. Median follow-up was 52 months (IQR 13-72 months). Postoperative AE rate was $16.4 \%$ (12/73) from which 7 (9.6\%) were Clavien-Dindo grade 1-2 and 5 (6.8\%) were grade 3a complications ( 3 with open surgery and 2 with robotassisted) (Table 2). The majority (10/12) of the complications occurred within 30 days after the operation. Only 1 of these AE was linked to SLNB or LND (lymph fluid collection after open RN). Open partial nephrectomies had the highest number of $A E$, however the approach (open vs laparoscopic vs robotassisted) and technique (partial vs nephrectomy) were not associated with having $C D$ complications or not $P=.13$ and $P=.14$, respectively. Complications were not associated with the number of harvested SNs $(P=.22)$ nor with the number of non-SNs removed during the LND ( $\mathrm{P}=.73)$. Intraoperative detection with SPECT/CT visualization did not differ significantly between surgical approaches $(P=.42)$.

\section{DISCUSSION}

In this study, we explored the surgical safety of retroperitoneal SLNB in renal tumors. We showed that the procedure performed either as open, laparoscopic, or robotassisted approach has no additional intraoperative complications and has acceptable long-term perioperative morbidity. Our single-center results suggest that SLNB in renal tumors has a perioperative AE rate that is comparable or even less than the reported morbidity with LND and nephrectomy.8,9 To our knowledge, there are no prior studies reporting perioperative AEs following a technique of retroperitoneal SLNB in renal tumors using different surgical approaches. Sherif et al studied feasibility of SLNB in renal cancer in 13 patients who underwent nephrectomy but did not report on long-term morbidity nor details of the SLNB procedure. 5 In renal cancer locoregional LND is challenging due to approximation of large blood vessels. Despite that, data on LND in renal cancer suggest that there is no significant additional impact on complications compared to no LND in patients who underwent a nephrectomy ( $26 \% \mathrm{vs}$ $22 \%$, respectively) whereas lymph fluid drainage was reported in $2.4 \%$ and $3.9 \%$, respectively. 8 Furthermore, a previously published meta-analysis reported a perioperative complication rate with nephrectomy and LND in $17 \%-26 \%, 9$ whereas our AE rate was $16.4 \%$ without any intraoperative complication directly linked to SLNB, demonstrating safety and minimal morbidity of the procedure. Regarding intraoperative blood loss and surgery time, there are great variations in different publications. In retrospective series mean blood loss has been 856-1301 ml for open nephrectomy and LND with a mean surgery time of $178 \mathrm{~min}, 9,10$ whereas in the laparoscopic setting, $150 \mathrm{ml}$ and 217 minutes were reported, respectively.11 In our series, mean surgery time was 173 minutes, with mean blood loss of $1286 \mathrm{ml}$, which was 166 minutes and $142 \mathrm{ml}$ for the laparoscopic approach, respectively. Generally, SN procedure with LND is 10-50 minutes longer than (partial) nephrectomy with LND alone. The duration of robot-assisted laparoscopic operations was the longest; however this was predominantly due to the majority of patients having a partial rather than total nephrectomy. In addition, the study period coincided with the beginning of learning curve for robot assisted laparoscopic partial nephrectomy (RALPN) in our institute. One of the main reasons for SN mapping and biopsy is to detect the first draining lymph nodes and to reduce the morbidity of extended LND without diminishing oncological outcome. Owing to a lack of evidence that LND provides oncological advantage in renal cancer, 8 LND is not part of a standard nephrectomy. However, abandoning LND in renal cancer entirely is controversial. Lymph node metastases are the third most common metastatic sites 12 and are associated with an extremely poor outcome. 9,13 
Finding better therapeutic options for these patients is critical and multiple adjuvant studies are ongoing. For this reason, evaluating the presence of occult lymph node metastatic disease in highrisk renal cancer is gaining importance for staging purposes as lymphonodular involvement is part of the inclusion criteria in novel adjuvant immunotherapy and combination therapy trials. Furthermore, it has been shown that there is a subgroup of patients who survive longer when occult lymph node metastases are removed early.9,13 Instead of performing extended LND which detects only a small number of occult lymph nodes (4\%-8\%), it may be convenient to use SLNB instead. Nevertheless, we do not have a comparative study between SLNB and extended LND in renal cancer. SLNB could theoretically save procedural time and would also guide the surgeon to the first lymphatic landing sites for performing SPECT/CT guided selected LND for staging rather than removing all retroperitoneal locoregional lymph nodes around the aorta and caval vein. Furthermore, due to aberrant lymphatic drainage in renal tumors (35\%), SNLB3 can be used for mapping and directing LND. The new technique adaptation and performance is feasible in expert hands and SLNB could be used in trials where staging is needed.14 For further lymphatic drainage trials in high risk renal cancer patients, it is of importance to demonstrate that perioperative $A E$ and long term morbidity following SLNB is not exceeding renal surgery without LND. Our study is not without limitation. It is retrospective and lacks a group for comparison. Also the low number of patients per surgical approach limits the interpretation of the findings.

\section{CONCLUSION}

The results suggest long-term safety and low morbidity of retroperitoneal SLNB for renal tumors patients who undergo (partial) nephrectomy and LND.

\section{ETHICAL APPROVAL}

All procedures performed in studies involving human participants were in accordance with the ethical standards of the institutional and/or national research committee and with the 1964 Helsinki Declaration and its later amendments or comparable ethical standards.

\section{INFORMED CONSENT}

Informed consent was obtained from all individual participants included in the study.

Conflicts of interest: The authors declare that they have no conflict of interest.

Acknowledgments We are grateful for Antoni Van Leeuwenhoek Netherlands Cancer Institute team members for their contribution to the study. 


\section{References}

1. INTERNATIONAL ATOMIC ENERGY AGENCY. Guided Intraoperative Scintigraphic Tumour Targeting (GOSTT), IAEA Human Health Series No. 29. Vienna: IAEA; 2014.

2. Jakobsen JK. Sentinel node biopsy in uro-oncology: A history of the development of a promising concept. Urol Oncol. 2015;33: 486-493.

3. Kuusk T, De Bruijn R, Brouwer RO. Lymphatic drainage from renal tumors in vivo: a prospective sentinel node study using SPECT/CT imaging. J Urol. 2018;199:1426-1432.

4. Kuusk T, De Bruijn R, Brouwer OR. Outcome of sentinel lymph node biopsy in patients with clinically non-metastatic renal cell carcinoma. Scand J Urol. 2018;11:1-8.

5. Sherif AM, Eriksson E, Th€orn M. Sentinel node detection in renal cell carcinoma. A feasibility study for detection of tumour-draining lymph nodes. BJU Int. 2012;109:1134-1139.

6. Bex A, Vermeeren L, de Windt G, Prevoo W, Horenblas S, Olmos RA. Feasibility of sentinel node detection in renal cell carcinoma: a pilot study. Eur J Nucl Med Mol Imaging. 2010;37: 1117-1123.

7. Bex A, Vermeeren L, Meinhardt W, Prevoo W, Horenblas S, Valdes Olmos RA. Intraoperative sentinel node identification and sampling in clinically node-negative renal cell carcinoma: initial experience in 20 patients. World J Urol. 2011;29:793-799.

8. Blom JHM, Van Poppel H, Marechal JM, et al. Radical nephrectomy with and without lymph-node dissection: final results of European Organization for Research and Treatment of Cancer (EORTC) randomized phase 3 trial 3088. Eur Urol. 2009;55: 28-34.

9. Bhindi B, Wallis CJD, Boorjian SA, et al. The role of lymph node dissection in the management of renal cell carcinoma: a systematic review and meta-analysis. BJU Int. 2018;121: 684-698.

10. Pantuck AJ, Zisman A, Dorey F, et al. Renal cell carcinoma with retroperitoneal lymph nodes: role of lymph node dissection. J Urol. 2003;169:2076-2083.

11. Chapman TN, Sharma S, Zhang S, Wong MK, Kim HL. Laparoscopic lymph node dissection in clinically node negative patients undergoing laparoscopic nephrectomy for renal carcinoma. Urology.

$2008 ; 71: 287-291$.

12. Chandrasekar T, Klaassen Z, Goldberg H, et al. Metastatic renal cell carcinoma: patterns and predictors of metastases-a contemporary population-based series. Urol Oncol. 2017;35:661.e7661.e14.

13. Babaian KN, Kim DY, Kenney PA. Preoperative predictors of pathological lymph node metastasis in patients with renal cell carcinoma undergoing retroperitoneal lymph node dissection. J Urol.

2015;193:1101-1107.

14. Cramer JD, Sridharan S, Ferris RL, Duvvuri U, Samant S. Sentinel lymph node biopsy versus elective neck dissection for stage I to II oral cavity cancer. Laryngoscope. 2019 Jan;129(1):162-169. 
Figure 1.
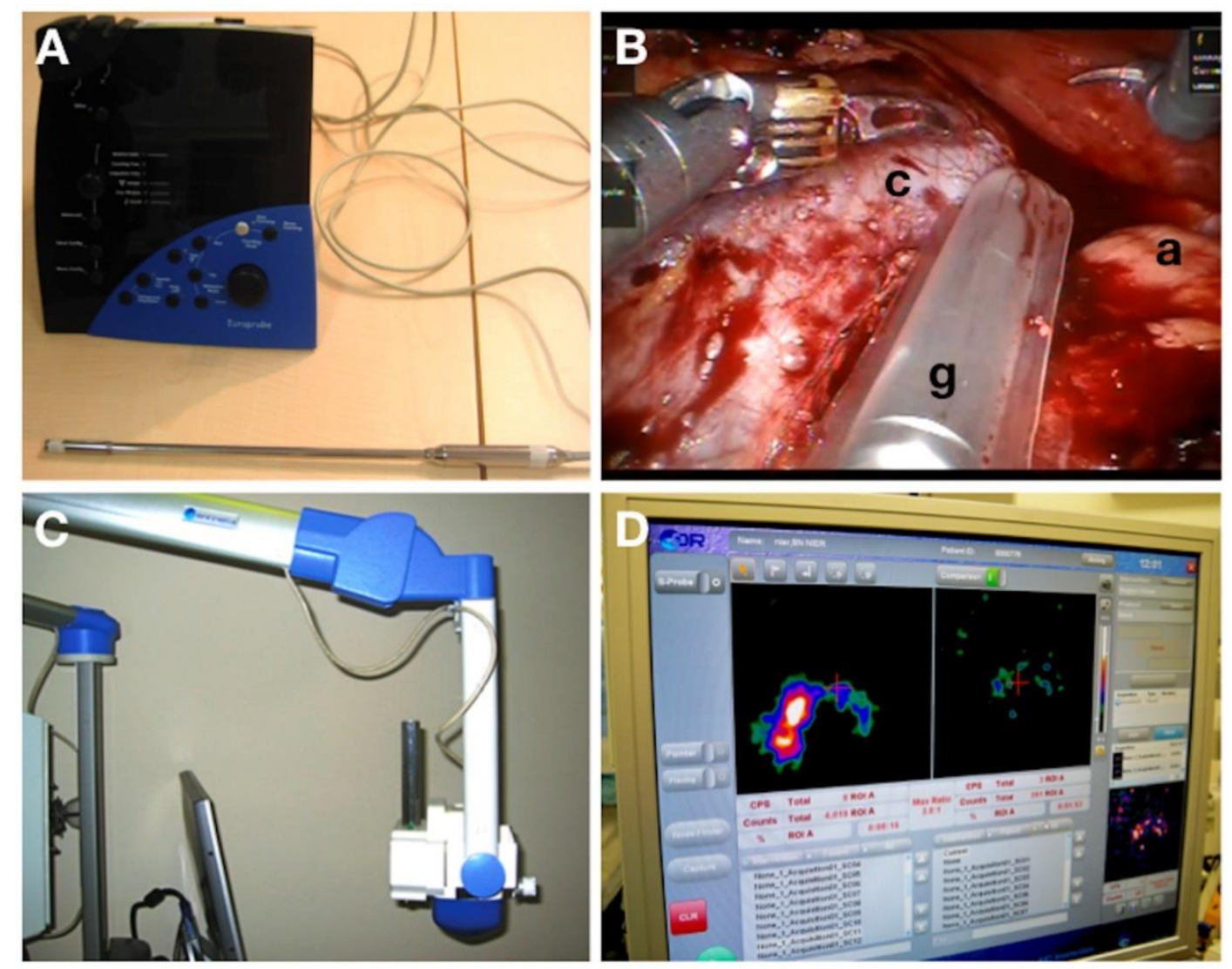

Legend: A. $\gamma$-probe tool B. $\gamma$-probe detecting interaortocaval SN intraoperatively c (caval vein) a (aorta) g ( $\gamma$-probe) $\mathrm{C}$. Sentinella mobile $\gamma$-camera $\mathrm{D}$. Sentinella mobile $\gamma$-camera monitor image showing detection of SN

Figure 2

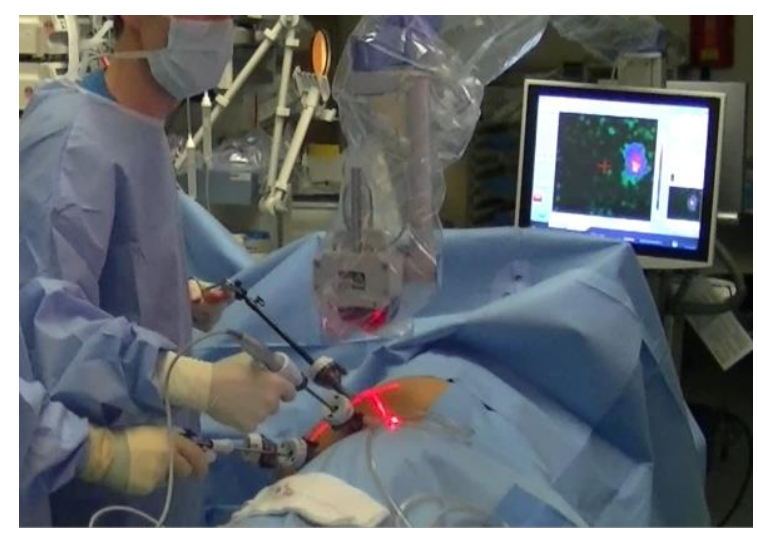

Sentinel lymph node detection with a $y$-camera during laparoscopic surgical procedure. 
Table 1. Patient and tumor characteristics

\begin{tabular}{|c|c|}
\hline Number of patients & 73 \\
\hline Age (median, IQR) & $59(52-65)$ \\
\hline BMI (median, IQR) & $26.4(24.1-30.9)$ \\
\hline Tumour size in cm (median, IQR) & $6(4.9-8)$ \\
\hline \multicolumn{2}{|l|}{ pT stage } \\
\hline T1a & $11(15.1 \%)$ \\
\hline $\mathrm{T} 1 \mathrm{~b}$ & $43(58.9 \%)$ \\
\hline $\mathrm{T} 2 \mathrm{a}$ & $8(11.0 \%)$ \\
\hline $\mathrm{T} 2 \mathrm{~b}$ & $3(4.1 \%)$ \\
\hline T3а & $8(11.0 \%)$ \\
\hline \multicolumn{2}{|l|}{ pN stage } \\
\hline NO & $65(89.0 \%)$ \\
\hline N1 & $2(2.7 \%)$ \\
\hline $\mathrm{Nx}$ & $6(8.2 \%)$ \\
\hline Right side & $36(49.3 \%)$ \\
\hline Left side & $37(50.7 \%)$ \\
\hline Upper pole & $15(20.5 \%)$ \\
\hline Intermedial pole & $31(42.5 \%)$ \\
\hline Lower pole & $27(37.0 \%)$ \\
\hline \multicolumn{2}{|l|}{ Histology } \\
\hline Clear cell RCC & $52(71.2 \%)$ \\
\hline Papillary type 1 RCC & $7(9.6 \%)$ \\
\hline Papillary type 2 RCC & $4(5.5 \%)$ \\
\hline Chromophobe RCC & $5(6.8 \%)$ \\
\hline Oncocytoma & $3(4.1 \%)$ \\
\hline Solitary fibrous tumor & $1(1.4 \%)$ \\
\hline Leibovich score in ccRCC & \\
\hline
\end{tabular}




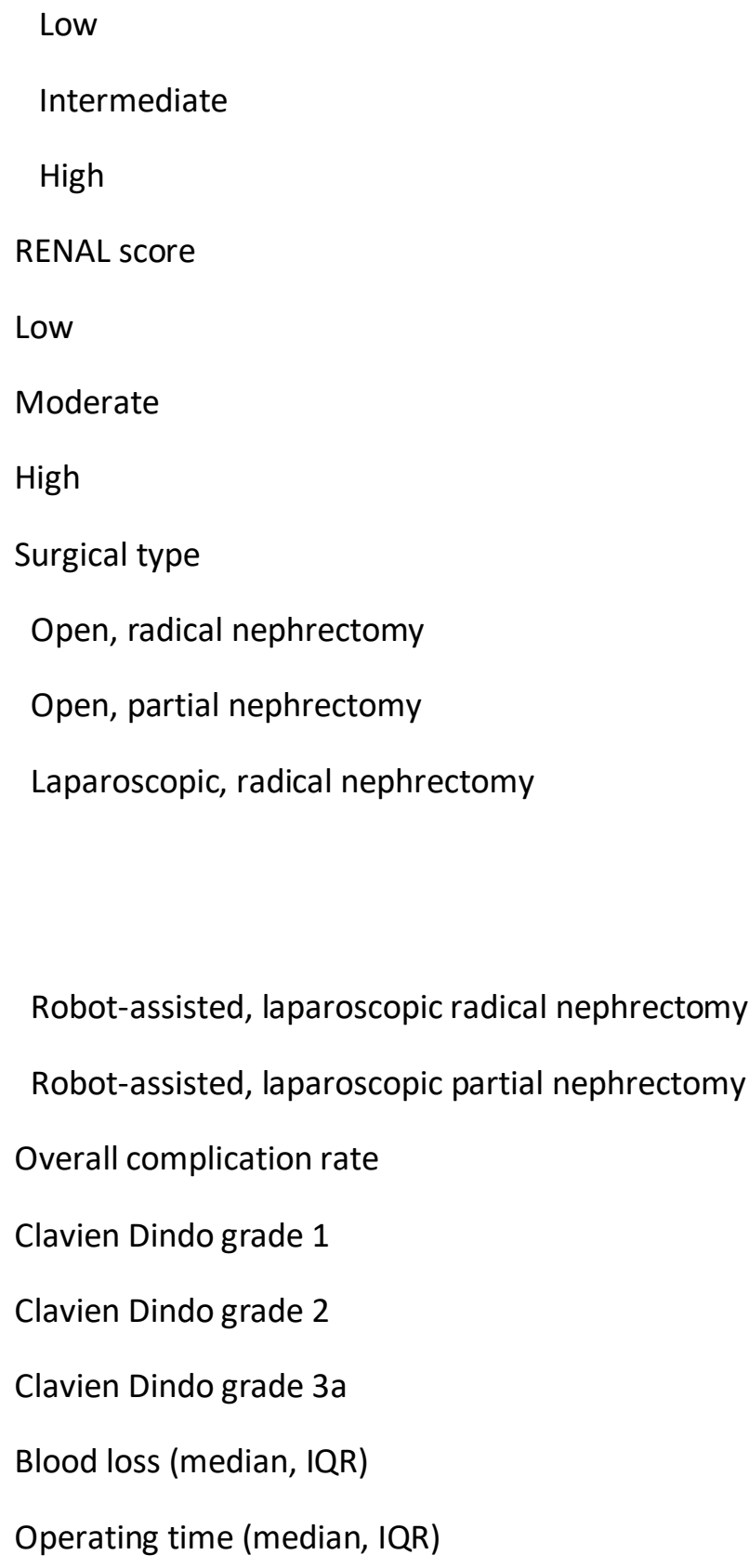

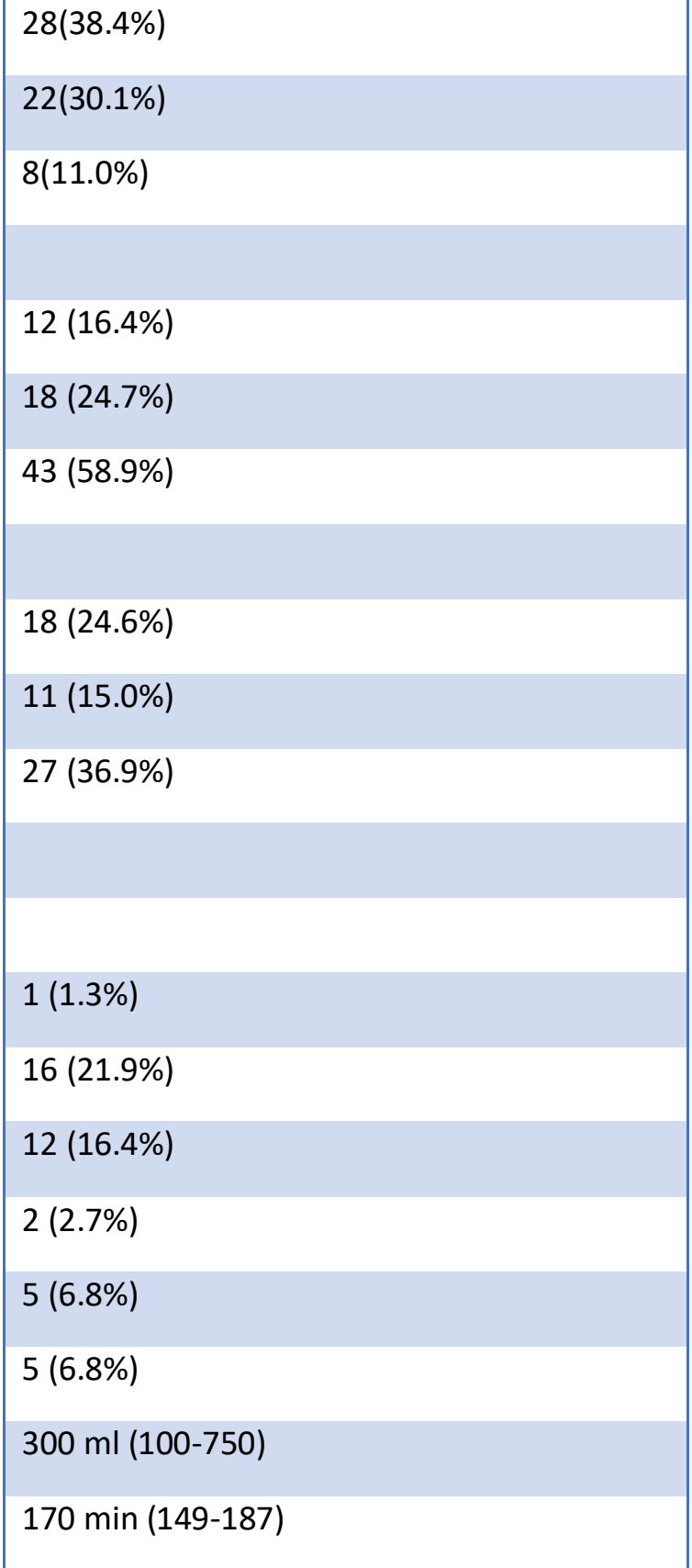

Legend: IQR interquartile range 
Table 2. Patients with complications

\begin{tabular}{|c|c|c|c|c|c|c|c|c|c|c|}
\hline $\begin{array}{l}\text { Patient } \\
\mathrm{nr}\end{array}$ & $\begin{array}{l}C D \\
\text { grade }\end{array}$ & Age & Gender & $\begin{array}{l}\mathrm{Si} \\
\mathrm{d} \\
\mathrm{e}\end{array}$ & $\begin{array}{l}\text { Tumo } \\
\text { ur } \\
(\mathrm{cm})\end{array}$ & $\begin{array}{l}\text { pT } \\
\text { stage }\end{array}$ & $\begin{array}{l}\text { RENAL } \\
\text { score }\end{array}$ & Surgery & Complication & Treatment \\
\hline
\end{tabular}

\begin{tabular}{|c|c|c|c|c|c|c|c|c|c|c|c|}
\hline 1 & 2 & 63 & Male & $\mathrm{R}$ & 4.0 & $1 b$ & Low & RAPN & DVT & Anticoagulants & 39 \\
\hline 2 & $3 a$ & 63 & Male & $\mathrm{L}$ & 5.6 & $1 b$ & High & RAPN & Bladder clot retention & $\begin{array}{l}\text { Transurethral } \\
\text { evacuation }\end{array}$ & 26 \\
\hline 3 & $3 a$ & 39 & Female & $\mathrm{L}$ & 5.7 & $1 b$ & Mod & RAPN & AVF & Endovascular Coiling & 13 \\
\hline 4 & 2 & 47 & Male & $\mathrm{R}$ & 3.5 & $1 a$ & Mod & RAPN & Pneumonia & $A B$ & 3 \\
\hline 5 & 2 & 74 & Female & $\mathrm{L}$ & 7.0 & $1 b$ & High & Lap RN & $\mathrm{AF}$ & Medication & 5 \\
\hline 6 & 1 & 56 & Male & $\mathrm{R}$ & 6.0 & $3 a$ & Mod & Open RN & Wound infection & Topical dressings & 7 \\
\hline 7 & $3 a$ & 72 & Female & $\mathrm{R}$ & 4.8 & $1 b$ & High & Open RN & $\begin{array}{l}\text { Subcutaneous lymph } \\
\text { fluid collection }\end{array}$ & Drainage & 3 \\
\hline 8 & 1 & 55 & Female & $\mathrm{R}$ & 5.0 & $1 b$ & High & Open PN & Wound infection & Topical dressings & 5 \\
\hline 9 & $3 a$ & 59 & Female & $\mathrm{L}$ & 5.5 & $1 b$ & High & Open RN & Wound hernia & Surgical repair & 60 \\
\hline 10 & 3 & 54 & Female & $\mathrm{L}$ & 3.5 & $1 a$ & Low & Open PN & Urine leakage & Double-J stent & 5 \\
\hline 11 & 2 & 67 & Male & $\mathrm{R}$ & 6.5 & $1 b$ & High & Open RN & Pneumonia & $A B$ & 4 \\
\hline 12 & 2 & 77 & Female & $\mathrm{R}$ & 8.0 & $3 a$ & High & Open RN & Pneumonia & $A B$ & 6 \\
\hline
\end{tabular}


PO post-operative; DVT deep vein thrombosis; R right; L left; Mod Moderate; AVF arteriovenous fistula; $A F$ artrial fibrillation; AB antibiotics; RN radical nephrectomy; PN partial nephrectomy; Lap laparoscopic; RALPN robotic-assisted laparoscopic partial nephrectomy 


\section{CHAPTER 8}

\section{Summary and conclusions}

Lymph node status is a major prognostic factor in renal cancer after radical or partial nephrectomy. However, currently most accurate diagnostic tool for lymph node invasion, lymph node dissection (LND), does not routinely belong to the surgical management of renal cancer. This is due to its lack of evidence of therapeutic efficacy. Cross-sectional imaging to detect occult lymph node metastases in patients with clinically node-negative disease (cNO) cannot replace LND as it has a low diagnostic sensitivity and specificity. Yet, new promising therapeutic options with immunotherapy have reintroduced interest in LND for prognostic purposes. As a consequence, knowledge gaps in lymphatic drainage in renal tumours have encouraged the exploration of sentinel lymph node imaging with SPECT/CT and sentinel lymph node biopsy, which combines anatomical information of the first draining lymphatic nodes with histopathologic examination.

Chapter 1 gives insight into a changing treatment paradigm of advanced and metastatic renal cell carcinoma. New immune checkpoint inhibitor therapy has revolutionized the treatment of systemic disease and impressive improvements in outcome have been reported. Now, combination therapies with antiangiogenic and various immunotherapies are being researched actively as adjuvant or neoadjuvant therapies in patients with high-risk of recurrence, including those with resected lymph node metastases. The first adjuvant trial results are awaited in a few years time and if these will show positive outcome in survival then LND in renal cancer will most likely be re-evaluated for prognosis assessment. Evaluation of lymphatic drainage patterns may be of importance to design more accurate templates.

Chapter $\mathbf{2}$ assessed anatomical lymphatic drainage in renal tumours. Results were from a phase II prospective single arm study using imaging with lymphoscitigraphy and SPECT/CT in vivo. It revealed high individual variability in the location of the sentinel lymph nodes. The majority of the patients (26 of 40 [65\%]) had drainage exclusively into the retroperitoneal local lymph node basins, which are commonly included in extended LND templates, however 14 (35\%) of the patients had additional draining lymph nodes outside the retroperitoneum involving also supradiaphragmatic lymph nodes (8[20\%]). The tumours on the right side drained predominantly into interaortocaval and retrocaval sentinel lymph nodes and on the left side the drainage was mainly into paraaortic sentinel nodes. Simultaneous drainage on the right side included interaortocaval, retrocaval, left preaortic or paraaortic and left supraclavicular lymph nodes, whereas drainage to the paracaval, precaval and hilar region was rare. On the left side simultaneous drainage was into hilar, mediastinal, left supraclavicular, retrocrural, left common iliac, renal fossa and interaortocaval sentinel nodes and direct hilar drainage was also rare.

Chapter 3 evaluated the oncological outcome of sentinel lymph node biopsy with LND in renal cancer. We found that the rate of occult lymph node metastases is very low (2 patients out of 66, $3 \%)$. However, these patients had excellent disease-free survival (DFS) of 57 and 72 months with a median follow-up of 57 months (IQR 18-72 months). Recurrence of the disease was detected in 10 
patients (15\% [95\% Cl 7-26\%]), whereas 4 (6\% [95\% Cl 2.3-14.5\%]) died of the disease. There was association with metachronous metastases and tumour size, pT stage, Leibovich score and interaortocaval sentinel lymph node location. Five out of 6 patients with at least one interaortocaval lymph node drainage on imaging developed thoracic metastases which would support the theory of interaortocaval direct drainage through the thoracic duct into the lungs without drainage into the mediastial lymph nodes in between. Long disease free survival should be interpreted cautiously given the low number of patients.

Chapter 4 reported on a patient with a cT1bcNOcMO renal tumour who was enrolled into the sentinel lymph node prospective trial and treated with laparoscopic radical nephrectomy and sentinel lymph node biopsy with completion LND. After pathological examination the stage was upgraded to pT1bpN1 papillary type II RCC. Two sentinel nodes revealed microscopic metastatic lymph nodes, however, the patient remained disease free after 63 months of follow-up. This case demonstrates that some patients with clinically occult lymph nodes and single site lymph node metastases removal may have long survival. Nevertheless, identification of these patients and lymph nodes on routine pre-operative cross sectional imaging is challenging whereas sentinel lymph node imaging and biopsy could guide in determining the first draining lymphatic sites.

Chapter 5 evaluated the topographic distribution of early single-or oligometastatic lymph nodes in renal cancer and aimed to compare whether these sites correspond to the anatomical sites of sentinel lymph nodes on imaging. We found that on the right side single- or oligometastases are predominantly located in the hilar, paracaval, retrocaval and interaortocaval areas and on the left side in the paraaortal region. These areas follow the pattern observed in the prospective sentinel lymph node studies. However, the study is limited by its retrospective nature and absence of a standardized LND template.

Chapter 6 analyzed reasons for the relatively high rate of non-visualization with scintigraphy and SPECT/CT in renal tumours. Visualization rate on imaging was $63 \%$ and the only factor which was associated with non-visualization was older age. Regarding the technique, the majority of sentinel lymph nodes were visualized 2-4 $\mathrm{h}$ after tracer injection. Both the trunk as well as thoracic area should be imaged with planar scintigraphy in combination with SPECT /CT because $20 \%$ patients had the first draining lymph nodes in the supradiaphragmatic area. The dosage of $225 \mathrm{MBq} 99 \mathrm{mTc}$, according to depot activity, was optimal. Due to the limitations in our study which include post hoc analysis, a low number of patients as well as mainly low risk patients with low incidence of lymph node metastatic disease, the cause of non-visualization remains uncertain.

Chapter $\mathbf{7}$ investigated the morbidity of sentinel lymph node biopsy combined with lymph node dissection and (partial) nephrectomy. Overall, the postoperative adverse event rate was $16.4 \%$, of which Clavien Dindo grade 1-2 and 3a represented 9.6\% and 6.8\% respectively. There was no association between complications and applied surgical modality. Overall, the sentinel lymph node procedure in renal cancer is safe in open as well as in minimally-invasive surgery. However the analysis is limited by the absence of a comparative group and also by its post hoc nature. 


\section{Future outlook}

Sentinel node biopsy in clinical application and other Imaging techniques

In the era of immuotherapy prognostication techniques and validated risk models in renal cancer are more frequently applied and lymph node metastasis is an important prognostic factor. Commonly used tools to predict and evaluate lymph node invasion are nomograms, LND or imaging techniques. Risk scores predicting lymph node invasion are hypothetically very valuable tools, however, in renal cancer the low rate of occult lymph node positive disease is the main obstacle in using these in practice [1]. All available nomograms to predict pathological lymph node metastases (pN1) increase in accuracy only when lymph nodes are already enlarged on imaging (cN1). Therefore, if proper prognosis assessment is warranted, LND is still the gold standard and the most accurate method for detecting lymph node invasion. However, to avoid LND morbidity, less invasive methods would be preferred. Sentinel node biopsy is an experimental procedure and clinical application would require further investigation in patients with high-risk renal cell carcinoma and clinically node-negative disease on imaging to determine the potentially higher rate of occult lymph node metastases in this setting. However, the dynamic studies of first landing sites have given us an understanding of the extent of lymphatic drainage with supradiaphragmatic drainage along the thoracic duct which may help to explain why LND for therapeutic use has hitherto been without a proven survival benefit. Nevertheless, the majority of patients have sentinel nodes located in the lymph nodes following the renal arteries and aorta on both sides which could be exploited for prognostic sentinel node biopsy studies in high-risk renal cancer. This may yield data on the frequency of occult lymph node metastatic renal cancer in high-risk disease which, in case of a meaningful metastasis rate, may allow to investigate the role of sentinel node resection as a therapeutic procedure in further prospective trials.

Sentinel node biopsy, which is still an invasive procedure, may have to compete as an experimental technique with emerging non-invasive imaging modalities to detect lymph node involvement for staging and prognosis assessment. Conventional CT detection rate for lymph node metastases is less than $60 \%$ but it can be increased when combined with [89Zr]Zirconium-Desferrioxamine (DFO)girentuximab-PET/CT and [18F] Fluoro-desoxy-glucose FDG-PET/CT imaging [2]. Overall, in a study, [89Zr]Zr-DFO-girentuximab-PET/CT raised the metastatic renal carcinoma lesion detection rate from $56 \%$ to $91 \%$, however, regarding lymph note metastasis detection [18F]FDG-PET/CT had a higher detection rate than [89Zr]Zr-DFO-girentuximab-PET/CT, $85 \%$ and $>90 \%$, respectively. In addition, [89Zr]Zr-DFO-girentuximab-PET/CT can only be used in clear-cell renal cell carcinoma as girentuximab is a monoclonal antibody against carboanhydrase- 9 (CAIX) expressed on clear-cell renal cell carcinoma. Nevertheless, in view of the [18F]FDG-PET/CT false positive detection rate in reactive lymph nodes, [89Zr]Zr-DFO-girentuximab-PET/CT is preferable [2]. The limitation of this study is lack of histologic diagnosis of lymph node metastases. Girentuximab has shown also promising results in a prospective feasibility study in follow up after cryoablation [3]. To conclude, [89Zr]Zr-DFOgirentuximab-PET/CT is a promising combination of anatomical and functional imaging which could hypothetically replace LND for prognosis assessment in the future, however it requires further studies with a larger cohort and evaluation of histology in high risk clear-cell renal cell carcinoma patients. Currently, [89Zr]Zr-DFO-girentuximab-PET/CT is experimental and being investigated in a confirmatory International trial [NCT03849118]. 
Sentinel node biopsy and its role in translational studies

A group from Scandinavia investigated detection of single tumour cells in sentinel nodes from renal cancer which on histological examination did not contain visible metastatic deposits at light microscopy [4]. They demonstrated with staining for the intracellular marker cytokeratin 18 (CK18) together with the surface markers carbonic anhydrase 9 (CAIX) and Cadherin 6 in flow cytometry analysis that almost all sentinel lymph nodes contained single tumour cells at relatively high volumes compared to conventional pathology [4]. However, this observation should be interpreted with caution because only 15 sentinel lymph nodes were evaluated and they used longitudinal cutting of the lymph nodes instead of scraping in their methods which could have had an impact on detecting the micrometastases in one half of the lymph node with immunohistochemistry but not in the other half which was examined with H\&E [4]. Nevertheless, this suggests potential for translational research projects involving sentinel lymph nodes. Knowing that renal cancer is heterogenous and its behaviour unpredictable, single tumour cells sorted by flow cytometry could be sequenced to compare them with the genomic properties of the primary tumour to investigate and understand a potential predeliction for the lymphatic route. In addition, it is unknown which immunogenic changes take place in the lymph nodes receiving first lymphatic drainage from the primary tumour and its microenvironment.

Sentinel lymph node biopsy has not been studied previously in renal cancer, whereas it has been implemented in the management of other genitourinary malignancies $[5,6,7]$. Next to the importance of the sentinel node biopsy for staging and therapeutic decision-making, the sentinel node has been recognized as the lymph node in which priming of the immune reaction takes place. There are detailed reports from investigations performed in and ex vivo in immunogenic malignancy such as melanoma [8]. Sentinel lymph nodes, being the first nodes to receive lymph from a primary tumour and the preferential site of initial tumour metastases, are intensively exposed to the bioactive products of tumour cells and other associated cells. This makes them ideal for studies of the factors that determine selective tissue susceptibility to metastases. In melanoma local administration of Granulocyte-macrophage colony-stimulating factor (GM-CSF) and CpG-B oligonucleotides at the primary melanoma excision site leads to full-range activation of conventional dendritic cells (DCs) ( $C D C$ ) and plasmacytoid DCs ( $p D C$ ) subsets, both in sentinel nodes and in blood. The cross-presenting capacity of sentinel node DCs suggests that their recruitment may contribute to protection against metastatic spread through increased cytotoxic T lymphocyte (CTL) induction against melanoma-derived antigens [8].

Renal cell carcinoma shares many immunologic features with melanoma. Investigation of the immune response in the sentinel node is necessary to understand whether similar mechanisms apply for renal cell carcinoma. In analogy to melanoma, this may open new therapeutic concepts in the future.

Currently, we are planning to evolve our prospective sentinel lymph node biopsy study to characterize the immunological signature of tumor-draining sentinel nodes which could give a unique insight into the interaction of the components of the immune system and malignant cells. The main objective of the study is to describe the characteristics of tumour-associated lyphocytes involved in anti-tumor responses in the primary renal tumor, the tumor draining sentinel node and any regional non-tumor draining but surgically sampled lymph nodes. This involves exploratory comparison of the primary renal tumor, sentinel nodes and regional non-sentinel nodes, by immunohistochemistry and 
RNA-sequencing and correlation of potential findings with clinical outcome (recurrence- free survival, cancer-specific survival or overall survival).

\section{References}

1. Bhindi B, Wallis CJD, Boorjian SA, Thompson RH, Farrell A, Kim, et al. The role of lymph node dissection in the management of renal cell carcinoma: a systematic review and meta- analysis. BJU Int. 2018 May;121(5):684-698.

2. Verhoeff SR, van Es SC, Boon E, van Helden E, Angus L, Elias SG, et al. Lesion detection by [89Zr]ZrDFO-girentuximab and [18F]FDG-PET/CT in patients with newly diagnosed metastatic renal cell carcinoma. Eur J Nucl Med Mol Imaging. 2019;46(9):1931-1939.

3. van Oostenbrugge TJ, Langenhuijsen JF, Oosterwijk E, Boerman OC, Jenniskens SF, Oyen WJG, et al. Follow-up imaging after cryoablation of clear cell renal cell carcinoma is feasible using single photon emission computed tomography with 111In-girentuximab. Eur J Nucl Med Mol Imaging. 2019 Nov 25. doi: 10.1007/s00259-019-04613-z.

4. Hartana CA, Kinn J, Rosenblatt R, Anania S, Alamdari F, Glise H, et al. Detection of micrometastases by flow cytometry in sentinel lymph nodes from patients with renal tumours. $\mathrm{Br} J$ Cancer. 2016:11;115(8):957-966.

5. Sadeghi R, Gholami H, Zakavi SR, Kakhki VR, Tabasi KT, Horenblas S. Accuracy of sentinel lymph node biopsy for inguinal lymph node staging of penile squamous cell carcinoma: systematic review and meta-analysis of the literature. J Urol. 2012;187(1):25-31.

6. Zarifmahmoudi L, Ghorbani H, Sadri K, Tavakkoli M, Keshvari M, Salehi M, et al. Sentinel Node Biopsy in Urothelial Carcinoma of the Bladder: Systematic Review and Meta-Analysis. Urol Int. 2019;103(4):373-382.

7. Wit EMK, Acar C, Grivas N, Yuan C, Horenblas S, Liedberg F, et al. Sentinel Node Procedure in Prostate Cancer: A Systematic Review to Assess Diagnostic Accuracy. Eur Urol. 2017;71(4):596-605.

8. Sluijter BJ, van den Hout MF, Koster BD, van Leeuwen PA, Schneiders FL, van de Ven R, et al. Arming the Melanoma Sentinel Lymph Node through Local Administration of CpG-B and GM-CSF: Recruitment and Activation of BDCA3/CD141(+) Dendritic Cells and Enhanced Cross-Presentation. Cancer Immunol Res. 2015;3(5):495-505. 


\section{Samenvatting}

$\mathrm{Na}$ een radicale of partiële nefrectomie vanwege nierkanker is de lymfeklier status één van de belangrijkste prognostische factoren. Het meest nauwkeurige diagnostische middel dat we hebben om lymfeklier invasie aan te tonen, de lymfeklier dissectie (LND), wordt echter niet standaard uitgevoerd bij een nefrectomie. Dit is te wijten aan het gebrek aan bewijs voor therapeutische werkzaamheid. De lage sensiviteit en specificiteit van conventionele beeldvorming in het opsporen van occulte LN's is ook niet bemoedigend. Nieuwe veelbelovende behandelingen met immunotherapie hebben tot een nieuwe interesse in LND geleid vanwege prognostische redenen. De kenniskloof op het gebied van lymfedrainage van niertumoren heeft de exploratie van schildwachtklier beeldvorming met SPECT/CT en schildwachtklier biopsie aangemoedigd. Hierbij wordt de anatomische informatie van de eerste drainerende lymfeklieren gecombineert met histopathologisch onderzoek.

Hoofdstuk 1 geeft inzicht in een veranderend veld in behandelparadigma's van gemetastaseerd niercelcarcinoom (mRCC). Nieuwe immunotherapie heeft de uitkomst van behandelingen volledig veranderd en combinatietherapieën met anti-angiogene en verschillende immunotherapieën in adjuvante en neo-adjuvante setting worden actief onderzocht. De eerste proefresultaten in adjuvante setting worden verwacht in een paar jaar tijd. Wanneer deze studies overlevingswinst laaten zien, dan zal de waarde van LND bij nierkanker opnieuw worden herzien. Evaluatie van lymfedrainage patronen kan van belang zijn bij het bepalen van meer nauwkeurige templates.

Hoofdstuk 2 beoordeelde anatomische lymfedrainage van niertumoren. Er wordt een fase II prospectieve single-arm studie beschreven waarin anatomische lymfedrainage in niertumoren werd geevalueerd met behulp van beeldvorming met lymfoscintigrafie en SPECT/CT in vivo. Er kwam een hoge individuele variabiliteit in eerste drainerende lymfeklieren naar voren. De meerderheid van patiënten (26 van de $40(65 \%)$ ) had uitsluitend drainage in het retroperitoneale lokale lymfeklierstroomgebied, dat gewoonlijk in een uitgebreid LND-gebied wordt opgenomen. Daarentegen hadden 14 patiënten (35\%) additioneel drainerende lymfeklieren buiten het retroperitoneum, inclusief supradiafragmatische lymfeklieren (8 (20\%)). De tumoren aan de rechterkant draineerden voornamelijk in inter-aortocavale en retrocavale schildwachtklieren en aan de linker kant was de drainage voornamelijk in para-aortale schildwachtklieren. Gelijktijdige drainage van de rechterkant omvatte inter-aortocavale, retrocavale, linker pre-aortale of para-aortale en linker supraclaviculaire lymfeklieren, terwijl drainage naar para-caval, pre-caval en de hilusregio zeldzaam was. Aan de linker kant was gelijktijdige drainage naar de hilaire, mediastinale, linker supraclaviculaire, retrocrurale, links iliacale, para-renale en interaortocavale schildwachtklieren en directe drainage naar de hilus was ook zeldzaam. 
Hoofdstuk 3 evalueerde de oncologische uitkomst van een schildwachtklier bioptie met LND bij nierkanker. Het percentage van occulte lymfeklier metastasen bleek zeer laag. (2 van de 66 patiënten (3\%)). Deze patiënten hadden echter een lange ziektevrije overleving (DFS) van 57 en 72 maanden met een mediane follow-up van 57 maanden (IQR 18-72 maanden). Ziekte-recidief werd gevonden in 10 patiënten (15\% [95\% Cl 7-26\%]), terwijl 4 (6\% [95\% Cl 2.3-14,5\%]) stierf aan de ziekte. Er was associatie met metachrone metastasen en tumorgrootte, pT stadium, Leibovich Score en interaortocavale schildwachtklierlocatie. Vijf van de 6 patiënten met ten minste één interaortocavale lymfeklier drainage op beeldvorming ontwikkelden thoracale metastasen. Dit zou kunnen duiden op directe interaortocavale drainage naar de longen via de ductus thoracicus en niet via de mediastinale lymfeklieren. De relatief lange ziektevrije overleving moet voorzichtig worden geïnterpreteerd gezien het lage aantal patiënten.

Hoofdstuk 3 rapporteert over een patiënt met cT1bcNOcMO niertumor in een prospectief onderzoek naar schildwachtklieren. De patiënt werd behandeld met een laparoscopische radicale nefrectomie en schildwachtklier bioptie met LND. Na pathologisch onderzoek vond er een upstaging plaats naar een pT1bpN1 papillair II RCC. Twee schildwachtklieren toonden metastasen, maar patiënt bleef ziektevrij na 63 maanden follow-up. Deze case study toont aan dat sommige patiënten met klinisch occulte lymfeklieren lange overleving kunnen hebben na verwijdering van 1 lymfeklier. Identificatie van deze patiënten en lymfeklieren kan uitdagend zijn, terwijl schildwachtklier beeldvorming en biopsie kan helpen bij het bepalen van de eerste drainerende lymfeklieren.

Hoofdstuk 4 evalueerde de topografische verdeling van vroege mono- of oligometasen in lymfeklieren bij nierkanker en heeft vergeleken of deze sites correspondeerden met de anatomische sites van de schildwachtklieren op beeldvorming. We constateerden dat oligometastasen of metastasen in één klier aan de rechterkant voornamelijk hilair, paracavaal, retrocavaal en interaortocavaal liggen en aan de linkerkant in de para-aortale regio. Deze gebieden volgen het patroon van de schildwachtklier studie. De studie wordt beperkt door zijn retrospectieve aard en het ontbreken van een gestandaardiseerd LND-template.

Hoofdstuk 5 analyseerde oorzaken voor de hoge mate van niet-visualisatie met scintigrafie en SPECT/CT in niertumoren. Visualisatie percentage in beeldvorming was $63 \%$ en de enige factor die geassocieerd was met niet-visualisatie was een oudere leeftijd. Met betrekking tot de techniek; de meerderheid van schildwachtklieren werden gevisualiseerd 2-4 uur na de tracer injectie. Zowel de romp als het thoracische gebied moeten worden afgebeeld met planaire scintigrafie in combinatie met SPECT/CT omdat $20 \%$ van de patiënten hun eerst drainerende lymfeklieren in het supradiaphragmatische gebied hadden. Dosering van $225 \mathrm{MBq} 99 \mathrm{mTc}$ was, volgens depot activiteit, optimaal. Vanwege het aantal beperkingen in onze studie zoals post-hoc analyse, laag aantal patiënten, voornamelijk patiënten met een laag risico met een lage incidentie van lymfeklier metastatische RCC, bleef de oorzaak van niet-visualisatie onbekend. 
Hoofdstuk 6 onderzocht de morbiditeit van de schildwachtklier procedure gecombineerd met lymfeklier dissectie en (partiële) nefrectomie. In totaal was het percentage postoperatieve complicaties 16,4\%, waarvan respectievelijk 9,6\% en 6,8\% Clavien Dindo Graad 1-2 en 3a. Er waren geen associaties tussen complicaties en de toegepaste chirurgische modaliteit. Samengenomen kan men zeggen dat de schildwachtklier procedure bij nierkanker veilig is bij zowel open als minimaalinvasieve chirurgie. De analyse is echter gelimiteerd door het ontbreken van een vergelijkende groep en ook door haar post hoc opzet.

\section{Studie perspectieven}

\section{Beeldvorming}

In het tijdperk van immunotherapie bij nierkanker worden prognostificatie technieken en gevalideerde risicomodellen steeds vaker toegepast. Veelgebruikte tools om de invasie van LN te evalueren zijn risicoscores, LND-of beeldvormingstechnieken. Risicoscores die de invasie van lymfeklieren voorspellen, zijn hypothetisch zeer waardevolle hulpmiddelen, maar bij nierkanker is de lage percentage van occulte lymfekliermetastasering het belangrijkste obstakel bij het gebruik van deze in de praktijk [1]. Alle beschikbare nomogrammen om pathologisch lymfeklier metastasen te voorspellen (pN1) gaan alleen omhoog in nauwkeurigheid wanneer lymfeklieren op beeldvorming vergroot zijn (cN1). Daarom is LND nog steeds de gouden standaard en de meest accurate methode voor het opsporen van de invasie van lymfeklieren. Om morbiditeit van LND te voorkomen, wordt de voorkeur gegeven aan minder invasieve methoden.

Schildwachtklier bioptie is een experimentele procedure. Klinische toepassing zou verder onderzoek vereisen bij patiënten met hoog-risico niercelcarcinoom met klinisch kliernegatieve ziekte op beeldvorming ( $\mathrm{CNO}$ ) om het potentieel hogere percentage occulte lymfekliermetastasen in deze setting te bepalen. De dynamische studies van eerste landingsplaatsen hebben ons echter inzicht gegeven in de omvang van lymfedrainage met supradiaphragmatische drainage langs de ductus thoracicus, wat kan helpen verklaren waarom LND voor therapeutisch gebruik tot nu toe geen bewezen overlevingsvoordeel heeft gebracht. Toch heeft de meerderheid van de patiënten schildwachtklieren in de lymfeklieren langs de nierslagaders en aorta aan beide zijden die kunnen worden gebruikt voor prognostische schildwachtklier bioptie-studies bij hoog-risico nierkanker. Dit kan gegevens opleveren over de frequentie van occulte lymfekliermetastase bij nierkanker bij hoogrisico niercelcarcinoom. In het geval van significante metastases kan op die manier de rol van schildwachtklier resectie als therapeutische procedure in verdere prospectieve trials onderzocht worden. Schildwachtklier bioptie, dat nog steeds een invasieve procedure is, moet mogelijk als een experimentele techniek concurreren met opkomende niet-invasieve beeldvormingsmodaliteiten om betrokkenheid van lymfeklieren te detecteren voor stadiëring en prognosebepaling. Conventionele CT-detectie voor lymfeklier metastasen is minder dan $60 \%$, terwijl het kan worden verhoogd met een combinatie van [89Zr] Zr-DFO-girentuximab-PET/CT en [18F]FDG-PET/CT-beeldvorming [2]. In 1 studie met [89Zr] Zr-DFO-girentuximab-PET/CT steeg de detectiepercentage van nierkanker metastasen van $56 \%$ tot $91 \%$. [18F] FDG-PET/CT heeft met respectievelijk $85 \%$ en $>90 \%$ echter een hoger detectiepercentage dan [89Zr] Zr-DFO-girentuximab-PET/CT. Bovendien kan [89Zr] Zr-DFOgirentuximab-PET/CT alleen worden gebruikt in heldercellige niercelcarcinomen, aangezien girentuximab een monoklonaal antilichaam tegen carboanhydrase-9 (CAIX) is, dat tot expressie 
wordt gebracht op deze subtype. Niettemin heeft, gezien het hoge percentage van vals positieve reactieve lymfeklieren in [18F] FDG-PET/CT, [89Zr] Zr-DFO-girentuximab-PET/CT de voorkeur [2]. De beperking van deze studie is het gebrek aan histologische diagnose van lymfekliermetastasen. Girentuximab heeft daarnaast veelbelovende resultaten laten zien in een prospectieve feasibiltystudie in follow-up na cryoablatie [3]. Met andere woorden [89Zr]Zr-DFO-girentuximab-PET/CT is een veelbelovende combinatie van anatomische en functionele beeldvorming die hypothetisch gezien LND ter prognosebepaling in de toekomst zou kunnen vervangen. Verder onderzoek met een groter cohort en evaluatie van de histologie in hoog risico heldercellig niercelcarcinoom is daarvoor vereist. Momenteel is [89Zr]Zr-DFO-girentuximab-PET/CT experimenteel en wordt het onderzocht in een bevestigende internationale trial [NCT03849118].

Schildwachtklier bioptie en haar rol in translationele studies

Een groep uit Scandinavië onderzocht de detectie van afzonderlijke tumorcellen in schildwachtklieren van nierkanker die bij histologisch onderzoek met lichtmicroscopie geen zichtbare metastasen lieten zien [4]. Met kleuring voor de intracellulaire marker cytokeratine 18 (CK18) samen met de oppervlaktemarkers carboanhydrase 9 (CAIX) en Cadherin 6 demonstreerden ze middels flowcytometrie-analyse dat bijna alle schildwachtklieren afzonderlijke tumorcellen bevatten met relatief hoge volumes in vergelijking met conventionele pathologie [4]. Deze observatie moet echter met voorzichtigheid worden geïnterpreteerd. Slechts 15 schildwachtklieren werden geëvalueerd. Deze werden in de lengte doorgesneden en onderzocht, dit in plaats van een schraaptechniek. Dit zou van impact kunnen zijn op het detecteren van micrometastasen gezien de ene helft van de lymfeklier werd onderzocht met immunohistochemie en de andere met H\&E [4]. Desalniettemin suggereert dit potentieel voor translationele onderzoeksprojecten middels schildwachtklier bioptie. Afzonderlijke tumorcellen kunnen worden gesorteerd middels flowcytometrie en vergeleken worden met de genomische eigenschappen van de primaire tumor om een potentiële voorspelling van de lymfatische route te onderzoeken, hierbij wel in gedachte houdend dat nierkanker heterogeen is en het gedrag onvoorspelbaar. Bovendien is het onbekend welke immunogene veranderingen plaatsvinden in de lymfeklieren die de eerste lymfedrainage van de primaire tumor en zijn microomgeving verzorgen. Schildwachtklier bioptie is niet eerder onderzocht bij nierkanker, terwijl het bij andere urogenitale kankers geïmplementeerd is in de behandeling $[5,6,7]$. Naast het belang van de schildwachtklier bioptie voor stadiëring en therapeutische besluitvorming, is de schildwachtklier erkend als de lymfeklier waarin priming van de immuunreactie plaatsvindt. Er zijn gedetailleerde rapporten van onderzoeken uitgevoerd in en ex vivo in immunogene maligniteiten zoals melanomen [8]. Gezien de schildwachtklier de eerste klier is waarop de primaire tumor draineert en dit de voorkeurslocatie is waarnaar de initiële tumor metastateert, wordt deze intensief blootgesteld aan de bioactieve producten van tumorcellen en andere geassocieerde cellen. Dit maakt ze ideaal voor onderzoek van de factoren die de selectieve weefsel gevoeligheid voor metastasen bepalen. In melanomen leidt lokale toediening van GM-CSF en CPG-B oligonucleotiden op de primaire melanoom excisie site tot volledige activering van conventionele dendritische cellen (cDC) en pDC-subsets zowel in schildwachtklieren als in bloed en hun capaciteit suggereert dat hun rekrutering kan bijdragen tot bescherming tegen gemetastaseerde verspreiding door verhoogde cytotoxische T lymfocyten inductie tegen melanoom-afgeleide antigenen [8]. 
Niercelcarcinomen delen veel immunologische kenmerken met melanomen. Onderzoek naar de immuunreactie in de schildwachtklieren is noodzakelijk om te begrijpen of soortgelijke mechanismen van toepassing zijn op niercelcarcinomen. Naar analogie met melanomen zou dit tot nieuwe therapeutische ontwikkelingen kunnen leiden.

We zijn op dit moment van plan om onze prospectieve schildwachtklier bioptie studie te evolueren zodat we de immunologische aard van tumor-drainerende klieren kunnen karakeriseren en een inzicht krijgen in de interactie tussen de componenten van het immuunsysteem en de kwaadaardige cellen. Het belangrijkste doel van het onderzoek is om de kenmerken van de tumor-geassocieerde lymfocyten te beschrijven die betrokken zijn bij anti-tumor reacties in de primaire niertumor, de tumor drainage in de schildwachtklier en elke regionale chirurgisch gesamplede lymfeklier zonder tumor. Dit omvat een verkennende vergelijking van de primaire niertumor met de schildwachtklier en regionale niet-schildwachtklieren, middels immunohistochemie en RNA-sequencing en het correleren van uitkomsten met een potentieel klinische betekenis (recidiefvrije overleving, kankerspecifieke overleving of algehele overleving).

\section{References}

1. Bhindi B, Wallis CJD, Boorjian SA, Thompson RH, Farrell A, Kim, et al. The role of lymph node dissection in the management of renal cell carcinoma: a systematic review and meta- analysis. BJU Int. 2018 May;121(5):684-698.

2. Verhoeff SR, van Es SC, Boon E, van Helden E, Angus L, Elias SG, et al. Lesion detection by [89Zr]ZrDFO-girentuximab and [18F]FDG-PET/CT in patients with newly diagnosed metastatic renal cell carcinoma. Eur J Nucl Med Mol Imaging. 2019;46(9):1931-1939.

3. van Oostenbrugge TJ, Langenhuijsen JF, Oosterwijk E, Boerman OC, Jenniskens SF, Oyen WJG, et al. Follow-up imaging after cryoablation of clear cell renal cell carcinoma is feasible using single photon emission computed tomography with 111In-girentuximab. Eur J Nucl Med Mol Imaging. 2019 Nov 25. doi: 10.1007/s00259-019-04613-z.

4. Hartana CA, Kinn J, Rosenblatt R, Anania S, Alamdari F, Glise H, et al. Detection of micrometastases by flow cytometry in sentinel lymph nodes from patients with renal tumours. $\mathrm{Br} J$ Cancer. 2016:11;115(8):957-966.

5. Sadeghi R, Gholami H, Zakavi SR, Kakhki VR, Tabasi KT, Horenblas S. Accuracy of sentinel lymph node biopsy for inguinal lymph node staging of penile squamous cell carcinoma: systematic review and meta-analysis of the literature. J Urol. 2012;187(1):25-31.

6. Zarifmahmoudi L, Ghorbani H, Sadri K, Tavakkoli M, Keshvari M, Salehi M, et al. Sentinel Node Biopsy in Urothelial Carcinoma of the Bladder: Systematic Review and Meta-Analysis. Urol Int. 2019;103(4):373-382.

7. Wit EMK, Acar C, Grivas N, Yuan C, Horenblas S, Liedberg F, et al. Sentinel Node Procedure in Prostate Cancer: A Systematic Review to Assess Diagnostic Accuracy. Eur Urol. 2017;71(4):596-605.

8. Sluijter BJ, van den Hout MF, Koster BD, van Leeuwen PA, Schneiders FL, van de Ven R, et al. Arming the Melanoma Sentinel Lymph Node through Local Administration of CpG-B and GM-CSF: 
Recruitment and Activation of BDCA3/CD141(+) Dendritic Cells and Enhanced Cross-Presentation. Cancer Immunol Res. 2015;3(5):495-505. 


\section{Acknowledgements}

My deepest gratitude for beyond-phenomenal support and brilliant supervision of Prof Axel Bex who trusted me and shared his prospective study of sentinel lymph nodes in renal tumours and his so valuable and always unique ideas and expertise with me. Thank you for your kind support, patience, motivation, inspiration and comprehensive supervision and honing my scientific, research, clinical and surgical skills. I feel so priviledged of working with such an outstanding and exceptional expert over the years.

I am extremely grateful to Prof Simon Horenblas who made it possible to compile the articles into a thesis and supported my stay at the Netherlands Cancer Institute and who always so kindly conducted MDTs in English and shared his epertise in surgical and scientific level. Thank you for your kind support and inspiration.

My gratitude and acknowledgements for taking their time and critical reading to the reading committee: Prof. Dr. H. P. Beerlage, Prof. Dr. R. De Bree, Prof. Dr. L.M.O de Kort, Prof. Dr. M. G.E.H. Lam, Prof. Dr. Van Leeuwen, Prof. Dr. P.F.A. Mulders and Ceremony Chair Prof. Dr. J.T.A Knape.

I would like to acknowledge my paranymphs for all their kind assistance and friendship Tessa van Ginkel and Mari-Liis Põder.

I would like to express my very deep gratitude for tutorials, collegiality, friendship and support in the Netherlands Cancer Institute: Kees Hendricksen, Bas WG. Van Rhijn, Henk G. Van Der Poel, Esther M. Wit, Pim Van Leeuwen, Oscar R. Brouwer, Niels Graafland, Maarten Donswijk, John Haanen, Jeroen De Jong, Warner Prevoo, Katarzyna Jóźwiak, Renato A. Valdés Olmos and the specialized nurses: Corinne Tillier, Erik van Muilekom, Jolanda Bloos, Eva Offringa and PhD students: Sarah Ottenhof, Charlotte Voskuilen, Joost Blok, Hielke-Martijn de Vries, Elies Fransen van de Putte, co-fellows: Nikolaos Grivas and Mark Behrendt, Tessa Van Ginkel, Rianne Lammers, Roderick De Bruijn and secretaries Marja Van Rijn and Joke Van der Veen. It was so inspiring to work with you all.

I am very grateful to The European Urological Scholarship Programme (EUSP) for financially supporting my stay at the Netherlands Cancer Institute.

I would like to thank for their excellence, speed and high-standard design and technical help Camille Laurelli, Laura Kuusk, Maria Meos and Andre Jagt.

I would like to express my gratitude to all the patients who took part in the trial and all the collaborators and co-authors: Patricia Zondervan, Brunolf Lagerveld, Barak Rosenzweig, Avi Raman, Umberto Capitanio, Andrea Minervini, Borje Ljungberg, Laurence Albiges, Bernard Escudier and Thomas Powles.

I would like to express my deepest gratitude to my Urology residency supervisors: Aare Mehik, Ülo Zirel, Mihhail Zarkovski, Martin Kivi, Andres Kotsar, Markku Onali, Markku Vaarala, Mesrur Selcuk Silay, Fausto Biancari, Tapio Forsell.

Great gratitude to the Royal Free Hospital Renal Unit team: Ravi Barod, Maxine Tran, Faiz Mumtaz, Prasad Patki, Aziz Gulamhusein, David Curry, Pedro, Martha, Angeline, Ryan. I acknowledge greatly your guidance, tutorials and collegiality. 
I am beyond words thankful to my unconditionally loving family: dear mother Anneli Kuusk, dear father Toomas Kuusk, dear sister Laura Kuusk, Camille Laurelli and Vincent Laurelli-Kuusk, dear brother Tõnis Kuusk and Marit Hansen, dear uncle Andrus with his family and dear uncle Tõnu with his family and dearest grandmothers Erna and Hilda and grandfathers IImar and Karl who have always inspired me with their dedicated and creative nature.

My sincerest gratitude to my godmother for her exceptional support, love and encouragements: Airi Põder and godfather Tõnu Põder and my best friend and their daughter Mari-Liis Korjagin-Põder with her family German Korjagin and Georgi Korjagin.

My deep gratitude to my friends whose love and support, even being far, have been accompanying me: Elena and Erich Kõlar, Abdullah Armagan, Veikko Määttä, Madli Haldre, Nelli Roosipuu and Jorge, Jekaterina, Konstantin and Milana Sultanova, Marina Prokopets and Jan, Kärt Kallaste and Annar, Mare Leht and Atte, Ergo Õkva, Tuuli Teeäär and Madis Joonsalu, Anu Salujärv, Irina Sahnjuk, Teele Pern, Maria Meos, Dagmar Meos, Mario Põdra, Stina Tromp, Anne-Liis Kogan, Laura and Tõnis Hallaste, Nathalie Van Zee, Maaike Van Tol, David Miedema, Marc Burrows, Gillian Burrows, Benjamin Smith, Patrick Smith, Louise Hutton, Sunara Begum, Julija Surovecaite, Carolina de Oliveira, Kristjan Haller, Filippos Karageorgiou and his family and Benjamin Goh and his family.

I would like to acknowledge Prof. Bex family for their always warm-hearted support.

\section{Curriculum Vitae}

Dr Teele Kuusk graduated from Medical Faculty of Tartu University in 2009. During her medical studies she was working as sub-investigator at the Clinical Research Center, getting her first experience in clinical vaccine and other medical trials. In 2015 she completed her Urology Residency at Tartu University. During her Urology training, she was working mainly in Oulu University Hospital in Finland and in Tartu University Hospital in Estonia but also had Pediatric Urology training in Bezmialem Hospital in Istanbul supervised by Associate Professor Mesrur Selcuk Silay (European Association of Urology [EAU] Crystal Matula Award 2018, Chair of the Young Academic Urologists (YAU). From 2009-2015 she was representing Estonian Urology residents in the European Society of Residents in Urology (ESRU), organized academic training for Estonian Urology residents and also published her first article: „Treatment of renal angiomyolipoma: pooled analysis of individual patient data." From 2007, she has been attending actively local and international meetings and congresses. After her residency she worked as a Consultant Urologist in Kanta-Häme Central Hospital in Finland. In 2016-2017 she received a prestigeous clinical research fellowship by the European Urological Scholarship Programme (EUSP) at the Center of Excellence, Antoni van Leeuwenhoek HospitalNetherlands Cancer Institute, Amsterdam where she was supervised by renal cancer expert Prof. Dr. Axel Bex. He is a pioneer in using sentinel lymph node technique in renal tumours for the purpose of studying lymphatic drainage, which became Dr Kuusks topic for her thesis. They have won the best poster prize in 2017 for their abstract „Topographic distribution of sentinel lymph nodes in patients with renal tumours" at the Annual Congress of the EAU in 2017 in London. They have published 6 articles on the topic in peer-reviewed Urology journals and have published 14 articles on various 
topics on renal cancer as co-authors. In 2017 Kuusk got selected to attend the Urology masterclass in Salzburg and in 2018 UROBESST course in Berlin. From 2018 she is an associate member of the EAU renal cell carcinoma guidelines panel.

During her career she has given 12 lectures at local meetings, 4 international congresses as an abstract presenter or a session moderator and 2 times been invited speaker at international renal cancer congresses. Also, she is a co-author in the EAU learning module thromboprophylaxy section and Mirrors of Medicine Pinpoint case platform in renal cancer.

In January 2020 she finished her fellowship at the Royal Free London NHS Foundation Trust Renal Cancer Unit where she was trained in robotic, laparoscopic and open surgery in renal cancer.

\section{Publications}

Re: Adjuvant Chemotherapy in Upper Tract Urothelial Carcinoma (the POUT Trial): A Phase 3, Open-label, Randomised Controlled Trial.

Kuusk T, Bex A.

Eur Urol. 2020 Oct;78(4):630-631

Limitations of Available Studies Prevent Reliable Comparison Between Tumour Ablation and Partial Nephrectomy for Patients with Localised Renal Masses: A Systematic Review from the European Association of Urology Renal Cell Cancer Guideline Panel.

Abu-Ghanem Y, Fernández-Pello S, Bex A, Ljungberg B, Albiges L, Dabestani S, Giles RH, Hofmann F, Hora M, Kuczyk MA, Kuusk T, Marconi L, Merseburger AS, Tahbaz R, Staehler M, Volpe A, Powles T, Lam TB, Bensalah K.

Eur Urol Oncol. 2020 Aug;3(4):433-452.

Deferred Cytoreductive Nephrectomy Following Presurgical Vascular Endothelial Growth Factor Receptor-targeted Therapy in Patients with Primary Metastatic Clear Cell Renal Cell Carcinoma: A Pooled Analysis of Prospective Trial Data.

de Bruijn R, Wimalasingham A, Szabados B, Stewart GD, Welsh SJ, Kuusk T, Blank C, Haanen J, Klatte T, Staehler M, Powles T, Bex A.

Eur Urol Oncol. 2020 Jan 16. pii: S2588-9311(20)30001-8. doi: 10.1016/j.euo.2019.12.004. 
Management of Sporadic Renal Angiomyolipomas: A Systematic Review of Available Evidence to Guide Recommendations from the European Association of Urology Renal Cell Carcinoma Guidelines Panel.

Fernández-Pello S, Hora M, Kuusk T, Tahbaz R, Dabestani S, Abu-Ghanem Y, Albiges L, Giles RH, Hofmann F, Kuczyk MA, Lam TB, Marconi L, Merseburger AS, Powles T, Staehler M, Volpe A, Ljungberg B, Bex A, Bensalah K.

Eur Urol Oncol. 2019 Jun 3. pii: S2588-9311(19)30054-9. doi: 10.1016/j.euo.2019.04.005.

Updated European Association of Urology Guidelines on Renal Cell Carcinoma: Immune Checkpoint Inhibition Is the New Backbone in First-line Treatment of Metastatic Clear-cell Renal Cell Carcinoma.

Albiges L, Powles T, Staehler M, Bensalah K, Giles RH, Hora M, Kuczyk MA, Lam TB, Ljungberg B, Marconi L, Merseburger AS, Volpe A, Abu-Ghanem Y, Dabestani S, Fernández-Pello S, Hofmann F, Kuusk T, Tahbaz R, Bex A.

Eur Urol. 2019 May 28. pii: S0302-2838(19)30426-9. doi: 10.1016/j.eururo.2019.05.022.

Cytoreductive nephrectomy in the current treatment algorithm.

Kuusk T, Szabados B, Liu WK, Powles T, Bex A.

Ther Adv Med Oncol. 2019 Sep 27;11:1758835919879026.

Sentinel Lymph Node Biopsy in Renal Tumors: Surgical Technique and Safety.

Kuusk T, Brouwer O, Graafland N, Hendricksen K, Donswijk M, Bex A.

Urology. 2019 Apr 30. pii: S0090-4295(19)30390-5. doi: 10.1016/j.urology.2019.04.026.

A Phase II, single-arm trial of neoadjuvant axitinib plus avelumab in patients with localized renal cell carcinoma who are at high risk of relapse after nephrectomy (NEOAVAX).

Bex A, van Thienen JV, Schrier M, Graafland N, Kuusk T, Hendricksen K, Lagerveld B, Zondervan P, van Moorselaar JA, Blank C, Wilgenbos S, Haanen J.

Future Oncol. 2019 Apr 26. doi: 10.2217/fon-2019-0111.

European Association of Urology Guidelines on Renal Cell Carcinoma: The 2019 Update.

Ljungberg B, Albiges L, Abu-Ghanem Y, Bensalah K, Dabestani S, Fernández-Pello S, Giles RH, Hofmann F, Hora M, Kuczyk MA, Kuusk T, Lam TB, Marconi L, Merseburger AS, Powles T, Staehler M, Tahbaz R, Volpe A, Bex A. 
Eur Urol. 2019 May;75(5):799-810.

Outcome of sentinel lymph node biopsy in patients with clinically non-metastatic renal cell carcinoma.

Kuusk T, De Bruijn R, Brouwer OR, De Jong J, Donswijk M, Hendricksen K, Horenblas S, Jóźwiak K, Prevoo W, Valdés Olmos RA, Van Der Poel HG, Van Rhijn BW, Wit EM, Bex A.

Scand J Urol. 2018 Oct - Dec;52(5-6):411-418.

An analysis of SPECT/CT non-visualization of sentinel lymph nodes in renal tumors.

Kuusk T, Donswijk ML, Valdés Olmos RA, De Bruijn RE, Brouwer OR, Hendricksen $\mathrm{K}$, Horenblas $\mathrm{S}$, Jóźwiak K, Prevoo W, Van Der Poel HG, Van Rhijn BWG, Wit EM, Bex A.

EJNMMI Res. 2018 Dec 3;8(1):105.

Follow-up after curative treatment of localised renal cell carcinoma.

Dabestani S, Marconi L, Kuusk T, Bex A.

World J Urol. 2018 Dec;36(12):1953-1959.

Robotic-assisted Laparoscopic Partial Nephrectomy in a Horseshoe Kidney. A Case Report and Review of the Literature.

Raman A, Kuusk T, Hyde ER, Berger LU, Bex A, Mumtaz F.

Urology. 2018 Apr;114:e3-e5.

Lymphatic Drainage from Renal Tumors in Vivo: A Prospective Sentinel Node Study Using SPECT/CT imaging.

Kuusk T, De Bruijn R, Brouwer OR, De Jong J, Donswijk M, Grivas N, Hendricksen K, Horenblas S, Prevoo W, Valdés Olmos RA, Van Der Poel HG, Van Rhijn BW, Wit EM, Bex A.

J Urol. 2017 Dec 6. doi: 10.1016/j.juro.2017.11.112.

Observation After Cytoreductive Nephrectomy in Patients With Synchronous Not Completely Resected Metastases of Renal Cell Carcinoma.

de Bruijn RE, Kuusk T, Noe AP, Blank CU, Haanen JBAG, Hendricksen K, Horenblas S, Bex A.

Urology. 2017 Nov;109:127-133. 
The impact of adding sentinel node biopsy to extended pelvic lymph node dissection on the biochemical recurrence of prostate cancer patients treated with robot-assisted radical prostatectomy.

Grivas N, Wit E, Kuusk T, KleinJan G, Donswijk M, van Leeuwen F, van der Poel H.

J Nucl Med. 2018 Feb;59(2):204-209.

External beam radiation for the treatment of castration-resistant prostate cancer following primary hormonal therapy with androgen ablation: Analysis and outcome of 21 patients.

Kuusk T, Pulliainen $\mathrm{K}$, Vaarala $\mathrm{MH}$.

Mol Clin Oncol. 2017 Mar;6(3):428-432.

Antiangiogenic therapy combined with immune checkpoint blockade in renal cancer.

Kuusk T, Albiges L, Escudier B, Grivas N, Haanen J, Powles T, Bex A.

Angiogenesis. 2017 May;20(2):205-215.

Long-term Survival After Resection of Sentinel Node Metastatic Renal Cell Carcinoma.

Kuusk T, De Jong J, Grivas N, Horenblas S, Bex A.

Urology. 2017 May;103:e5-e6.

The current management of renal cell carcinoma.

Kuusk T, Grivas N, de Bruijn R, Bex A.

Minerva Med. 2017 Aug;108(4):357-369.

Re: Detection of Micrometastases by Flow Cytometry in Sentinel Lymph tients with Renal Tumours. Kuusk T, Bex A.

Eur Urol. 2017 Apr;71(4):691-692.

Treatment of renal angiomyolipoma: pooled analysis of individual patient data.

Kuusk T, Biancari F, Lane B, Tobert C, Campbell S, Rimon U, D'Andrea V, Mehik A, Vaarala MH. BMC Urol.2015 Dec 28;15:123. 
EUSP Fellowship Report; Innovative research environment at the Netherlands Cancer Institute 12/2017 European Urology Today

Paediatric urology training in Istanbul 4/2015;24 European Urology Today

Young urology resident looks back at 25th Anniversary EAU Congress 3/2010; 15 European Urology Today 